\title{
Synaptopodin is required for stress fiber and contractomere assembly at the epithelial junction
}

\author{
Timothy Morris, Eva Sue, Caleb Geniesse, William M Brieher, and Vivian W Tang* \\ Department of Cell and Developmental Biology, University of Illinois, Urbana-Champaign \\ ${ }^{*}$ Corresponding author: vtang@illinois.edu
}

\begin{abstract}
The apical junction of epithelial cells can generate force to control cell geometry and perform contractile processes while maintaining barrier function and cell-cell adhesion. Yet, the structural basis of force generation at the apical junction is not completely understood. Here, we describe 2 actomyosin structures at the apical junction containing synaptopodin, myosin IIB, and alpha-actinin-4. We showed that synaptopodin is required for the assembly of E-cadherin-associated apical stress fibers and a novel macromolecular structure, which we named contractomere. Knockdown of synaptopodin abolished both apical stress fiber and contractomere formation. Moreover, depletion of synaptopodin abolished basal stress fibers, converting myosin IIA sarcomere-like arrangement into a meshwork-type actomyosin organization. We propose a new model of junction dynamics that is dependent on contractomere movement to control epithelial cell boundary and geometry. Our findings reveal 2 actomyosin structures at the epithelial junction and underscore synaptopodin in the assembly of stress fibers and contractomeres.
\end{abstract}

Short Running Title: Stress fiber and contractomere require synaptopodin

Summary Statement: Synaptopodin assembles 2 actomyosin structures at the epithelial junction: apical stress fiber and contractomere. Synaptopodin selectively regulates myosin IIB without altering the level of myosin IIA and is responsible for converting evolutionaryconserved actomyosin meshwork into vertebrate-specific stress fibers. 


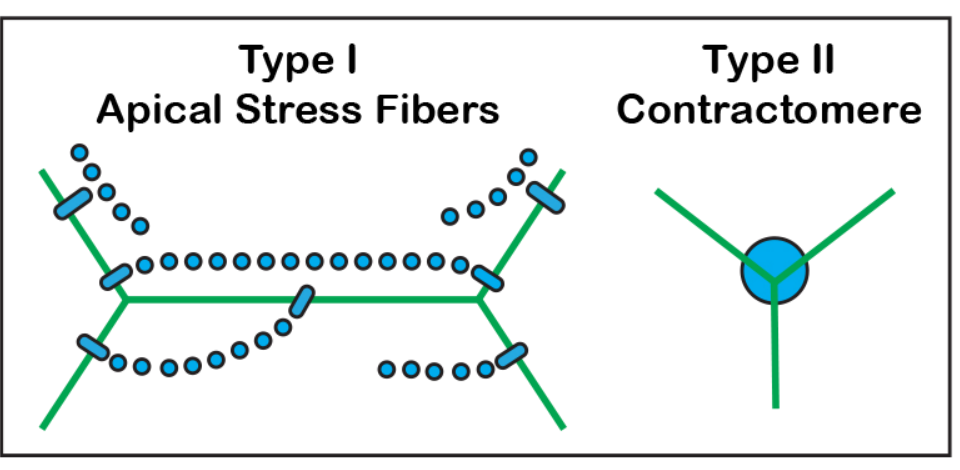

Side-on
Apical Stress Fibers

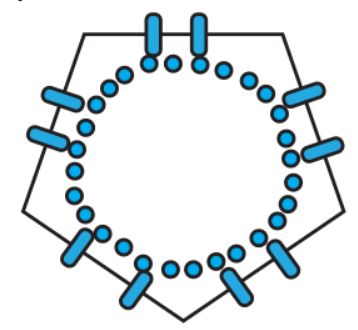

Force generated far away from Junction

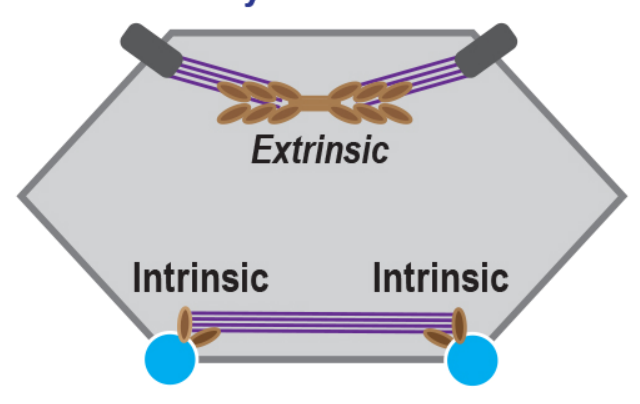

Force generated directly by the Junction
Multicellular Force Transmission via Head-on Apical Stress Fibers

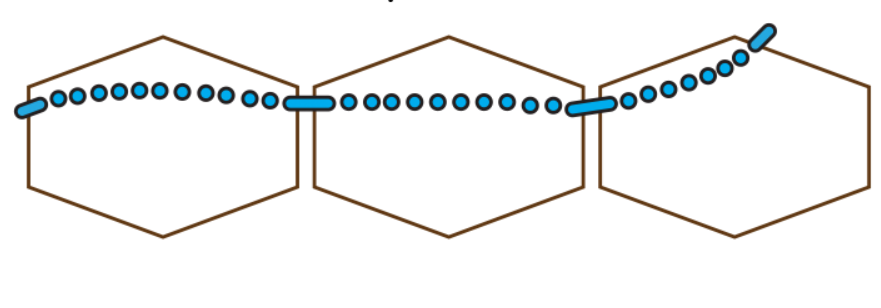

Compressive Force exerted on junctional a-actinin-actin cortex

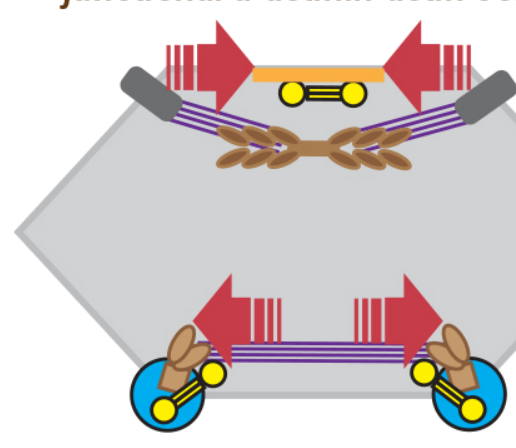

Compressive Force exerted on contractomeric a-actinin-actin complex 


\section{Introduction}

Epithelial cells cover body cavities and line internal organs, providing continuous protection from biological, chemical, and mechanical insults. The maintenance, stability, and physiological functions of an epithelium require the assembly of a specialized apical junction. The apical junction provides adhesions between cells, forms paracellular barrier, and generates force to regulate cell geometry. Force exerted by the epithelial junction not only can reshape cell boundaries and adjust junctional length but also facilitate cell rearrangement during dynamics processes including wound migration and morphogenesis (Pinheiro and Bellaiche, 2018). Moreover, junction contractility is necessary for cell extrusion and purse-string wound closure that are essential for epithelial homeostasis (Bement et al., 1993; Clark et al., 2009; Danjo and Gipson, 1998; Florian et al., 2002).

Force generation at the junction depends on myosin II, a $>300 \mathrm{~nm}$ bipolar minifilament consisted of anti-parallel arrangement of barbed-end-directed myosin II motors. Activation of junctional contractility results in shortening of the junction and destabilizes E-cadherin adhesions (Cavanaugh et al., 2020). In contrast, application of orthogonal force to the junction results in strengthening of E-cadherin adhesions (Gomez et al., 2011; Kannan and Tang, 2015; Kannan and Tang, 2018; le Duc et al., 2010). Thus, the biological outcome of force at the junction appears to depend on the orientation of the applied force, which is the directional information embedded within the force vector.

What is the molecular and structural basis that allows the generation of orthogonal and parallel force at the apical junction? How does the cell organize actomyosin populations to control the direction of force? Can the junction pull on actin filaments or is the junction being pulled by actomyosin? Do we know whether contractile force is generated at the junction or is the junction on the receiving end of force? At least 2 actomyosin structures could exert orthogonal force on the apical junction. The apical-medial actomyosin meshwork in polarized epithelial cells can generate isotropic contractile force, pulling the junction orthogonally (Roper, 2015). Contraction of cytoplasmic actomyosin network also can exert force on cell-cell adhesions (Kannan and Tang, 2015; Wu et al., 2014). Myosin IIA is organized into actin cables underneath the junction whereas myosin IIB is found on membrane adhesions. Contractions of these 2 actomyosin populations could exert parallel force on the junction (Heuze et al., 2019). Earlier studies show that myosin IIB controls actin accumulation whereas myosin IIA regulates E-cadherin stability (Smutny et al., 2010). Furthermore, myosin IIB, but not myosin IIA, plays a role in alpha-catenin mechanoregulation (Heuze et al., 2019). Thus, different myosin II isoforms can form actomyosin structures to serve distinct force-dependent functions.

In this paper, we describe 2 actomyosin structures, apical stress fiber and contractomere. We provide evidence for an essential molecular component for their assembly and propose a new mechanism for junction remodeling to control cell geometry during apical junction constriction and intercellular movement. Our results provide insight into the structural basis of force production and transmission, underscoring the complexity of mechanoregulation at the epithelial apical junction. 


\section{Results}

To understand how actomyosin organization contributes to force generation at the epithelial junction, we focused on 3 actin-binding proteins, alpha-actinin-4, synaptopodin, and myosin IIB, which are known regulators of cellular contractility. Synaptopodin is a vertebrate-specific protein expressed ubiquitously in human (Uhlen et al., 2015). Using antibodies against different spliced regions of synaptopodin, we showed that MDCK cells primarily express synaptopodin isoform A (Fig S1A-B). In this study, synaptopodin A is referred to as synaptopodin since it is the predominant form in MDCK cells.

\section{Epithelial junction contains 2 populations of myosin IIB}

MDCK cell monolayers grown on Transwells develop apical junctions that have strong cellcell adhesion and a robust permeability barrier (Kannan and Tang, 2015; Tang and Goodenough, 2003). We grew MDCK cells on Transwells and compared their actomyosin structures at various stages of junction development using superresolution immunofluorescence microscopy. At an early stage of junction development, myosin IIB and synaptopodin exhibit periodic and alternating pattern of arrangement (Fig $1 \mathrm{~A})$. Alternating pattern of myosin II and alpha-actinin is a signature organization for contractile basal stress fibers, often referred to as sarcomere-like (Naumanen et al., 2008; Pellegrin and Mellor, 2007). Consistent with previous biochemical data that shows direct interaction between synaptopodin and alpha-actinin-4 (Asanuma et al., 2005; Kremerskothen et al., 2005), they overlap strongly on apical stress fibers (Fig 1B \& insets). On the basal surfaces of the monolayer, stress fibers are characterized by periodic and alternating pattern of myosin IIB and synaptopodin (Fig S2A). Synaptopodin-containing basal stress fibers insert at their ends into vinculin-decorated focal adhesions (Fig S2B). Both apical and basal stress fibers have myosin IIA periodic arrangements (Fig S3A-B).

MDCK monolayers gradually develop mature junctions over 2-3 weeks, which coincide with the disappearance of apical stress fibers (Fig 1C). During this transitional period, a new structure containing myosin IIB and synaptopodin is formed (Fig 1C, blue box). Residual apical stress fibers with alternating myosin IIB and synaptopodin can be found next to the new structure (Fig $1 \mathrm{C}$, red box). The disassembly of apical stress fibers is restricted to the junction since basal stress fibers remain present (Fig $1 \mathrm{C}$, yellow box). We refer to these 2 different myosin IIB structures as "type I" when myosin IIB and synaptopodin are arranged in alternating pattern and "type II" when they overlap.

\section{Apical stress fiber is inserted at E-cadherin junction}

To assess the relationship between cell-cell adhesion and myosin IIB structures, we performed superresolution immunofluorescence microscopy for E-cadherin, myosin IIB and synaptopodin. We found that apical stress fibers are positioned parallel to the junction and spatially separated from E-cadherin junctions except at points of insertion (Fig 2A-B). 
This is analogous to basal stress fibers where their attachments at focal adhesions occur at the ends of the stress fibers. In the course of junction development, apical stress fibers form side-on interactions at synaptopodin-decorated sites with along the length of Ecadherin junctions (Fig 2A-B, arrowheads). Upon association with E-cadherin junctions, apical stress fibers become less organized and the characteristic sarcomeric-repeats are less recognizable. As junctions mature over time, the alternating pattern of myosin IIB and synaptopodin completely dissolves (Fig 2C, left and middle panels). Concomitantly, Ecadherin, myosin IIB, and synaptopodin accumulate at the ends of linear junctions, which coincide frequently with the v-junctions, also known as 3-way junctions or vertices (Fig 2C, graph). Upon maturation of the epithelial monolayer, apical stress fibers are absent and the junctions now contain primarily type II myosin IIB structures enriched in alpha-actinin-4, synaptopodin, and actin (Fig 2D-E).

\section{Synaptopodin is a marker for apical stress fiber}

Synaptopodin emerges as one of the best markers for cytoskeletal structures. To watch synaptopodin dynamics, we expressed synaptopodin tagged with venus fluorescent protein in MDCK cells. We found that synaptopodin-venus is organized in periodic intervals, decorating both apical and basal stress fibers in live cells (Fig 3A-B). Live-cell imaging of synaptopodin revealed that synaptopodin apical stress fibers are either inserted head-on or side-on to the junction (Fig 3A-B). These 2 configurations have important implications in force transmission at the apical junction. If apical stress fiber is attached head-on, the junction would experience orthogonal force during contraction of the apical stress fiber. Head-on attachment, in principle, would allow the cell to sense stiffness of the junction and provide maximally resistance to external stretching force. By contrast, sideon interactions wound exert parallel force to the junction, facilitating lateral movement and clustering of adhesion complexes. Contraction of apical stress fibers could specifically exert force at sites of insertion to increase tension locally at cell-cell adhesions.

Live-cell imaging also showed that apical stress fibers can be arranged to form a circular structure that has no end, roughly tracing the cell boundary (Fig. 3B, left panel). In this organization, apical stress fibers are linked via head-on as well as side-on attachments to the junctions. Centripetally contraction of circular apical stress fiber would exert orthogonal force on the apical junction. Time-lapse movie of synaptopodin showed that apical stress fibers are stable structures and would float inside the cytoplasm during contractions (Movie 1). The 2 configurations of "type I" actomyosin IIB organization are likely to play distinct roles at the junction; thus, we classify them into "type la" for head-on attachment and "type lb" for side-on attachment (Fig 3A).

\section{Synaptopodin marks myosin IIB-containing junctional complex}

Live-imaging of synaptopodin showed that the periodic pattern of synaptopodin in apical stress fiber transitions to a diffuse localization during junction maturation (Fig $3 \mathrm{C}$, left panel). The dissolution of apical stress fibers is specific to the apical junction because stress fibers at the basal surface remain present (Fig 3C, right panel). These observations 
implicated a mechanism at the junction that is capable of locally and specifically disassembling apical stress fibers. Concomitantly, synaptopodin was found to accumulate at the junctional poles (Fig 3C, middle panel), corresponding to type II myosin IIB structure (Fig 1C \& 2D-E). Since myosin IIB and synaptopodin type II structure has not been previously described prior to this study, we named it "contractomere"; "contracto" refers to the fact that it is a contractile structure and "mere" refers to the fact that it is the minimal macromolecular complex for its contractile activity.

\section{Junction localization of myosin IIB requires synaptopodin}

To determine the role of synaptopodin, we used our previously established synaptopodin knockdown cells (Kannan and Tang, 2015). We found that, when synaptopodin was depleted, myosin IIB structures were absent from the junction (Fig. 4A-B). To assess the dependency of myosin IIB on synaptopodin, we measured their levels at the junction and found that they are tightly correlated (Fig 4C). On the basal surface, stress fibers were also missing in synaptopodin knockdown cells (Fig 4D). Yet, the depletion of synaptopodin did not result in overt changes in cell morphology (Fig S4), and junctional actin was not grossly affected (Fig S5). Myosin IIA remains present at the apical junction (Fig S6A). Nevertheless, on the basal surface, myosin IIA is now converted from stress fibers to a meshwork-type organization (Fig S6B). Depletion of synaptopodin completely blocked myosin IIB and alpha-actinin-4 accumulation at v-junctions (Fig 4E-G). Western blots of total cell lysate showed that myosin IIB and phospho-myosin light chain levels were downregulated in synaptopodin knockdown cells (Fig $4 \mathrm{H})$. These results demonstrate that synaptopodin is essential for the assembly of myosin IIB-dependent structures including stress fibers and contractomeres.

\section{Knockdown of synaptopodin disrupts junction tension balance}

One important function of junction contractility is to constrict the apical junction during wound healing and cell extrusion. It has been established that cell extrusion can be triggered mechanically by contraction of the dying cell (Kuipers et al., 2014), indicating that exerting force on the junction could activate contractility of neighboring cell junctions. Thus, cell extrusion can be viewed as a process that is controlled by junctional tension and the imbalance of junctional tension can lead to cell extrusion (Eisenhoffer et al., 2012; Franco et al., 2019; Nanavati et al., 2020; Saw et al., 2017). To determine the role of synaptopodin-dependent structures in the regulation of junctional tension, we asked if synaptopodin knockdown affects tension-induced cell extrusion (Fig 5A). We used our previously established hydraulic apparatus to activate junction contraction and monitored tension-activated cell extrusion (Fig 5B). We found that, in the absence of synaptopodindependent actomyosin structures, application of force to the junction resulted in aberrant cell extrusion (Fig 5C). Our result is consistent with the idea that balancing junctional force is essential for epithelial homeostasis, and synaptopodin plays an important role in this process. 
From the results we presented so far, it is clear that there are at least 2 distinct synaptopodin-dependent myosin IIB structures at the apical junction. Therefore, knockdown of synaptopodin will not be useful for distinguishing the independent contributions of these 2 structures. We will have to either develop new biochemical techniques or cellular assays to study their functions. For the remainder of this study, we will focus on the contractomere since it is a novel structure that is almost certainly a vertebrate invention.

\section{Blebbistatin blocks actin accumulation at the contractomere}

The goal of the remainder of this study is to address whether the contractomere is a new organelle possessing unique activities that can serve novel functions at the apical junction.

Previously, we reported that treatment of mature cell monolayer with latrunculin B, an actin monomer sequestration drug that promotes actin dissociation from filament ends, resulted in the depolymerization of almost all cellular actin structures except a latrunculin-resistant pool associated with alpha-actinin-4 at the apical junction (Tang and Brieher, 2012). We now realize that these alpha-actinin-4-enriched latrunculin-resistant structures are contractomeres. We are very intrigued by the enrichment of myosin IIB at these sites and suspect that myosin ATPase activity plays a role in actin accumulation. To develop this idea further, we assessed junctional actin using a combination of well-characterized inhibitors of actin dynamics. We found that blebbistatin, a myosin II ATPase inhibitor (Limouze et al., 2004; Ramamurthy et al., 2004), completely prevented the formation of latrunculin-resistant actin (Fig 6A) which are usually marked by synaptopodin (Fig 6B).

How does the contractomere accumulate actin in the presence of latrunculin B, which creates an environment that favors depolymerization of actin filaments? One possibility is that the contractomere can polymerize actin using latrunculin-bound actin. To eliminate this highly unlikely possibility, we blocked barbed-end dynamics using cytochalasin D to prevent actin polymerization (Fig 6C, left bottom 2 rows). Strikingly, barbed-end capping actually promotes actin accumulation at the contractomere under depolymerization environment. One interpretation of this intriguing result is that the motility function of myosin IIB at the contractomere is capable of possessively "walk" on actin filament and "wheel-in" actin filaments to form an actin ball. If this is true, blocking myosin II activity should prevent actin accumulation. Indeed, blebbistatin treatment completely abolished latrunculin-induced actin accumulation at the contractomere, either in the presence or absence of cytochalasin D (Fig 6C, second and fourth row). These observations support our hypothesis that contractomeric myosin IIB possessively "walk" on actin filaments, conferring an actin motility function to the junctional complex.

We further explored the relationship between actin motility and junction contractility by repeating the drug experiments on small wounds (Fig 6D). Treatment with latrunculin $B$ resulted in the formation of latrunculin-resistant actin puncta around the wound edge (Fig 6D, middle 2 panels in top row) which formation is inhibited by blebbistatin (Fig 6D, left panel in top row). In control cells, actin-decorated puncta appears to relocation during 
wound closure (Fig 6D, right panels). This interesting observation suggests that the contractomeres might move during wound constriction.

\section{Contractomere couples myosin activity and actin polymerization}

Contractomere was originally identified in a junction-enriched membrane fraction containing de novo actin assembly activity (Tang and Brieher, 2012). This activity requires Arp2/3-dependent actin nucleation and alpha-actinin-4-dependent actin polymerization. At the apical junction of polarized epithelial cells, a-actinin-4-dependent actin assembly occurs at sites marked by latrunculin-resistant actin. Using chemical crosslinking of alphaactinin-4, we identified synaptopodin in ex vivo junction-enriched membranes (Tang and Brieher, 2013). Subsequently, we showed that synaptopodin interacts with myosin II and forms a complex with beta-catenin and alpha-actinin-4 in cell lysates (Kannan and Tang, 2015). These data, together with our current results, implicate a role of myosin II in contractomeric actin assembly.

Myosin IIB is highly enriched in contractomeres in cells and in ex vivo junction-enriched membranes (Fig 7A-B). To determine whether actin assembly in vitro requires myosin II ATPase activity, we performed actin assembly assays using junction-enriched membranes (Fig. 7C). In an actin assembly assay, actin assembly is initiated by the addition of 2 uM of fluorescently-labelled monomeric actin, a concentration substantially above the critical concentration of actin, to junction-enriched membranes in the presence of ATP. Timelapse movie showed that actin polymerization is concomitant with contraction of junctional membranes (Movie 2). Both actin assembly and membrane contraction were blocked by blebbistatin (Movie 3). Immunofluorescence of junction-enriched membranes showed that myosin IIB is enriched at sites of actin assembly, indicating that contraction originates at the contractomeres and is coupled to actin polymerization (Fig 7D \& S7). Collectively, these findings revealed that that contractomeres have constitutively active myosin II ATPase activity and could potentially power actin motility to support junction contractility. Our results are consistent with previous laser ablation experiments demonstrating that junction contraction requires both actin polymerization and myosin II ATPase activity (Arnold et al., 2019; Verma et al., 2012).

A myosin II minifilament is $\sim 300 \mathrm{~nm}$ in length characterized by a dumbbell-shape with myosin heads spraying out at both ends and a bare mid-zone (Liu et al., 2017; Liu et al., 2018). Yet, we had never seen any structure resembling a myosin II minifilament in junction-enriched membranes in negative-stain electron microscopy (EM). Purified contractomere is $<250 \mathrm{~nm}$ in dimension (Kannan and Tang, 2015), which is too small to hold a myosin II minifilament. In fact, extraction of junctional membranes with non-ionic detergent revealed a contractomere no bigger than $150 \mathrm{~nm}$ in dimension but still can interact with actin filaments at multiple sites (Kannan and Tang, 2015). Recently, monomeric myosin II has emerged as an important player in diverse cellular functions including focal adhesion initiation, cell migration, Golgi dynamics, and exocytosis (Aoki et al., 2010; Ganguly et al., 1992; Kiboku et al., 2013; Shutova et al., 2017; Shutova et al., 2014). Here, we used a modified protocol to investigate whether contractomere might 
contain monomeric myosin II (Fig 7E). We empirically tested extraction conditions by varying the concentrations of different chaotropic agents and then screened for conditions that might break the contractomere into subcomplexes. We found one condition using mechanical shearing and sequential extractions with low salt followed by high pH buffers; the dissociated subcomplex contains a myosin monomer interacting with an actin filament (Fig. 7E, right panel, yellow arrows). In negative-stain EM, the myosin monomer is bound to electron dense materials at its coiled-coil tail (Fig. 7E, right panel, red arrows). These observations are consistent with recently published works by the Svitkina group showing electron-dense "stuff" bound to the coiled-coil tail of monomeric myosin II in cells (Shutova et al., 2017; Shutova et al., 2014).

Our data revealed that the contractomere interacts with single actin filaments. Yet, contractomeric actin assembly requires alpha-actinin-4, an actin crosslinking protein. Why wound actin assembly require an actin crosslinking protein? Why can't we find any actin networks if there were actin cross-linking? One possibility is that alpha-actinin-4 does not function as an actin crosslinker at the contractomere. To determine if crosslinking is necessary for contractomeric actin assembly, we performed a reconstitution assay where the membranes were stripped with high salt to remove endogenous alpha-actinin-4 and replaced with recombinant alpha-actinin-4 (Tang and Brieher, 2012; Tang and Brieher, 2013). Using the reconstitution assay, we can test different truncations of alpha-actinin-4 to determine whether actin crosslinking is necessary for actin assembly.

Alpha-actinin exists as an anti-parallel dimer $\sim 36 \mathrm{~nm}$ in length (Meyer and Aebi, 1990) with 2 actin-binding domains flanked by spectrin repeats that interact heterotypically (Liu et al., 2004). We generated recombinant alpha-actinin-4 that has 1 actin-binding domain instead of 2 actin-binding domains, thus cannot crosslink actin filaments into networks. For comparison, we generated alpha-actinin-4 that has no actin-binding domain or missing the spectrin repeats (Fig 7F). After we obtained recombinant alpha-actinin-4 proteins, we labelled them with a fluorophore so that we can track their targeting to the contractomere (Fig 7G). We found that actin-binding is not required for alpha-actinin-4 recruitment; alphaactinin-4 missing both actin-binding domains can still target to the contractomere (Fig 7G). This is in sharp contrast to other actomyosin structures such as stress fiber or meshwork where actin-binding is integral to their assembly. Moreover, alpha-actinin-4 with only 1 actin-binding domain would support actin assembly to the same extend as alpha-actinin-4 with 2 actin-binding domains (Fig $7 \mathrm{H})$. However, spectrin repeats alone or actin-binding domain alone did not support actin assembly, indicating that alpha-actinin-4 targeting and actin-binding are both necessary for contractomeric actin assembly (Fig $7 \mathrm{H})$. These results demonstrated that the contractomere exhibits novel and unique biochemistry distinct from any known alpha-actinin functions or myosin II structures.

\section{Contractomere moves to shorten or lengthen a junction}

The unique biochemical activities of the contractomere provide an opportunity for the cells to invent novel junction processes and perhaps new epithelial behaviors. Myosin IIB is a vertebrate paralog that has higher duty ratio and produces greater power than the 
ancestrally-derived myosin IIA (Melli et al., 2018; Stam et al., 2015; Wang et al., 2003). Therefore, incorporating myosin IIB not only confers "motor" function on the contractomere but allows possessive movement of the contractomere on actin filaments. To assess whether contractomeres can "walk the junction", we performed live-cell imaging using venus-alpha-actinin or venus-synaptopodin as contractomere markers. Live-cell imaging of alpha-actinin showed that contractomeres surrounding the extruding cell move towards each other during apoptotic cell extrusion (Fig. 8A \& Movie 4). As a consequence, the distance between individual contractomere shortens, leading to constriction of the junctions surrounding the extruding cell.

Contractomere motility is also associated with live-cell extrusion in the absence of apoptotic blebbing (Fig. 8B \& Movie 5). Again, the contractomeres moved towards each other during junction shortening. At the end of junction constriction, the contractomeres became very close to each other and their fluorescent signals were overwhelmed by outof-focus light from the extruding cell. To visualize discreet contractomeres without the interference by out-of-focus light, we imaged cell extrusion using structured-illumination live-cell microscopy (Movie 6-7). Now, we could track the trajectories of individual contractomeres all the way to the end of a constriction event (Fig 8C-D, circles). Movement of contractomeres towards each other effectively zipped-up the junctions, preserving the epithelial barrier during cell extrusion.

Tracking the trajectories of moving contractomeres during cell extrusion (Movie 8) revealed that shortening of a junction (Fig 8E, yellow and green arrows) is associated with concomitant elongation of neighboring junctions, resulting in the formation of a new junction between adjoining cells (Fig 8E, blue and orange arrows). Thus, the motility of contractomeres can separate 2 cells by un-zipping the junction and zip-up neighboring cells to form new junction. These observations suggest that the contractomere can change the proportions of junctional lengths in a cell by simply moving around. This mechanism of junction remodeling is distinct from the recycling model where existing junction is resorbed via endocytosis (Cavanaugh et al., 2020).

The ability to change the length proportions of junctions in a cell provides a new mechanism for cell rearrangement in an epithelial cell monolayer. To explore this idea further, we performed live-cell imaging using structured-illumination live-cell microscopy. Analysis of junctional lengths indeed showed shortening of some junctions and extension of other junctions during intercellular movement (Fig 8F \& S8A, Movie 9). Junctional length shortens when 2 contractomeres move towards each other and extends when 2 contractomeres move away from each other (Fig 8F). Our hypothesis also requires that the sum of junctional lengths of an individual cell stays relatively constant during cell rearrangement, which was what we observed (Fig S8B \& Movie 10). In mature cell monolayers, contractomeres continue to oscillate at a smaller length-scale and a faster time-scale (Fig 8G \& H, Movies 11-15), a behavior that we had previously reported (Kannan and Tang, 2015). Our observations are consistent with the molecular properties of myosin IIB motor, which has the ability to step forward and backward in response to load, allowing myosin IIB to oscillating and generate tension on actin filaments (Norstrom 
et al., 2010). Our data is consistent with live-imaging of actin biosensors in Xenopus mesoderm showing that pulsed actin assembly at v-junctions is associated with shortening of the linear junction (Shindo and Wallingford, 2014). Our discovery and characterization of the contractomere underscores a novel mechanism for junction dynamics and epithelial cell behavior.

Our findings indicate that the proportion of junctional lengths, the overall shape of the cell, and the position of the cell within a monolayer can be adjusted quickly by sliding the contractomeres around the boundary of epithelial cells embedded within a confluent cell monolayer. Moreover, in steady-state, contractomeres could contribute to the generation of junctional tension provided by the motility function of myosin IIB.

\section{Compressive force is experienced by alpha-actinin-4 at the contractomere}

To investigate the molecular forces experienced by alpha-actinin at the junction, we tested 4 different alpha-actinin tension sensors (Fig 9A). Two of the tension sensors were designed by the Sachs group in which a FRET-based tension cassette, sstFRET, was inserted into 2 different regions of alpha-actinin-1 (Meng and Sachs, 2012). The tension cassette, sstFRET, contains a single spectrin repeat flanked by a venus-cerulean FRETpair, which reports on $\sim 6 \mathrm{pN}$ of force with $\sim 50 \%$ change in FRET (Meng and Sachs, 2011). We had previously used the sstFRET cassette to measure E-cadherin and myosin-1c tension in epithelial cell sheets, indicating that the sensor is capable of reporting on molecular forces at the junction (Kannan and Tang, 2018). We found that alpha-actinin-1 that has sstFRET linked to its C-terminus (a-actinin-1-sstFRET-C), which presumably experiences no externally applied force, exhibited a FRET index of $\sim 0.65$ in live cells (Fig. 9A). Previous studies had used an alpha-actinin-1 with sstFRET inserted within the first spectrin repeat to measure forces in stress fibers (Verma et al., 2015). Using this sensor, we found that the junction is under tensile force (Fig 9A, a-actinin-1-M-sstFRET). Since alpha-actinin-4 is the predominant alpha-actinin in MDCK cell, we designed 2 additional tension sensors by positioning the sstFRET cassette at locations that we think might report on changes in alpha-actinin-4 tension (Fig. 9A). We inserted the sstFRET cassette between the 1st and 2nd spectrin repeats (a-actinin-4-sstFRET408) or between the 2nd and 3rd spectrin repeats (a-actinin-4-sstFRET522) of human alpha-actinin-4. To our surprise, both alpha-actinin-4 intramolecular tension sensors experienced compressive force, rather than tensile force (Fig 9A).

To determine which of the alpha-actinin tension sensors is reporting forces that are applied to alpha-actinin, we compared FRET in protrusive and tensile structures (Fig 10B-D). We found that a-actinin-1-sstFRET-C and a-actinin-1-M-sstFRET were unable to distinguish forces at cell protrusion and cell junction (Fig 10B-C). By contrast, a-actinin-4-sstFRET522 showed dramatic FRET differences between protrusions at free cell edge and cell junction (Fig 9D). The FRET index of a-actinin-4-sstFRET522 at cell protrusions was $\sim 0.62-0.65$, similar to the FRET index measured using a-actinin-1-sstFRET-C, indicating that a-actinin4-sstFRET522 was not experiencing externally applied force at the cell edge. However, at cell junction, the FRET index increased almost 0.2 corresponding to a few $\mathrm{pN}$ of force. 
Therefore, out of the 4 constructs, only a-actinin-4-sstFRET522 was useful as an intramolecular tension sensor.

To determine whether contractomeres are under tension, FRET indexes were measured at multiple locations including cell protrusions, cytoplasm, linear junctions, and contractomere (Fig. 9E). To our surprise again, we found a dramatic increase in compressive force at the contractomere, at a level that is even higher than the FRET index measured on the linear junction (Fig. 9E). Our findings are opposite of what we would expect because myosin II contractility has always been thought to pull on the junction, which should register as tensile force rather than compressive force. Yet, these observations are consistent with our biochemistry results. If myosin IIB is pulling on actin filaments towards the contractomere, it would shove actin towards alpha-actinin 4 in the complex, exerting a compressive force on alpha-actinin-4 located within the contractomere (Fig. 9F \& S9 for symbol legend). Our measurements of compressive force on the linear junction implies at least 3 possibilities. One possibility is that the intracellular environment of epithelial cell monolayer is under compression. The second possibility is that compression is local and is produced by contraction of apical stress fiber or contractomere (Fig. 9F). A third possibility is that actin protrusions push on the junction, pressing the adhesion cortex ( $\mathrm{Li}$ et al., 2020). Thus, the current paradigm of junctional forces should to be rewritten, at least for vertebrate epithelial cells.

\section{Re-defining actomyosin structures at the epithelial junction}

Fig 10 presents a summary of actomyosin structures at the epithelial junction. The prevailing models describe 3 different actomyosin organizations for force generation, all of them depend on bipolar myosin II minifilaments (Fig 10A-B). The first model describes a sub-membrane an actomyosin network (Fig 10A, upper left). The second model describes actin bundles decorated with myosin II (Fig 10A, upper right). The third model describes an isotropic actomyosin II meshwork on the apical-medial cortex (Fig 10B, upper left). These 3 structures are likely to exert orthogonal force on the junction. However, they fall short in explaining how the junction shrink during wound constriction, cell extrusion, or intercellular rearrangements. The 2 major issues are as follows.

The first issue is the fluid mosaic problem. In the first and second models stated above, force is exerted parallel to the junction, pulling adhesion complexes on the plane of the membrane. Parallel force would move adhesion complexes on the plane of the plasma membrane and potentially causing them to cluster. This mechanism will leave the lipids of the plasma membrane untouched rather than shrinkage the junctional membrane. In the third model stated above, contraction of apical isotropic meshwork would perpendicularly pull on junctional complexes, producing a net zero parallel force which will have no effect on junctional length.

The second issue is the $300 \mathrm{~nm}$ problem. Bipolar myosin II minifilaments are $>300 \mathrm{~nm}$ long and geometrically cannot close the junction. If each linear junction has only one myosin II minifilament, a cell with 5 sides will be stuck with a hole $>500 \mathrm{~nm}$ in diameter at 
the end of junction constriction. In Drosophila, apical constriction is arrested with a hole during wound healing, which must be closed by protrusion and cell migration (AbreuBlanco et al., 2012; Wood et al., 2002).

In this study, we have described a 2 actomyosin structures associated with the apical junction: the apical stress fiber and the contractomere (Fig 10A-C). We have described several apical stress fiber organizations, including attachment using head-on or side-on linkers (Fig 10A, lower left). Apical stress fibers are attached to E-cadherin junctions, linking junctions from opposite sides of a cell and across several cells (Fig 10B, upper right \& bottom panels). The second actomyosin structure is a novel actomyosin complex, which we named contractomere (Fig 10A, lower right). The contractomere possesses unique biochemical properties by coupling actin polymerization to myosin II motility. By gliding contractomeres around the cell boundary, epithelial cells within a monolayer can adjust their junctional lengths without having to add new junctional membrane or removing existing junction (Fig 10C, left panel). The contractomere offers a simple solution to junction remodeling by allowing zipping and un-zipping of the junction (Fig 10C, upper right panels). Sliding contractomeres on the plane of the plasma membrane would support junction constriction (Fig 10C, lower right panels); while the junctions surrounding an apical constriction shorten, new junctions are concomitantly formed (Fig 10C, lower right panels). Contractomere motility does not have the $300 \mathrm{~nm}$ geometric constrain and can constrict the junction completely without leaving a hole (Fig 10C, lower right panels). Thus, the contractomere in vertebrates is a novel vertebrate invention evolved using vertebratespecific proteins such as synaptopodin to increase the efficacy of vertebrate epithelial junctions in the maintenance of permeability barrier during wound healing, cell extrusion, and collective cell movements.

A new concept emerged from this study is the origin of force. Force production by the contractomere is an intrinsic process since actin assembly and myosin II ATPase occur directly at the junctional complex (Fig 10D). By contrast, apical stress fibers or cortical actin meshworks are cytoplasmic structures. Force generated by these structures are extrinsic to the junctional complex and must be relayed via linker structures. The existence of 2 independent force-production regimes increases the complexity of mechanoregulation at the apical junction in vertebrate epithelial cells.

\section{Discussion}

We have described a novel molecular machinery at the epithelial apical junction that can couple actin polymerization to myosin II contractility. This junctional complex, which we named contractomere, contains 3 ATPase activities: Arp2/3-dependent actin nucleation, alpha-actinin-4-dependent actin polymerization, and myosin IIB-dependent motility. Contractomere exists as a standalone structure even after the removal of endogenous actin and can be isolated from tissues or cultured epithelial cells. This is in contrast to stress fibers or actomyosin networks where the interactions between actin filaments and myosin II bipolar filaments are necessary for their structural integrity. Contractomere can 
glide along the junction to zip-up the plasma membranes of neighboring cells, creating new cell-cell interface. The movement of contractomeres during cell extrusion allows junction constriction and reorganization of cell boundaries. One of our future goal is to investigate how synaptopodin organizes different junctional proteins to maintain cell-cell adhesion during contractomere movement.

Apical stress fiber, contractomere, and actomyosin meshwork are 3 different forcegenerating structures, contributing to distinct force-dependent processes at the junction. The 3 regimes allow independent regulations and force transmission pathways that are structurally and functionally segregated. Previous studies had shown that myosin IIA structures are primarily controlled by Rho/Rock/MLC whereas myosin IIB structures are mainly regulated by $\mathrm{Ca}^{++} /$calmodulin/MLCK (Chang and Kumar, 2015; Kassianidou et al., 2017; Kuragano et al., 2018; Morin et al., 2014; Totsukawa et al., 2004). Phosphorylation studies using purified myosin II indicate that MLCK phosphorylates myosin IIB more efficiently than ROCK, whereas the reverse is true for myosin IIA (Amano et al., 1996; Sandquist et al., 2006). Furthermore, MLCK is $>50$ times more efficient in phosphorylating MLC than ROCK (Bresnick, 1999). One of our future goals is to determine the contribution of these pathways in the regulation of apical stress fiber and the contractomere.

Myosin II bipolar minifilament has a characteristic dumbbell structure $\sim 300 \mathrm{~nm}$ in length consisted of a bare mid-zone and myosin heads spraying out from 2 ends. In purified junctional membranes, this myosin II filamentous structure had not been observed. Instead, we found a compact macromolecular complex $\sim 150-250 \mathrm{~nm}$ in dimension (Kannan and Tang, 2015). Dissociation of the contractomeric complex using mechanical and biochemically perturbations showed that the coiled-coil tail of myosin II monomer was bound to proteins. These findings are consistent with previous reports by the Svitkina group describing the existence of electron-dense material surrounding the coiled-coil region of unfolded monomeric myosin II (Shutova et al., 2017; Shutova et al., 2014). The coiled-coil region has also been shown to play a role in targeting myosin IIB to secretory granules independent of actin (Milberg et al., 2017). These previous observations and our current results add to the growing number of studies showing that myosin IIB localization to different structures does not require actin binding (Badirou et al., 2014; Beach and Egelhoff, 2009; Fanning et al., 2012; Liu et al., 2016; Rosenberg et al., 2008; Roy et al., 2016). Thus, the interaction between synaptopodin and myosin IIB could potentially recruit myosin IIB to the contractomere. One of our future goals is to determine whether synaptopodin or additional junctional proteins bind to the coiled-coil region of myosin IIB and whether the interaction is responsible for the recruitment of myosin IIB to the contractomere.

The Svitkina group has demonstrated that myosin II monomers are phosphorylated inside cells (Shutova et al., 2017; Shutova et al., 2014). Myosin II phosphorylation activates myosin II, converting myosin II from an inactive autoinhibited state into a constitutively active motor. Consistent with their observations, the Korn group has shown that recombinant myosin II monomers that are in the unfolded state can be phosphorylated in vitro (Liu et al., 2017; Liu et al., 2016; Liu et al., 2018). Collectively, these studies show 
that the motor function of unfolded monomers can be controlled by phosphorylation similar to filamentous myosin II. Importantly, phosphorylated unfolded monomeric myosin II are likely to be constitutively active with actin motility activity.

Multiple phosphorylation sites have been identified on the coiled-coil region of myosin IIB, and phosphorylation of these sites by PKC-zeta and CK-2 promotes disassembly of myosin II minifilaments and formation of myosin II monomers (Dulyaninova and Bresnick, 2013; Even-Faitelson and Ravid, 2006; Juanes-Garcia et al., 2015; Murakami et al., 1998; Murakami et al., 1984; Vicente-Manzanares et al., 2009). PKC-zeta and CK-2 are kinases that regulate the development, stability, and maintenance of epithelial junctions (Dorfel et al., 2013; Eckert et al., 2005; Helfrich et al., 2007; Raleigh et al., 2011). Thus, regulation of myosin IIB by PKC-zeta and CK-2 might contribute to junction maturation by converting myosin II filaments into monomers at the junction. In fact, our results show that apical stress fiber, which is formed by bipolar myosin II filaments, dissolves during junction maturation with concomitant formation of contractomere, formed by monomeric myosin IIB. The dissolution of apical stress fibers is specific at the apical junction because basal stress fibers remain intact, indicating that the apical junction contains regulators for the conversion process. Local regulation at the apical junction would provide an immediate pool of monomeric myosin IIB for the assembly of contractomeres. This is in contrast to the current paradigm that monomeric myosin II is the inactive reserved pool for myosin II minifilament assembly. One of our future goals is to determine the mechanism responsible for converting bipolar filaments into monomeric myosin IIB at the apical junction.

Although the structure, composition, and molecular mechanism of junction contractomere have not been completely elucidated in this study, we have provided experimental evidence and logistic arguments for its existence as a novel membrane organelle. We demonstrated that the contractomere uses a novel mechanism that harnesses energy from actin polymerization and myosin ATP cycle to support contractile functions at the apical junction. The ability of the contractomere to move on the plane of the plasma membrane allows new cell-cell adhesion interface to form between neighboring cells. We propose that the contractomere is an essential contractile machinery with pivotal roles in the maintenance of epithelial integrity during wound healing, cellular turnover, and intercellular arrangement. The discovery of the contractomere reveals a missing piece in the molecular mechanism of apical junction and contributes to understanding of epithelial behaviors in vertebrates. 


\section{Materials and Methods}

\section{Antibodies and reagents}

Primary antibodies were purchased from commercial sources and custom-generated. Rabbit and goat polyclonal antibodies to synaptopodin were raised against synthetic peptides corresponding to 4 regions of human synaptopodin X1 (NCBI Reference Sequence: XP_016864497.1): aa164-180 (PSSNSRGVQLFNRRRQR), aa675-684 (QQESAPRDRA), aa899-918 (SPRAKQAPRPSFSTRNAGIE), and aa1123-1143 (CPRGWNGSLRLKRGSLPAEAS). The peptides were synthesized and coupled to KLH before injection into the animals (Pacific immunology). Serum reactivity to the peptides were assessed by ELISA (Pacific immunology). Rabbit polyclonal antibodies to a-actinin-4 were raised in house against a synthetic peptide corresponding to aa 8-24 of human aactinin-4, NQSYQYGPSSAGNGAGC, that has been coupled to KLH. Antibodies to aactinin-4 (sc-3933495, mouse), beta-catenin (sc-7963, mouse), synaptopodin (sc-515842, mouse; sc-21537, goat), a-catenin (sc-9988, mouse), p120 (sc-13957, rabbit), vinculin (sc5573, rabbit), myosin IIA (sc-47201, rabbit), myosin IIB (sc-376942, mouse) were purchased from Santa Cruz Biotechnology. Rabbit polyclonal antibodies to myosin IIA (19098) and myosin IIB (19099) were purchased from Biolegend. Antibodies to phosphomyosin light chain 2 (Thr18/Ser19) were purchased from Cell Signaling Technology (\#3674, rabbit). Secondary antibodies were purchased from Bio-Rad Laboratories (HRP goat anti-rabbit), Santa Cruz Biotechnology (HRP rabbit anti-goat and goat- anti-mouse), Life Technologies/Invitrogen Alexa 488 donkey anti-mouse, Alexa 568 donkey anti-rabbit, Alexa 647 donkey anti-goat).

Protease inhibitors used for cell extraction and membrane preparation, leupeptin, Pefabloc, E-64, antipain, aprotinin, bestatin, and calpain inhibitors I and II, were purchased from A.G. Scientific, Inc. Rhodamine, rhodamine green, Latrunculin B, cytochalasin D, FITC-phalloidin, TRITC-phalloidin, blebbistatin, were purchased from Sigma. Alexa 647phalloidin and Alexa 350-phalloidin were purchased from ThermoFisher/LifeTechnologies. Stock solutions of latrunculin B (5 mM), cytochalasin D (2 mM), blebbistatin (5 mM), phalloidin were prepared in dimethylsulfoxide.

\section{DNA constructs}

Synaptopodin A was synthesized by Genscript using the coding sequence of human synaptopodin (NCBI Reference Sequence: NP_009217.3) and was subcloned by Genscript into HINDIII and Xho1 sites of the blasticidin-selectable mammalian expression vector pcDNA6 myc-HisA (Invitrogen) containing an N-terminal sstFRET (Meng and Sachs, 2011) and C-terminal myc and His tags.

sstFRET consists of a spectrin repeat flanked by cerulean and venus fluorescent proteins (tccgtgagcaagggcgaggagctgttcaccggggtggtgcccatcctggtcgagctggacggcgacgtaaacggccaca agttcagcgtgtccggcgagggcgagggcgatgccacctacggcaagctgaccctgaagctgatctgcaccaccggcaag ctgcccgtgccctggcccaccctcgtgaccaccctgggctacggcctgcagtgcttcgcccgctaccccgaccacatgaagc agcacgacttcttcaagtccgccatgcccgaaggctacgtccaggagcgcaccatcttcttcaaggacgacggcaactacaa 
gacccgcgccgaggtgaagttcgagggcgacacctggtgaaccgcatcgagctgaagggcatcgacttcaaggaggac ggcaacatcctggggcacaagctggagtacaactacaacagccacaacgtctatatcaccgccgacaagcagaagaacg gcatcaaggccaacttcaagatccgccacaacatcgaggacggcggcgtgcagctcgccgaccactaccagcagaacac ccccatcggcgacggcccotgctgctgcccgacaaccactacctgagctaccagtccaaactgagcaaagaccccaacg agaagcgcgatcacatggtcctgctggagttcgtgaccgccgccgggatcactctcggcatggacgagctgtacaagtccgg actcagatctggaggcttccacagagatgctgatgaaaccaaagaatggattgaagagaagaatcaagctctaaacacag acaattatggacatgatctcgccagtgtccaggccctgcaacgcaagcatgagggcttcgagagggaccttgcggctctcggt gacaaggtaaactcccttggtgaaacagcagagcgcctgatccagtcccatcccgagtcagcagaagacctgcaggaaaa gtgcacagagttaaaccaggcctggagcagcctggggaaacgtgcagatcagcgcaaggcaaagggaggcgtgaattcc atggtgagcaagggcgaggagctgttcaccggggtggtgcccatcctggtcgagctggacggcgacgtaaacggccacaa gttcagcgtgtccggcgagggcgagggcgatgccacctacggcaagctgaccctgaagttcatctgcaccaccggcaagct gcccgtgccctggcccacctcgtgaccaccctgacctggggcgtgcagtgcttcgcccgctaccccgaccacatgaagca gcacgacttcttcaagtccgccatgcccgaaggctacgtccaggagcgcaccatcttcttcaaggacgacggcaactacaag acccgcgccgaggtgaagttcgagggcgacaccctggtgaaccgcatcgagctgaagggcatcgacttcaaggaggacg gcaacatcctggggcacaagctggagtacaacgccatcagcgacaacgtctatatcaccgccgacaagcagaagaacgg catcaaggccaacttcaagatccgccacaacatcgaggacggcagcgtgcagctcgccgaccactaccagcagaacacc cccatcggcgacggcccogtgctgctgcccgacaaccactacctgagcacccagtccaagctgagcaaagacccaacg agaagcgcgatcacatggtcctgctggagttcgtgaccgccgccgggatcactctcggcatggacgagctgtacaag).

ShRNA for canine synaptopodin cloned into puromycin-selectable pRS mammalian expression vector (Origene) has previously been described (Kannan and Tang, 2015).

Plasmids for alpha-actinin-4 FRET-based tension sensors were synthesized and sequenced by Genscript. Alpha-actinin-4 sstFRET408 was constructed by inserting the sstFRET module between 408aa and 409aa of human alpha-actinin-4 (XP_016882820.1), between the first and second spectrin repeats, and cloned into the blasticidin selectable pUNO expression vector (Invivogen). Alpha-actinin-4 sstFRET522 was constructed by inserting the sstFRET module between 408aa and 409aa of human alpha-actinin-4 (XP_016882820.1), between the second and third spectrin repeats, and cloned into pUNO. Plasmids for alpha-actinin-1 FRET-based tension sensors were kindly provided by Fanjie Meng \& Frederick Sachs, Physiology and Biophysics Department, SUNY at Baffalo (Meng and Sachs, 2011). Alpha-actinin-1-M-sstFRET containing the sstFRET module inserted between 300aa and 301aa within the first spectrin repeat of human alpha-actinin-1 (P12814.2) was in the neomyocin selectable pcDNA3.1 expression vector (Invitrogen/ThermoFisher). Actinin-C-sstFRET containing the sstFRET module added to the C-terminus of human alpha-actinin-1 (P12814.2) was in pcDNA3.1.

Plasmids for bacterial expression of recombinant alpha-actinin-4 was generated from PCR products using the coding sequence of human alpha-actinin-4 (NP_004915.2) kindly provided by Martin Pollak. Alpha-actinin-4 full length (1-911aa), actin binding domain (1270aa), and spectrin repeats/EF hands (271-911aa) were subcloned into EcoR1 and Xho1 sites in the kanamycin-selectable bacterial expression vector pET30a+ containing an Nterminal 6His tag (EMD4Biosciences) and the ampicillin-selectable bacterial expression vector PMAL4cx with $\mathrm{N}$-terminal maltose-binding protein. 


\section{Cell culture \& transfection}

Madin-Darby canine kidney (MDCK) cells were originally from Kai Simons lab (EMBL, Germany) and a gift from Barry Gumbiner (University of Washington). The cells have been authenticated by staining of E-cadherin and ZO-1 using antibodies that only recognize the canine proteins, $\mathrm{RR} 1$ for E-cadherin and $\mathrm{R} 40.76$ for ZO-1. The cells are free from mycoplasma contamination as determined by the original source. MDCK cells were maintained in MEM/Earle's balanced salt solution supplemented with $25 \mathrm{mM}$ Hepes and $5 \%$ fetal bovine serun (FBS) in a 37 degree incubator in the presence of $5 \% \mathrm{CO}$. Media was changed every 2-3 days. Cell stocks for parental and transfected cells are stored in freezing media (normal growth media, 20\% FBS, 10\% DMSO) in liquid nitrogen. MDCK cells were grown on tissue culture plastic dishes. For live-cell imaging, wound healing, and immunofluorescence, cells were plated on No.2 VistaVision cover glasses (VWR). For hydraulic chamber experiments and immunofluorescence, cells were plated on $12 \mathrm{~mm}$ polyester Transwell-clear with 0.4 um pores (Corning). Confluent monolayer of MDCK epithelial cells polarized on semi-permeable Transwell support for $>2$ weeks reaches a steady-state that generate junctions with strong cell-cell adhesion.

For transfection, cells were incubated in a 1:1 mixture of DNA/Polyjet DNA transfection reagent for 5-18 hours according to the manufacturer's protocol (SignaGen Laboratories). Expression of plasmid DNA was selected using G418, puromycin, or blasticidin for 10-14 days. Stable expressing clonal cell lines were obtained as published (Kannan and Tang, 2015). Briefly, antibiotic resistant clonal cell lines were expanded and assessed for knockdown efficiency and protein expression by western blot and immunofluorescence. Clonal cell lines with homogeneous knockdown phenotype were used for a second round of transfection with ShRNA. Secondary clonal cell lines were expanded and assessed for knockdown efficiency by western blot and immunofluorescence. Clonal cell lines with knockdown efficiency of $>80 \%$ were used for this study.

For live-cell imaging of alpha-actinin-4 sstFRET522, alpha-actinin-1-M-sstFRET and alphaactinin-1-C-sstFRET, and synaptopodin-venus cell lines were expanded and assessed for expression using live-cell microscopy. For mixed population of cells expressing alphaactinin-4 sstFRET408, antibiotic resistant cells were pooled and used for live-cell imaging 14-21 days post-transfection or frozen until use.

\section{Wound closure assay}

MDCK confluent monolayers form domes when grow on non-permeable glass coverslips $>3-4$ weeks. After dome formation, monolayers were rinsed 3 times with calcium-free phosphate-buffered saline and incubated with calcium-free serum-free MEM media at 37 degrees for 10-15 min until the cells forming the domes were detached from the monolayers. Immediately after that, the calcium-free media was removed and fresh prewarmed normal growth media with or without cytochalasin $\mathrm{D}$ (at a final concentration of 0.2 $\mathrm{uM}$ ) or blebbistatin (at a final concentration of $60 \mathrm{uM}$ ) was gently added to the dishes containing the coverslips with attached MDCK monolayers that now have holes. Cell 
monolayers were incubated at 37-degree incubator for wound closure to proceed. At one hour after initiation of wound healing, cell monolayers on coverslips were processed immediately for immunofluorescence.

\section{Staining and immunofluorescence of cell}

Cells grown on Transwell-Clear (Corning) were used in localization studies. For immunofluorescence, cells were rinsed twice in $150 \mathrm{mM} \mathrm{NaCl} / 2 \mathrm{mM} \mathrm{CaCl} / 2 \mathrm{mM} \mathrm{MgCl} / 20$ $\mathrm{mM}$ Hepes, $\mathrm{pH} 7.8$ and fixed in $1 \%$ formaldehyde $/ 150 \mathrm{mM} \mathrm{NaCl} / 2 \mathrm{mM} \mathrm{CaCl} / 2 \mathrm{mM}$ $\mathrm{MgCl}_{2} / 20 \mathrm{mM}$ Hepes, $\mathrm{pH} 7.8$ at $4^{\circ} \mathrm{C}$ for 1 hour. The reaction was quenched with Tris in quenching buffer $(0.05 \%$ Triton $\mathrm{X}-100 / 50 \mathrm{mM}$ Tris/100 mM NaCl/20 mM Hepes, $\mathrm{pH} 8.0)$ for 3 hours. The Transwell with fixed cells were rinsed in immunofluorescence staining buffer (0.05\% Triton X-100/100 mM NaCl/20 mM Hepes, $\mathrm{pH} 7.5)$, the cells were incubated with primary antibodies $(1 \mathrm{ug} / \mathrm{ml})$ in staining buffer overnight. After rinsing in staining buffer three times, the cells were incubated in secondary antibodies in staining buffer for 90 min. Then, the cells were rinsed three times in staining buffer and incubated with fluorescently labeled phalloidin or Hoechst 33528 for 60 min. Finally, the cells were rinsed three times in staining buffer and post-stain fixed with 1\% formaldehyde in staining buffer for 3 hours. Transwell filters were excised using a razor blade and mounted with No.1 glass coverslips on glass slides with ProLong Glass antifade (Invitrogen). Stained cells with antifade were allowed to cure for 48 hours before used for imaging.

\section{Image acquisition of fixed cell}

Images were collected in 200-nm steps using Axio Imager.Z2m microscope equipped with Apotome.2 (Carl Zeiss) and X-cite 120 LED (Lumen Dynamics). For Optical Sectioning Structured Illumination Microscopy (OS-SIM), 7 phases/images were collected per each constructed image using either alpha Plan-Apochromat 100x/NA1.46 Oil DIC M27 or PlanApochromat 40X/NA1.3 Oil DIC M27 objectives (Carl Zeiss) and a 4K ORCA-Flash4.0 V2 digital CMOS camera with 6.5 um x 6.5 um pixel size (Hamamatsu Photonics). Widefield optical $z$ images were deconvolved using the Zen2 pro deconvolution module. Low magnification wide field images were collected using Plan Apochromat 20X/0.8 objectives (Zeiss). Images for figures $1 \mathrm{~A}, 1 \mathrm{~B}$, and 7A were collected using a 2K Optimos CMOS camera with 6.5 um x 6.5 um pixel size (Qimaging, Photometrics). Composite images were generated using Image $(\mathrm{NIH})$ and Zen (Carl Zeiss) softwares. For figure generation, images were cropped, contrasted, and scaled using Photoshop software (Adobe) before importing into Illustrator (Adobe).

\section{Image analysis}

All images were corrected for chromatic shift on the X, Y, Z-axes for each fluorescence channel before were used for analysis. Quantitation of actin (phalloidin) and junction proteins (immunofluorescence intensity) was performed in Image $(\mathrm{NIH})$ or Zen (Carl Zeiss) using unprocessed original single optical z-slice images. A defined area was used to compare the signal intensity of actin (phalloidin) and immunofluorescence of junctional 
proteins. All measured intensities were subtracted from background signal (an area with no cells or within the cytoplasm) before used for statistical analyses and calculation of intensity ratios. All experiments had been repeated at least three times. At least 6 data set from each experiment were collected.

All analyses were performed using KaleidaGraph software (Synergy). Each junctional region is outlined manually with a freehand drawing tool. The mean pixel intensity of each defined junctional region is used for comparison of junction localization of individual junctional protein. For calculation of Pearson's correlation coefficient $R$, intensities of individual pixel within the defined junctional region were used and each pixel corresponds to $45 \times 45 \mathrm{~nm}$ of the imaged sample. All p-values were calculated using non-paired student t-tests. Linear regression was fitted using original data points. Line intensity graphs were generated in Excel (Microsoft) using pixel intensities from original images. For measurement of junctional length, distance between cell vertices, cell perimeter, wound perimeter and area, a free-drawing tool and a line tool were used to trace outlines and draw straight lines.

\section{Live-cell imaging setup}

For live-cell imaging, glass coverslips were soaked in $100 \%$ ethanol, and sterilized under UV for $60 \mathrm{~min}$. Sterilized coverslips were coated with $20 \mathrm{ug} / \mathrm{ml}$ collagen IV in phosphate buffered saline for $60 \mathrm{~min}$ and used immediately for plating of cells. Cells grown on collagen-coated glass coverslips were mounted up-side-down onto an in-house fabricated polycarbonate chamber with a deep well for media for long-term imaging. Imaging was performed in FluoroBrite/DMEM (Gibco) media containing 10\% fetal bovine serum and 10 $\mathrm{mM}$ HEPES, $\mathrm{pH}$ 7.5. Sample temperature was maintained at 35 degrees Celsius on a heated stage and an objective heater (PeCon). Images were collected using ORCAFlash4.0 (Hamamatsu Photonics) or Optimos (Qimaging) mounted onto Axio Imager.Z2m (zeiss) with X-cite 120 LED (Lumen Dynamics).

\section{Live-cell imaging of alpha-actinin FRET and synaptopodin}

Alpha-actinin tension sensors were imaged using the Gemini dual-view system (Hamamatsu Photonics) equipped with excitation filter for Cerulean fluorescence protein (CeFP) and emission filters for Cerulean fluorescence protein (CeFP) and Venus fluorescence protein (VFP). Simultaneous acquisition of images for CeFP and VFP) emission were obtained using a Plan-Apochromat 40x/NA1.3 Oil DIC M27 objective (Zeiss). Junctional intensities of CeFP and VFP were used to calculate the FRET index $($ EmVFP)/(EmCeFP + EmVFP), which is shown as FRET/(CeFP+FRET). Briefly, the CeFP and VFP channels were overlay on top of each other using a macro written within the Zen2 software (Carl Zeiss). Each junctional region is outlined manually with a freehand drawing tool in the Zen2 imaging tool (Zeiss). The intensities of the junctional signal were measured and subtracted from background signal (an area with no cells or within the cytoplasm) before used for the calculation of the FRET index. For movie generation, individual images of cropped cells were imported into QuickTime (Apple) to 
generate movie files. Composite images were generated using ImageJ $(\mathrm{NIH})$ or Photoshop (Adobe). Individual cell diameter and junction for each image were measured using image $\mathrm{J}(\mathrm{NIH})$ or Zen (Carl Zeiss).

\section{Western blot}

For comparison of young and mature monolayers, confluent monolayers of cells were trypsinized and replated at high confluent density $\left(10^{7}\right.$ cells per $\left.10 \mathrm{~cm}\right)$. Cells were allowed to form cell-cell interactions for 2 days (young) or 7 days (mature). Total cell lysates were obtained by solubilizing the cells in SDS-PAGE sample buffer containing 25 $\mathrm{mM}$ dithiothreitol, 2\% SDS, $50 \mathrm{mM}$ Tris-Cl, 5\% glycerol, $\mathrm{pH} 8.8$ and protease inhibitors (10 ug/ml Leupeptin, 1 $\mathrm{mg} / \mathrm{ml}$ Pefabloc, $10 \mathrm{ug} / \mathrm{ml}$ E-64, 2 ug/ml antipain, $2 \mathrm{ug} / \mathrm{ml}$ aprotinin,50 $\mathrm{ug} / \mathrm{ml}$ bestatin, $20 \mathrm{ug} / \mathrm{ml}$ calpain inhibitors I \& $10 \mathrm{ug} / \mathrm{ml}$ calpain inhibitor II). For western blot of phosphorylated myosin light chain, cell extraction was carried out as above with the addition of a phosphatase inhibitor cocktail (Simple Stop 1 \#GB-450) purchased from Gold Biotechnology, USA. Biorad DC detergent compatible protein assay was used to determine total protein concentration in cell lysates. Equal protein amounts of cell lysates were used for comparison of junctional protein. SDS-PAGE was performed using 8-16\% gradient gel and transferred to nitrocellulose paper using the Transblot (BioRad). Western blots were carried out using iBind with $1 \mathrm{ug} / \mathrm{ml}$ primary antibodies and $1 \mathrm{ug} / \mathrm{ml}$ secondary antibodies following iBind protocol (Invitrogen/ThermoFisher).

\section{Recombinant protein expression, purification, and fluorophore labeling}

For expression of recombinant proteins, cDNA plasmids were transformed into Rosetta DE3 E. Coli cells containing tRNA's for "universal" translation" (Novagen) maintained under chloramphenicol. For recombinant 6 -His tagged $\alpha$-actinin-4, actin binding domain, and spectrin repeats/EF hands, protein expressions were induced with $500 \mathrm{uM}$ Isopropyl $\beta$-D-1-thiogalactopyranoside for 8 hours at $25^{\circ} \mathrm{C}$ in LB containing chloramphenicol. Cells were centrifuged at $6000 \mathrm{xg}$ for $15 \mathrm{~min}$ and resuspended in $20 \mathrm{mM} \mathrm{NaCl}, 20 \mathrm{mM} \mathrm{HEPES}$, $\mathrm{pH} 7.8$ in the presence of $5 \mathrm{mg} / \mathrm{ml}$ lysozyme. After one freeze-thaw cycle, lysed cells were centrifuged at $100,000 \mathrm{xg}$ for $30 \mathrm{~min}$. Supernatant was loaded onto a nickel column (Qiagen). The column was washed with 20 bed volumns of $500 \mathrm{mM} \mathrm{NaCl}, 25 \mathrm{mM}$ Imidazole, and $20 \mathrm{mM}$ HEPES, $\mathrm{pH}$ 7.8. The recombinant proteins were eluted with 10 bed volumes of $500 \mathrm{mM} \mathrm{NaCl}, 500 \mathrm{mM}$ Immidazole, $20 \mathrm{mM}$ HEPES, pH7.8. Eluted proteins were concentrated using centricon and purified by gel filtration using superdex 200 in 150 $\mathrm{NaCl}, 20 \mathrm{mM}$ HEPES, $10 \mathrm{mM} \beta$-mercaptoethanol. Proteins were either frozen or used immediately for labeling. Recombinant alpha-actinin-4 proteins were labeled on cysteine using maleimide-activated oregon green at a ratio of 5 fluorophores for every $\alpha$-actinin at room temperature for one hour. Labelled proteins were separated from free dyes by gel filtration using superdex 200 in $150 \mathrm{NaCl}, 20$ mM HEPES, 10 mM $\beta$-mercaptoethanol.

For expression of heterodimeric alpha-actinin-4 consists of one monomer of full-length and one monomer of spectrin-repeats/EF hands, plasmid pet30a+ with full-length alpha-actinin4 and plasmid pMAL-4cx with spectrin repeats/EF hands were transformed together into 
Rosetta DE3 cells. Cells expressing both constructs were selected with ampicillin and kanamycin and maintained in LB containing $10 \mathrm{mM}$ glucose to suppress the expression of MBP-fusion protein. To induce protein expression, 200 uM Isopropyl $\beta$-D-1-

thiogalactopyranoside was added to the LB growth media for 8 hours at 25 degrees. Cells were centrifuged at $6000 \mathrm{xg}$ for $15 \mathrm{~min}$ and resuspended in $20 \mathrm{mM} \mathrm{NaCl}, 20 \mathrm{mM} \mathrm{HEPES}$, $\mathrm{pH} 7.8$ in the presence of $5 \mathrm{mg} / \mathrm{ml}$ lysozyme. After one freeze-thaw cycle, lysed cells were centrifuged at 100,000 xg for $30 \mathrm{~min}$. Supernatant was loaded onto an amylose column 3 times (NED), washed with 20 bed volumes of $100 \mathrm{mM} \mathrm{NaCl}, 20 \mathrm{mM}$ HEPES, pH7.8, and eluted with $10 \mathrm{mM}$ maltose in $100 \mathrm{mM} \mathrm{NaCl}, 20 \mathrm{mM}$ HEPES, pH7.8. The eluted fraction was loaded onto a nickel column (Qiagen). The column was washed with 20 bed volumns of $500 \mathrm{mM} \mathrm{NaCl}, 25 \mathrm{mM}$ Imidazole, and $20 \mathrm{mM}$ HEPES, pH 7.8. The recombinant proteins were eluted with 10 bed volumes of $500 \mathrm{mM} \mathrm{NaCl}, 500 \mathrm{mM}$ Immidazole, $20 \mathrm{mM}$ HEPES, pH7.8. Eluted proteins were concentrated using centricon and purified by gel filtration using superdex 200 in $150 \mathrm{NaCl}, 20 \mathrm{mM}$ HEPES, $10 \mathrm{mM} \beta$-mercaptoethanol.

Actin was purified from rabbit skeletal muscle as described (Brieher et al., 2004). Actin was labelled on lysine residues using two NHS activated rhodamine for every actin molecule for one hour at room temperature as described (Brieher et al., 2006). After 'labeling, actin filaments were pelleted at 100,000 X g for 30 minutes, resuspended in $\mathrm{G}$ buffer and dialyzed exhaustively against G buffer (0.2 mM ATP, $0.2 \mathrm{mM} \mathrm{CaCl}_{2}, 5 \mathrm{mM}$ Trs$\mathrm{HCl}, \mathrm{pH} 8,1 \mathrm{mM} \beta$-mercaptoethanol). This procedure typically labeled actin to $50-80 \%$. Aliquots of proteins were snap-freeze in liquid nitrogen and stored at -80 degrees until use. Protein concentrations were determined by Bradford assay (BioRad).

\section{Purification of junction-enriched membrane}

Junction-enriched membranes were prepared as described (Tang and Brieher, 2012). Briefly, frozen rat livers (Pelfrez) were thawed in 2 volumes of $10 \mathrm{mM}$ Hepes, pH8.5/10 mM DTT. Protease inhibitors (see above) were added and the livers were briefly blended in a Waring blender $(5 \times 15 \mathrm{sec})$. The liver slush was filtered through 4 layers of cheesecloth to obtain the total liver homogenate. Total liver homogenate was centrifuged at $1000 \mathrm{xg}$ for $30 \mathrm{~min}$. The pellet was homogenized in $10 \mathrm{mM}$ Hepes, pH8.5/10 mM DTT in a dounce homogenizer and centrifuged at $100 \mathrm{xg}$ for $30 \mathrm{~min}$. The supernatant was collected and centrifuged at $1000 x \mathrm{x}$ for $30 \mathrm{~min}$. The membrane pellet contains the majority of actin assembly activity and is frozen at -80 degrees before further purification immediately before use. The day of the experiment, membranes were thawed on ice, diluted 1:1 with $10 \mathrm{mM}$ HEPES, pH 8.5 supplemented with $10 \mathrm{mM}$ DTT and homogenized through a $25 \mathrm{G}$ needle. The homogenates were spun through a $20 \%$ sucrose pad for 10 minutes at 16,000 $\mathrm{xg}$. The supernatant was discarded, and the pellet were resuspended with $10 \mathrm{mM}$ HEPES, pH 8.5 supplemented with $10 \mathrm{mM}$ DTT. The homogenate was spun through a $20 \%$ sucrose pad for $15 \mathrm{~min}$ at $1000 \mathrm{xg}$. The pellet was discarded, and the supernatant was spun through a $20 \%$ sucrose pad for $15 \mathrm{~min}$ at $16,000 \mathrm{xg}$. The membrane pellet contains junction-enriched plasma membrane fragments as determined by western blots and immunostaining (Tang and Brieher, 2012). 


\section{Membrane actin assembly and immunofluorescence}

Actin assembly reactions were performed in actin polymerization buffer $(50 \mathrm{mM} \mathrm{KCl}, 2 \mathrm{mM}$ EGTA, $2 \mathrm{mM} \mathrm{MgCl}_{2}$, and $100 \mathrm{mM}$ Hepes, $\mathrm{pH}$ 7.8) supplemented with $2 \mathrm{mM}$ buffered ATP, $\mathrm{pH}$ 8. A standard $20-\mu \mathrm{L}$ reaction consists of $\sim 15 \mu \mathrm{g}$ of total proteins from the purified junctional membrane fraction and $1 \mu \mathrm{M}$ rhodamine or Oregon green-labeled monomeric actin. Actin polymerization was allowed to carry out at room temperature for 30-60 min.

For reconstitution assays, purified membranes were stripped with high salt $(500 \mathrm{mM} \mathrm{NaCl}$, $2 \mathrm{mM} \mathrm{MgCl}$, 2 mM EGTA, 20 mM Hepes, pH 7.8, and 10 mM DTT), TX-100 or CHAPS (in $50 \mathrm{mM} \mathrm{NaCl}, 2 \mathrm{mM} \mathrm{MgCl}$, 2 mM EGTA, $20 \mathrm{mM}$ Hepes, $\mathrm{pH}$ 7.8, and $10 \mathrm{mM}$ DTT) on ice for 1 hour. Stripped membranes were collected by centrifugation through a $20 \%$ sucrose cushion at $10,000 \mathrm{~g}$ for $10 \mathrm{~min}$. Purified proteins (labelled and unlabeled a-actinin-4) were allowed to bind to stripped membranes for $1 \mathrm{hr}$ at room temperature. Unbound proteins were removed by spinning membranes through a 20\%w/w sucrose cushion at 10,000 $\mathrm{g}$ for $10 \mathrm{~min}$. The final reconstitution reaction consists of $\sim 8 \mu \mathrm{g}$ of total protein from stripped membranes, $0.5 \mu \mathrm{M}$ fluorescently labeled monomeric actin, and purified proteins (aactinin-4) and was carried out at room temperature for $30 \mathrm{~min}$.

For immunofluorescence of membranes, actin assembly assay was performed, and membranes were collected by centrifugation through a $20 \%$ sucrose cushion at $10,000 \mathrm{~g}$ for 10 min. The purified membranes with incorporated actin were incubation with primary antibodies in the presence of $0.1 \% \mathrm{TX}-100$ in actin assay buffer for 2 hours. The membranes were spun through a $20 \%$ sucrose cushion and resuspended in $0.1 \%$ TX-100 in assay buffer. The membranes were incubated with secondary antibodies for 2 hours, spun through a $20 \%$ sucrose cushion, and resuspended in 0.1\% TX-100 in assay buffer.

The membranes were mounted on a glass slide and covered with a No.1.5 glass coverslip before imaging. Images were obtained using a Plan-Apochromat 63x/NA1.4 Oil DIC M27 objective (Carl Zeiss) attached to an Axio Imager (Carl Zeiss) equipped with ORCAER CCD camera with 6.45 um X 6.45 um pixel size (Hamamatsu Photonics) and the Colibri Illumination System (Carl Zeiss).

\section{Electron Microscopy}

For visualization of actin with the junctional complex on membrane sheet, actin assembly reaction was performed using rhodamine-labeled monomeric actin at a final concentration of $1 \mu \mathrm{M}$. Actin polymerization was allowed to carry out at room temperature for $60 \mathrm{~min}$. The reaction was purified by spinning through a $20 \%$ sucrose cushion. The membrane with incorporated actin was resuspended in $10 \mathrm{uL}$ of actin polymerization buffer. $4 \mathrm{uL}$ of the resuspended membranes was used for wide-field light microscopy as described above. $5 \mathrm{uL}$ of the resuspended membranes was processed for electron microscopy analysis. The resuspended membranes were allowed to attach onto glow-discharged carbon-coated grids for $10 \mathrm{~min}$. Unbound membranes were removed by washing the EM grids three times with assembly buffer. The membranes were negatively stained with $2 \%$ uranyl 
acetate and excess stain was remove immediately. The grids were allowed to air dry for $10 \mathrm{~min}$ and stored until image under an electron microscope. Images were collected with a JEOL 2100EX at $120 \mathrm{kV}$ using a $2 \mathrm{~K} \times 2 \mathrm{~K}$ CCD camera (UltraScan; Gatan, Inc.). For figure generation, images were cropped, contrasted, and scaled using Photoshop software (Adobe) before importing into Illustrator (Adobe).

\section{Force application in cell monolayer}

All hydraulic pressure experiments were performed in a 37-degree Celsius room. Cells grown on Transwell-Clear (Corning) were used in hydraulic pressure experiments. Hydraulic chambers and adaptors were fabricated from medical-grade stainless steel by mechanical engineering machine shop at University of Illinois, Urbana-Champaign as described (cite). Transwell filter cups were mounted onto pressure chamber, held in place to a lid by a screw and a top. Hydraulic pressure was applied to the basal compartment via a syringe hooked up to a syringe pump into the basal chamber that is filled with cell culture media and sealed with an O-ring between the pressure chamber and the Transwell cup. Pressure was monitor through an outlet from the basal pressure chamber to a pressure gauge. An apical adaptor is mounted onto the apical chamber of the Transwell cup that is filled with cell culture media and sealed with an O-ring. An outlet from the apical adaptor is either exposed to ambient pressure or connected via a bifurcation to a pressure gauge and a syringe hooked up to a syringe pump. Cyclic, pulsatile, or sustained pressures were applied to cell monolayers by programmable infuse/withdrawal syringe pumps (Lagato SPLG270). Cyclic pressure is applied at 30 cycles per min or otherwise specified. Pulsatile pressure is applied at 6 cycles per min or otherwise specified. After experimental procedure, the Transwells were immediately rinsed 3 times in cold PBS on ice and processed for immunofluorescence.

\section{Data Availability}

All data are available from the corresponding author.

\section{Supplementary materials}

(1) 9 Supplementary Figures.

(2) 15 videos. 


\section{Acknowledgements}

We thank Nivetha Kannan, Cameron Shahnazi, Kevin Huang, Kyle Sherman, Bobby Knier, Rafael Anorga for help with protein gels, molecular biology, generation and maintenance of cell lines, and molecular biology. Electron microscopy was carried out in part in the Frederick Seitz Materials Research Laboratory Central Facilities, University of Illinois. The pressure chamber was built by the mechanical engineering machine shop at the University of Illinois, Urbana-Champaign. This work is funded by the National Institute of Health (R01-DK098398 to Vivian W Tang and R01-GM106106 to William M Brieher). The authors declare no competing financial interests.

\section{Author Contribution}

Vivian M Tang performed experiments, analyzed data, wrote manuscript and provided funding. William M Brieher provided all actin reagents and funding. Timothy Morris generated synaptopodin reagents for cell experiments. Eva Sue generated alpha-actinin-4 cell lines for FRET experiments. Caleb Geniesse purified junctional membranes and generated alpha-actinin-4 proteins for in vitro experiments. 


\section{Legend}

Figure 1. Superresolution immunofluorescence microscopy of 2 actomyosin structures at the apical junction of MDCK epithelial cell monolayers. (A) Apical stress fibers are characterized by alternating pattern of synaptopodin and myosin IIB. (B) Apical stress fibers are inserted at cell-cell adhesions marked by synaptopodin and alpha-actinin4. (C) Apical stress fibers are labelled as Type I actomyosin structure with alternating synaptopodin and myosin IIB organization. Type I apical stress fiber disintegrates upon junction maturation which coincides with the appearance of Type II actomyosin structure containing synaptopodin and myosin IIB . Basal stress fibers have alternating pattern of synaptopodin and myosin IIB organization similar to Type I apical stress fibers.

Figure 2. Superresolution immunofluorescence microscopy showing the assembly and disassembly of $\mathbf{2}$ actomyosin structures during junction maturation. (A) Apical stress fiber, corresponding to type I actomyosin organization shown in the left panel and the graph, has alternating myosin IIB and synaptopodin pattern and lies parallel to Ecadherin junction. Orange arrowheads point to the end of an apical stress fiber marked by E-cadherin. White arrowheads point to the junctional region where synaptopodin is in close proximity to E-cadherin. White long arrow shows the junctional length used for $\mathrm{x}$ axis of the graph. (B) Apical stress fiber is inserted head-on at E-cadherin junction, marked by orange arrowhead. Synaptopodin overlaps with E-cadherin at the insertion point of apical stress fiber, in white circle. White arrowheads mark sites where synaptopodin is in close proximity to or overlapping with E-cadherin. (C) Disassembly of apical stress fibers in maturing junction and loss of alternating pattern of synaptopodin and myosin IIB as shown in graph. The disassembly of apical stress fiber coincides with the formation of type II actomyosin structures containing myosin IIB, synaptopodin, and alphaactinin-4, as shown in graph. White circles in the left panels show type II structures. White arrowheads mark the colocalization of myosin IIB, synaptopodin, alpha-actinin-4. White long arrow shows the junctional length used for x-axis of the graph. (D) Apical stress fibers disappear upon junction maturation whereas type II actomyosin structures are prominent at mature apical junctions. Left panel are $\mathrm{X}-\mathrm{Y}, \mathrm{Y}-\mathrm{Z}$, and $\mathrm{X}-\mathrm{Z}$ views of mature polarized MDCK monolayers. Small white boxed areas are enlarged to show colocalization of myosin IIB, synaptopodin, and alpha-actinin-4. (E) Actin accumulates at type II actomyosin structure, circled in white, colocalizing with myosin IIB, synaptopodin, and alpha-actinin-4 marked by white arrowheads. Graph shows the absence of apical stress fiber and the presence type II structure. White long arrow shows the junctional length used for $\mathrm{x}$-axis of the graph.

Figure 3. Live-imaging of synaptopodin showing 2 apical actomyosin structures and basal stress fibers in MDCK cell monolayers. (A) Apical stress fibers are outlined by aligned and repeated synaptopodin densities, circled in blue, yellow, and green. Apical stress fibers are inserted at the junction via synaptopodin linkers, highlighted in pink, using 2 configurations, either head-on (type la) or side-on (type lb). Head-on anchors insert ends of stress fibers at the junctions. Apical stress fibers can link junctions from opposite side of the cell. Side-on anchors are found when apical stress fibers lie parallel to the 
junction. (B) Using head-on and side-on configurations, highlighted in pink, the apical stress fiber, represented by repeated and aligned synaptopodin densities, circled in blue, traces along the junction to form a ring that is structurally and mechanically part of the apical junction. Left panel shows the apical junction of the cell and the right panel shows the bottom of the cell; $X-Y, Y-Z$, and $X-Z$ views are shown. Orange arrowheads mark synaptopodin densities of apical stress fibers in $X-Z$ and $Y-Z$ views. Red arrowheads mark synaptopodin densities of basal stress fibers in $Y-Z$ view. The periodic spacing of synaptopodin densities are similar in apical and basal stress fibers, both have the alternating type I actomyosin organization. (C) Disintegration of periodic synaptopodin organization and formation of type II actomyosin structure, the contractomere, at the apical junction of mature junction. Left and middle panels show the very top and apical junction of the cell, and the right panel shows the bottom of the cell; $X-Y, Y-Z$, and $X-Z$ views are shown. Basal stress fibers remain intact despite disassembly of apical stress fibers.

Figure 4. Three actomyosin structures failed to form in synaptopodin knockdown cells. (A) Apical stress fibers are missing in synaptopodin knockdown cells. (B) Myosin IIB is absent from the apical junction in synaptopodin knockdown cells. Graph shows intensity measurement of myosin IIB along the linear junction. Bars mark the means. (C) Correlation between synaptopodin and myosin IIB levels at the apical junction. (D) Basal stress fibers are missing in synaptopodin knockdown cells. (E) Contractomeres is present in mature MDCK monolayer. White boxes are enlarged to locate the contractomeres, white circles. (F) Contractomeres are missing in synaptopodin knockdown cells. (G) Contractomeric myosin IIB is abolished in synaptopodin knockdown cells. $(\mathrm{H})$ Total cellular levels of myosin IIB and phospho-myosin light chain are lower in synaptopodin knockdown cells. Lane 1 and 3 are whole cell lysate of MDCK parental cells. Lane 2 is whole cell lysate of synaptopodin knockdown cells. Markers are 150, 100, 75, 25 kD.

Figure 5. Tension homeostasis is compromised in synaptopodin knockdown cells. (A) Cartoon showing the balance between cellular contractility and junctional tension. (B) Cartoon illustrating how hydraulic force is used to activate junction contractility. (C) Synaptopodin knockdown increases cell extrusion and decreases alpha-actinin-4 recruitment under force. Red asterisks mark extruded cells. Graph shows increased cell extrusion in synaptopodin knockdown monolayer. Bars mark the means.

Figure 6. Contractomeric myosin II activity is linked to actin accumulation. (A) Inhibition of myosin II ATPase by blebbistatin abolished latrunculin (LatB)-resistant actin at the apical junctional complex. (B) Synaptopodin marks latrunculin-resistant pool of actin at the apical junction. (C) Inhibition of myosin II ATPase by blebbistatin (Bleb) decreased latrunculin-resistant actin accumulation in the presence of barbed-end capping by cytochalasin D (Cyto D). Graphs show intensity measurement of actin on individual contractomeres. Bars mark the means. (D) Non-invasion wound healing assay showing the formation of latrunculin-resistant actin at wound edge, circled, which is inhibited by blebbistatin (Bleb). Latrunculin-resistant actin is formed after 1-hour incubation with cytochalasin D (Cyto D) and latrunculin B (Lat B). In the absence of blebbistatin (Bleb), 
cytochalasin D (Cyto D) or latrunculin B (Lat B), the wound is closed in an hour. Left panels show phase-contrast images of MDCK dome before wounding.

\section{Figure 7. Actin polymerization and myosin II motility are coupled at the}

contractomere. (A) Contractomeres, in circles, are marked by myosin IIB, actin, and alpha-actinin-4, an essential factor for actin polymerization at the junction. Images are immunofluorescence of mature MDCK cell monolayer. (B) Contractomeres in purified membranes, arrows, are marked by myosin IIB, synaptopodin, and alpha-actinin-4. Images are immunofluorescence of purified junction-enriched membranes. (C) Inhibition of actin assembly and membrane contraction by blebbistatin in ex vivo junctional membrane. In vitro actin assembly assays was performed on purified junction-enriched membranes using rhodamine-labelled red actin monomers in the absence or presence of blebbistatin. Frames from time-lapse movies of actin polymerization at the contractomeres are shown. (D) Contractomeric myosin IIB marks the site of actin assembly on purified membrane. Immunofluorescence for myosin IIB were performed after the actin assembly reaction on purified membrane. (E) Negative-stain electron microscopy showing actin filaments inserted at large electron-dense contractomeres. Contractomeres lack the characteristic bipolar myosin minifilaments but can be mechanically and chemically dissociated into sub-complexes, including a sub-complex containing myosin II monomer. The 2 motor heads of the myosin II monomer interact with actin filament (yellow arrows) and the tail region of the myosin II monomer interacts with electron-dense materials (red arrows). (F) Protocol for reconstitution actin assembly assay using stripped membranes and recombinant alpha-actinin-4 full-length or truncated proteins. Bottom panels shows that alpha-actinin-4 with one actin-binding domain failed to crosslink Alexa 647-labelled actin filaments into bundles that can be readily visualized under the light microscope. (G) Actin assembly on the contractomere requires interaction of alpha-actinin- 4 with actin. Reconstitution actin assembly assay showing alpha-actinin-4 lacking the actin-binding domains was recruited to the contractomere but failed to support actin assembly. Graph shows intensity measurement of Oregon green-labelled alpha-actinin-4 on individual contractomeres; boxes represent 75 percentile and error bars are standard deviation. $(H)$ Contractomeric actin assembly requires only one actin-binding domain of alpha-actinin-4. Graph shows intensity measurement of rhodamine-labelled actin on individual contractomeres. Bars mark the means.

Figure 8. Contractomere glides on the membrane to shorten or lengthen the junction. (A) Contractomere (blue circles) glides towards each other to constrict the apical junction during apoptotic cell extrusion (orange asterisk). Frames from time-lapse wide-field movie of venus-alpha-actinin-1. Yellow arrows mark the paths of contractomere movement. Orange circle shows the location of contractomeres after constriction. (B) Contractomere (yellow circles) glides toward each other to constrict the apical junction during live-cell extrusion (white asterisk). Frames from time-lapse wide-field movie of venus-alpha-actinin-1. Live-cell extrusion is not associated with membrane blebbing of extruding cell. Yellow arrows mark the paths of contractomere movement. Orange circle shows the location of contractomeres after constriction. (C) Contractomere (circles) glides towards each other to constrict the apical junction during apoptotic cell extrusion (white 
asterisk). Frames from time-lapse structured-illumination movies of venus-alpha-actinin-1. Orange circle show the location of contractomeres after completion of constriction. (D) Contractomere (circles) glides towards each other to constrict the apical junction during live-cell extrusion (white asterisk). Frames from time-lapse movies of structuredillumination microscopy of venus-alpha-actinin-1. Live-cell extrusion is not associated with membrane blebbing of extruding cell. (E) Contractomeres (circles) glide to shorten the apical junction surround the extruding cell (white asterisk) while lengthen the apical junction in the neighboring cells (blue and orange arrows). Frames from time-lapse structured-illumination movies of venus-alpha-actinin-1. Live-cell extrusion is not associated with membrane blebbing of extruding cell. (F) Contractomere slides during intercellular movement. Sliding of contractomere can either shorten or lengthen the junction. Cells \#1 and \#2 are used to illustrate that some junctions increased but the others decreased their length, resulting in almost zero net change in total junctional length. Frames from time-lapse structured-illumination movies of venus-alpha-actinin-1. (G) Gliding of contractomeres to reproportion junctional lengths. Movement of contractomere $\mathrm{b}$ towards contractomere a resulted in shortening the junction between $\mathrm{a}$ and $\mathrm{b}$ with concomitant lengthening of the junction between contractomeres $b$ and $c$. Frames from time-lapse wide-field movies of venus-alpha-actinin-1. $(\mathrm{H})$ Gliding of contractomeres to reproportion junctional lengths. Movement of contractomere $b$ away from contractomere $a$ resulted in lengthening of the junction between $a$ and $b$ with concomitant shortening of the junction between contractomeres $b$ and $c$. Frames from time-lapse wide-field movie of synaptopodin-venus.

\section{Figure 9. Contractomere and apical junction is under compressive force as} measured by a new alpha-actinin-4 FRET tension sensor. (A) Four alpha-actinin FRET-based sensors with the force-sensing cassette, sstFRET, inserted at different position within or at the end of the alpha-actinin proteins. When the force sensor is located at the C-terminus, alpha-actinin-1-sstFRET-C, no force can be applied to the sensor and the measured FRET index of 0.65 is the baseline of the sstFRET cassette. Alpha-actinin1-M-sstFRET reported a tensile force at the junction whereas alpha-actinin-4-sstFRET408 and alpha-actinin-4-sstFRET522 reported compressive forces. (B) Lack of FRET difference between protrusive cell edge and contractile cell junction using alpha-actinin-1sstFRET-C. (C) Lack of FRET difference between protrusive cell edge and contractile cell junction using alpha-actinin-1-M-sstFRET. (D) FRET difference between protrusive cell edge and contractile cell junction using alpha-actinin-4-sstFRET522. (E) Increased compressive force at linear junction and contractomere in maturing epithelial monolayer. (F) Junction and contractomere contractility could compress alpha-actinin-associated structures at the junction.

Figure 10. Synaptopodin is responsible for 2 new actomyosin structures at the apical junction to support extrinsic and intrinsic contractility. (A) Comparison of actomyosin meshwork and cables with apical stress fiber, type I, and contractomere, type II, actomyosin organizations. Apical stress fibers can selectively link neighboring junctions as well as junctions from opposite sides of a cell. Contractomere is a unique actomyosin structure that contains non-filamentous myosin II. (B) Comparison between actomyosin 
meshwork and apical stress fibers is shown at the top panels. Apical stress fibers inserted at cell-cell adhesion can propagate force and generate tissue-level tension over many cells in an epithelial monolayer, the bottom panel. (C) Shortening and lengthening the junction by "walking" the contractomere. Motility of contractomeres contributes to apical junction constriction during cell extrusion and pure-string wound closure. The prevailing model of junction remodeling requires disassembly of the actomyosin cortex and endocytosis of existing junction to shorten a junction. (D) Contractomere is directly powered by myosin II and actin polymerization to generate force locally at the junction while apical stress fiber powered by myosin II are at a distance away from the junction. Contractomeres generate intrinsic force whereas apical stress fibers or actomyosin meshworks generate extrinsic force with respect to the junction. (E) Key to cartoon. 


\section{Supplementary Figure Legend}

Figure S1. MDCK cell expresses synaptopodin isoform A. (A) Synaptopodin is encoded by 3 exons, resulting in 4 splice variants. We have raised antibodies to each spliced region to characterize the expression of synaptopodin isoforms in different mammalian tissues and cells. Table summarized results from western blot studies using our newly generated antibodies (see methods section). (B) Western blot using antibodies against region encoded by exon 2, thus recognizing all synaptopodin isoforms.

Figure S2. Synaptopodin is localized to basal stress fibers. (A) Alternating arrangement of synaptopodin and myosin IIB forming basal stress fiber sarcomeric-like repeats. (B) Synaptopodin-associated basal stress fibers are inserted into vinculin-decorated focal adhesions.

Figure S3. Myosin IIA is localized to apical and basal stress fibers in MDCK cells. (A) Periodic placement of myosin IIA at the apical stress fiber. (B) Periodic placement of myosin IIA of basal stress fibers.

Figure S4. Polarized phenotype of MDCK cells are unchanged by synaptopodin depletion. Reconstruction of Z-stacks shows that synaptopodin knockdown cells have similar overall morphology as parental MDCK cells.

Figure S5. Myosin IIA is present at the apical junction in synaptopodin knockdown monolayers. (A) Junctional myosin IIA level is unchanged in synaptopodin knockdown cells. (B) Myosin IIA basal stress fibers are converted to myosin IIA meshworks in synaptopodin knockdown cells.

Figure S6. Myosin IIB is absent from the apical junction in synaptopodin knockdown cells despite the presence of actin. Synaptopodin knockdowns electively affects myosin IIB but not actin in mature MDCK monolayer.

Figure S7. Myosin IIB marks the sites of actin assembly on purified junction-enriched membranes. Immunofluorescence staining for myosin IIB was performed on junctional membranes after the actin assembly assay with rhodamine green-actin.

Figure S8. Contractomeres reposition during intercellular movement resulting in conservation of overall junctional length. (A) time-lapse from live-cell structured illumination microscopy of alpha-actinin-venus showing contractomere at the beginning (pink circles) and at the end (yellow circles) of a 4-hour movie. During intercellular organization, junctional length can shorten when 2 contractomeres move towards each other and junctional length can extend when 2 contractomeres move away from each other. (B) Plating of MDCK cells on collagen I at confluent density results in intercellular movement and neighbor exchange. Total junctional length of individual cell remained constant. 
bioRxiv preprint doi: https://doi.org/10.1101/2020.12.30.424702; this version posted January 1,2021 . The copyright holder for this preprint (which was not certified by peer review) is the author/funder, who has granted bioRxiv a license to display the preprint in perpetuity. It is made available under aCC-BY-NC-ND 4.0 International license. 


\section{Movie Legend}

Movie 1. Live-cell time-lapse of venus-synaptopodin showing apical stress fiber floating in apical cytoplasm.

Movie 2. Actin assembly assay using rhodamine-actin and purified junctional membrane showing the coupling of membrane contraction to actin assembly. Frame rate is $12 \mathrm{~min}$ real time per second of movie.

Movie 3. Actin assembly assay in the presence of blebbistatin using rhodamine-actin and purified junctional membrane showing inhibition of membrane contraction and actin assembly. Frame rate is 12 min real time per second of movie.

Movie 4. Live-cell time-lapse of venus-alpha-actinin-1 showing contractomere movement during apoptotic cell extrusion.

Movie 5. Live-cell time-lapse of venus-alpha-actinin-1 showing contractomere movement during live-cell extrusion.

Movie 6. Structured-illumination live-cell time-lapse of venus-alpha-actinin-1 showing contractomere movement during apoptotic cell extrusion.

Movie 7. Structured-illumination live-cell time-lapse of venus-alpha-actinin-1 showing contractomere movement during live-cell extrusion.

Movie 8. Live-cell time-lapse of venus-alpha-actinin-1 showing contractomere movement during apical junction constriction.

Movie 9. Structured-illumination live-cell time-lapse of venus-alpha-actinin-1 showing contractomere movement during intercellular movement. Purple circles mark contractomeres at the beginning of movie. Yellow circles mark contractomeres at the end of movie.

Movie 10. Live-cell time-lapse of venus-alpha-actinin-1 showing neighbor exchange during intercellular movement.

Movie 11. Live-cell time-lapse of venus-alpha-actinin-1 showing 2 oscillating contractomeres back-and-forth on the junction in mature monolayer.

Movie 12. Live-cell time-lapse of venus-alpha-actinin-1 showing contractomeres in mature monolayer.

Movie 13. Live-cell time-lapse of venus-alpha-actinin-1 showing oscillating contractomeres back-and-forth along the linear junctions in mature monolayer. 
bioRxiv preprint doi: https://doi.org/10.1101/2020.12.30.424702; this version posted January 1,2021. The copyright holder for this preprint

(which was not certified by peer review) is the author/funder, who has granted bioRxiv a license to display the preprint in perpetuity. It is made available under aCC-BY-NC-ND 4.0 International license.

Movie 14. Live-cell time-lapse of venus-synaptopodin showing 2 oscillating contractomeres back-and-forth on the junction in mature monolayer.

Movie 15. Live-cell time-lapse of venus-synaptopodin showing oscillating contractomeres back-and-forth along the linear junctions in mature monolayer. 


\section{References}

Abreu-Blanco, M.T., J.M. Verboon, R. Liu, J.J. Watts, and S.M. Parkhurst. 2012. Drosophila embryos close epithelial wounds using a combination of cellular protrusions and an actomyosin purse string. J Cell Sci. 125:5984-5997.

Amano, M., M. Ito, K. Kimura, Y. Fukata, K. Chihara, T. Nakano, Y. Matsuura, and K. Kaibuchi. 1996. Phosphorylation and activation of myosin by Rho-associated kinase (Rho-kinase). J Biol Chem. 271:20246-20249.

Aoki, R., T. Kitaguchi, M. Oya, Y. Yanagihara, M. Sato, A. Miyawaki, and T. Tsuboi. 2010. Duration of fusion pore opening and the amount of hormone released are regulated by myosin II during kiss-and-run exocytosis. Biochem J. 429:497-504.

Arnold, T.R., J.H. Shawky, R.E. Stephenson, K.M. Dinshaw, T. Higashi, F. Huq, L.A. Davidson, and A.L. Miller. 2019. Anillin regulates epithelial cell mechanics by structuring the medial-apical actomyosin network. Elife. 8.

Asanuma, K., K. Kim, J. Oh, L. Giardino, S. Chabanis, C. Faul, J. Reiser, and P. Mundel. 2005. Synaptopodin regulates the actin-bundling activity of alpha-actinin in an isoform-specific manner. J Clin Invest. 115:1188-1198.

Badirou, I., J. Pan, C. Legrand, A. Wang, L. Lordier, S. Boukour, A. Roy, W. Vainchenker, and Y. Chang. 2014. Carboxyl-terminal-dependent recruitment of nonmuscle myosin II to megakaryocyte contractile ring during polyploidization. Blood. 124:2564-2568.

Beach, J.R., and T.T. Egelhoff. 2009. Myosin II recruitment during cytokinesis independent of centralspindlin-mediated phosphorylation. J Biol Chem. 284:27377-27383.

Bement, W.M., P. Forscher, and M.S. Mooseker. 1993. A novel cytoskeletal structure involved in purse string wound closure and cell polarity maintenance. J Cell Biol. 121:565-578.

Bresnick, A.R. 1999. Molecular mechanisms of nonmuscle myosin-Il regulation. Curr Opin Cell Biol. 11:26-33.

Brieher, W.M., M. Coughlin, and T.J. Mitchison. 2004. Fascin-mediated propulsion of Listeria monocytogenes independent of frequent nucleation by the Arp2/3 complex. J Cell Biol. 165:233-242.

Brieher, W.M., H.Y. Kueh, B.A. Ballif, and T.J. Mitchison. 2006. Rapid actin monomer-insensitive depolymerization of Listeria actin comet tails by cofilin, coronin, and Aip1. J Cell Biol. 175:315-324.

Cavanaugh, K.E., M.F. Staddon, E. Munro, S. Banerjee, and M.L. Gardel. 2020. RhoA Mediates Epithelial Cell Shape Changes via Mechanosensitive Endocytosis. Dev Cell. 52:152-166 e155.

Chang, C.W., and S. Kumar. 2015. Differential Contributions of Nonmuscle Myosin II Isoforms and Functional Domains to Stress Fiber Mechanics. Sci Rep. 5:13736.

Clark, A.G., A.L. Miller, E. Vaughan, H.Y. Yu, R. Penkert, and W.M. Bement. 2009. Integration of single and multicellular wound responses. Curr Biol. 19:1389-1395.

Danjo, Y., and I.K. Gipson. 1998. Actin 'purse string' filaments are anchored by E-cadherinmediated adherens junctions at the leading edge of the epithelial wound, providing coordinated cell movement. J Cell Sci. 111 ( Pt 22):3323-3332.

Dorfel, M.J., J.K. Westphal, C. Bellmann, S.M. Krug, J. Cording, S. Mittag, R. Tauber, M. Fromm, I.E. Blasig, and O. Huber. 2013. CK2-dependent phosphorylation of occludin regulates the interaction with ZO-proteins and tight junction integrity. Cell Commun Signal. 11:40. 
Dulyaninova, N.G., and A.R. Bresnick. 2013. The heavy chain has its day: regulation of myosin-II assembly. Bioarchitecture. 3:77-85.

Eckert, J.J., A. McCallum, A. Mears, M.G. Rumsby, I.T. Cameron, and T.P. Fleming. 2005. Relative contribution of cell contact pattern, specific PKC isoforms and gap junctional communication in tight junction assembly in the mouse early embryo. Dev Biol. 288:234247.

Eisenhoffer, G.T., P.D. Loftus, M. Yoshigi, H. Otsuna, C.B. Chien, P.A. Morcos, and J. Rosenblatt. 2012. Crowding induces live cell extrusion to maintain homeostatic cell numbers in epithelia. Nature. 484:546-549.

Even-Faitelson, L., and S. Ravid. 2006. PAK1 and aPKCzeta regulate myosin II-B phosphorylation: a novel signaling pathway regulating filament assembly. Mol Biol Cell. 17:2869-2881.

Fanning, A.S., C.M. Van Itallie, and J.M. Anderson. 2012. Zonula occludens-1 and -2 regulate apical cell structure and the zonula adherens cytoskeleton in polarized epithelia. Mol Biol Cell. 23:577-590.

Florian, P., T. Schoneberg, J.D. Schulzke, M. Fromm, and A.H. Gitter. 2002. Single-cell epithelial defects close rapidly by an actinomyosin purse string mechanism with functional tight junctions. J Physiol. 545:485-499.

Franco, J.J., Y. Atieh, C.D. Bryan, K.M. Kwan, and G.T. Eisenhoffer. 2019. Cellular crowding influences extrusion and proliferation to facilitate epithelial tissue repair. Mol Biol Cell. 30:1890-1899.

Ganguly, C., I.C. Baines, E.D. Korn, and J. Sellers. 1992. Regulation of the actin-activated ATPase and in vitro motility activities of monomeric and filamentous Acanthamoeba myosin II. J Biol Chem. 267:20900-20904.

Gomez, G.A., R.W. McLachlan, and A.S. Yap. 2011. Productive tension: force-sensing and homeostasis of cell-cell junctions. Trends Cell Biol. 21:499-505.

Helfrich, I., A. Schmitz, P. Zigrino, C. Michels, I. Haase, A. le Bivic, M. Leitges, and C.M. Niessen. 2007. Role of aPKC isoforms and their binding partners Par3 and Par6 in epidermal barrier formation. J Invest Dermatol. 127:782-791.

Heuze, M.L., G.H.N. Sankara Narayana, J. D'Alessandro, V. Cellerin, T. Dang, D.S. Williams, J.C. Van Hest, P. Marcq, R.M. Mege, and B. Ladoux. 2019. Myosin II isoforms play distinct roles in adherens junction biogenesis. Elife. 8.

Juanes-Garcia, A., J.R. Chapman, R. Aguilar-Cuenca, C. Delgado-Arevalo, J. Hodges, L.A. Whitmore, J. Shabanowitz, D.F. Hunt, A.R. Horwitz, and M. Vicente-Manzanares. 2015. A regulatory motif in nonmuscle myosin II-B regulates its role in migratory front-back polarity. J Cell Biol. 209:23-32.

Kannan, N., and V.W. Tang. 2015. Synaptopodin couples epithelial contractility to alpha-actinin-4dependent junction maturation. J Cell Biol. 211:407-434.

Kannan, N., and V.W. Tang. 2018. Myosin-1c promotes E-cadherin tension and force-dependent recruitment of alpha-actinin to the epithelial cell junction. J Cell Sci. 131.

Kassianidou, E., J.H. Hughes, and S. Kumar. 2017. Activation of ROCK and MLCK tunes regional stress fiber formation and mechanics via preferential myosin light chain phosphorylation. Mol Biol Cell. 28:3832-3843.

Kiboku, T., T. Katoh, A. Nakamura, A. Kitamura, M. Kinjo, Y. Murakami, and M. Takahashi. 2013. Nonmuscle myosin II folds into a $10 \mathrm{~S}$ form via two portions of tail for dynamic subcellular localization. Genes Cells. 18:90-109. 
Kremerskothen, J., C. Plaas, S. Kindler, M. Frotscher, and A. Barnekow. 2005. Synaptopodin, a molecule involved in the formation of the dendritic spine apparatus, is a dual actin/alphaactinin binding protein. J Neurochem. 92:597-606.

Kuipers, D., A. Mehonic, M. Kajita, L. Peter, Y. Fujita, T. Duke, G. Charras, and J.E. Gale. 2014. Epithelial repair is a two-stage process driven first by dying cells and then by their neighbours. J Cell Sci. 127:1229-1241.

Kuragano, M., T.Q.P. Uyeda, K. Kamijo, Y. Murakami, and M. Takahashi. 2018. Different contributions of nonmuscle myosin IIA and IIB to the organization of stress fiber subtypes in fibroblasts. Mol Biol Cell. 29:911-922.

le Duc, Q., Q. Shi, I. Blonk, A. Sonnenberg, N. Wang, D. Leckband, and J. de Rooij. 2010. Vinculin potentiates E-cadherin mechanosensing and is recruited to actin-anchored sites within adherens junctions in a myosin II-dependent manner. J Cell Biol. 189:1107-1115.

Li, J.X.H., V.W. Tang, and W.M. Brieher. 2020. Actin protrusions push at apical junctions to maintain E-cadherin adhesion. Proc Natl Acad Sci U S A. 117:432-438.

Limouze, J., A.F. Straight, T. Mitchison, and J.R. Sellers. 2004. Specificity of blebbistatin, an inhibitor of myosin II. J Muscle Res Cell Motil. 25:337-341.

Liu, J., D.W. Taylor, and K.A. Taylor. 2004. A 3-D reconstruction of smooth muscle alpha-actinin by CryoEm reveals two different conformations at the actin-binding region. $J$ Mol Biol. 338:115-125.

Liu, X., N. Billington, S. Shu, S.H. Yu, G. Piszczek, J.R. Sellers, and E.D. Korn. 2017. Effect of ATP and regulatory light-chain phosphorylation on the polymerization of mammalian nonmuscle myosin II. Proc Natl Acad Sci U S A. 114:E6516-E6525.

Liu, X., S. Shu, N. Billington, C.D. Williamson, S. Yu, H. Brzeska, J.G. Donaldson, J.R. Sellers, and E.D. Korn. 2016. Mammalian Nonmuscle Myosin II Binds to Anionic Phospholipids with Concomitant Dissociation of the Regulatory Light Chain. J Biol Chem. 291:24828-24837.

Liu, X., S. Shu, and E.D. Korn. 2018. Polymerization pathway of mammalian nonmuscle myosin $2 \mathrm{~s}$. Proc Natl Acad Sci U S A. 115:E7101-E7108.

Melli, L., N. Billington, S.A. Sun, J.E. Bird, A. Nagy, T.B. Friedman, Y. Takagi, and J.R. Sellers. 2018. Bipolar filaments of human nonmuscle myosin 2-A and 2-B have distinct motile and mechanical properties. Elife. 7.

Meng, F., and F. Sachs. 2011. Visualizing dynamic cytoplasmic forces with a compliance-matched FRET sensor. J Cell Sci. 124:261-269.

Meng, F., and F. Sachs. 2012. Orientation-based FRET sensor for real-time imaging of cellular forces. J Cell Sci. 125:743-750.

Meyer, R.K., and U. Aebi. 1990. Bundling of actin filaments by alpha-actinin depends on its molecular length. J Cell Biol. 110:2013-2024.

Milberg, O., A. Shitara, S. Ebrahim, A. Masedunskas, M. Tora, D.T. Tran, Y. Chen, M.A. Conti, R.S. Adelstein, K.G. Ten Hagen, and R. Weigert. 2017. Concerted actions of distinct nonmuscle myosin II isoforms drive intracellular membrane remodeling in live animals. J Cell Biol. 216:1925-1936.

Morin, T.R., Jr., S.A. Ghassem-Zadeh, and J. Lee. 2014. Traction force microscopy in rapidly moving cells reveals separate roles for ROCK and MLCK in the mechanics of retraction. Exp Cell Res. 326:280-294.

Murakami, N., V.P. Chauhan, and M. Elzinga. 1998. Two nonmuscle myosin II heavy chain isoforms expressed in rabbit brains: filament forming properties, the effects of phosphorylation by 
protein kinase $\mathrm{C}$ and casein kinase II, and location of the phosphorylation sites.

Biochemistry. 37:1989-2003.

Murakami, N., S. Matsumura, and A. Kumon. 1984. Purification and identification of myosin heavy chain kinase from bovine brain. J Biochem. 95:651-660.

Nanavati, B.N., A.S. Yap, and J.L. Teo. 2020. Symmetry Breaking and Epithelial Cell Extrusion. Cells. 9.

Naumanen, P., P. Lappalainen, and P. Hotulainen. 2008. Mechanisms of actin stress fibre assembly. J Microsc. 231:446-454.

Norstrom, M.F., P.A. Smithback, and R.S. Rock. 2010. Unconventional processive mechanics of non-muscle myosin IIB. J Biol Chem. 285:26326-26334.

Pellegrin, S., and H. Mellor. 2007. Actin stress fibres. J Cell Sci. 120:3491-3499.

Pinheiro, D., and Y. Bellaiche. 2018. Mechanical Force-Driven Adherens Junction Remodeling and Epithelial Dynamics. Dev Cell. 47:3-19.

Raleigh, D.R., D.M. Boe, D. Yu, C.R. Weber, A.M. Marchiando, E.M. Bradford, Y. Wang, L. Wu, E.E. Schneeberger, L. Shen, and J.R. Turner. 2011. Occludin S408 phosphorylation regulates tight junction protein interactions and barrier function. J Cell Biol. 193:565-582.

Ramamurthy, B., C.M. Yengo, A.F. Straight, T.J. Mitchison, and H.L. Sweeney. 2004. Kinetic mechanism of blebbistatin inhibition of nonmuscle myosin Ilb. Biochemistry. 43:1483214839.

Roper, K. 2015. Integration of cell-cell adhesion and contractile actomyosin activity during morphogenesis. Curr Top Dev Biol. 112:103-127.

Rosenberg, M., R. Straussman, A. Ben-Ya'acov, D. Ronen, and S. Ravid. 2008. MHC-IIB filament assembly and cellular localization are governed by the rod net charge. PLoS One. 3:e1496.

Roy, A., L. Lordier, S. Mazzi, Y. Chang, V. Lapierre, J. Larghero, N. Debili, H. Raslova, and W. Vainchenker. 2016. Activity of nonmuscle myosin II isoforms determines localization at the cleavage furrow of megakaryocytes. Blood. 128:3137-3145.

Sandquist, J.C., K.I. Swenson, K.A. Demali, K. Burridge, and A.R. Means. 2006. Rho kinase differentially regulates phosphorylation of nonmuscle myosin II isoforms $A$ and $B$ during cell rounding and migration. J Biol Chem. 281:35873-35883.

Saw, T.B., A. Doostmohammadi, V. Nier, L. Kocgozlu, S. Thampi, Y. Toyama, P. Marcq, C.T. Lim, J.M. Yeomans, and B. Ladoux. 2017. Topological defects in epithelia govern cell death and extrusion. Nature. 544:212-216.

Shindo, A., and J.B. Wallingford. 2014. PCP and septins compartmentalize cortical actomyosin to direct collective cell movement. Science. 343:649-652.

Shutova, M.S., S.B. Asokan, S. Talwar, R.K. Assoian, J.E. Bear, and T.M. Svitkina. 2017. Self-sorting of nonmuscle myosins IIA and IIB polarizes the cytoskeleton and modulates cell motility. $J$ Cell Biol. 216:2877-2889.

Shutova, M.S., W.A. Spessott, C.G. Giraudo, and T. Svitkina. 2014. Endogenous species of mammalian nonmuscle myosin IIA and IIB include activated monomers and heteropolymers. Curr Biol. 24:1958-1968.

Smutny, M., H.L. Cox, J.M. Leerberg, E.M. Kovacs, M.A. Conti, C. Ferguson, N.A. Hamilton, R.G. Parton, R.S. Adelstein, and A.S. Yap. 2010. Myosin II isoforms identify distinct functional modules that support integrity of the epithelial zonula adherens. Nat Cell Biol. 12:696-702.

Stam, S., J. Alberts, M.L. Gardel, and E. Munro. 2015. Isoforms Confer Characteristic Force Generation and Mechanosensation by Myosin II Filaments. Biophys J. 108:1997-2006. 
Tang, V.W., and W.M. Brieher. 2012. alpha-Actinin-4/FSGS1 is required for Arp2/3-dependent actin assembly at the adherens junction. J Cell Biol. 196:115-130.

Tang, V.W., and W.M. Brieher. 2013. FSGS3/CD2AP is a barbed-end capping protein that stabilizes actin and strengthens adherens junctions. J Cell Biol. 203:815-833.

Tang, V.W., and D.A. Goodenough. 2003. Paracellular ion channel at the tight junction. Biophys J. 84:1660-1673.

Totsukawa, G., Y. Wu, Y. Sasaki, D.J. Hartshorne, Y. Yamakita, S. Yamashiro, and F. Matsumura. 2004. Distinct roles of MLCK and ROCK in the regulation of membrane protrusions and focal adhesion dynamics during cell migration of fibroblasts. J Cell Biol. 164:427-439.

Uhlen, M., L. Fagerberg, B.M. Hallstrom, C. Lindskog, P. Oksvold, A. Mardinoglu, A. Sivertsson, C. Kampf, E. Sjostedt, A. Asplund, I. Olsson, K. Edlund, E. Lundberg, S. Navani, C.A. Szigyarto, J. Odeberg, D. Djureinovic, J.O. Takanen, S. Hober, T. Alm, P.H. Edqvist, H. Berling, H. Tegel, J. Mulder, J. Rockberg, P. Nilsson, J.M. Schwenk, M. Hamsten, K. von Feilitzen, M. Forsberg, L. Persson, F. Johansson, M. Zwahlen, G. von Heijne, J. Nielsen, and F. Ponten. 2015. Proteomics. Tissue-based map of the human proteome. Science. 347:1260419.

Verma, D., F. Meng, F. Sachs, and S.Z. Hua. 2015. Flow-induced focal adhesion remodeling mediated by local cytoskeletal stresses and reorganization. Cell Adh Migr. 9:432-440.

Verma, S., S.P. Han, M. Michael, G.A. Gomez, Z. Yang, R.D. Teasdale, A. Ratheesh, E.M. Kovacs, R.G. Ali, and A.S. Yap. 2012. A WAVE2-Arp2/3 actin nucleator apparatus supports junctional tension at the epithelial zonula adherens. Mol Biol Cell. 23:4601-4610.

Vicente-Manzanares, M., X. Ma, R.S. Adelstein, and A.R. Horwitz. 2009. Non-muscle myosin II takes centre stage in cell adhesion and migration. Nat Rev Mol Cell Biol. 10:778-790.

Wang, F., M. Kovacs, A. Hu, J. Limouze, E.V. Harvey, and J.R. Sellers. 2003. Kinetic mechanism of non-muscle myosin IIB: functional adaptations for tension generation and maintenance. $J$ Biol Chem. 278:27439-27448.

Wood, W., A. Jacinto, R. Grose, S. Woolner, J. Gale, C. Wilson, and P. Martin. 2002. Wound healing recapitulates morphogenesis in Drosophila embryos. Nat Cell Biol. 4:907-912.

Wu, S.K., S. Budnar, A.S. Yap, and G.A. Gomez. 2014. Pulsatile contractility of actomyosin networks organizes the cellular cortex at lateral cadherin junctions. Eur J Cell Biol. 93:396-404. 
Apical Junction of

MDCK Monolayer

myosin IIB synaptopodin A-DYK loechst

B

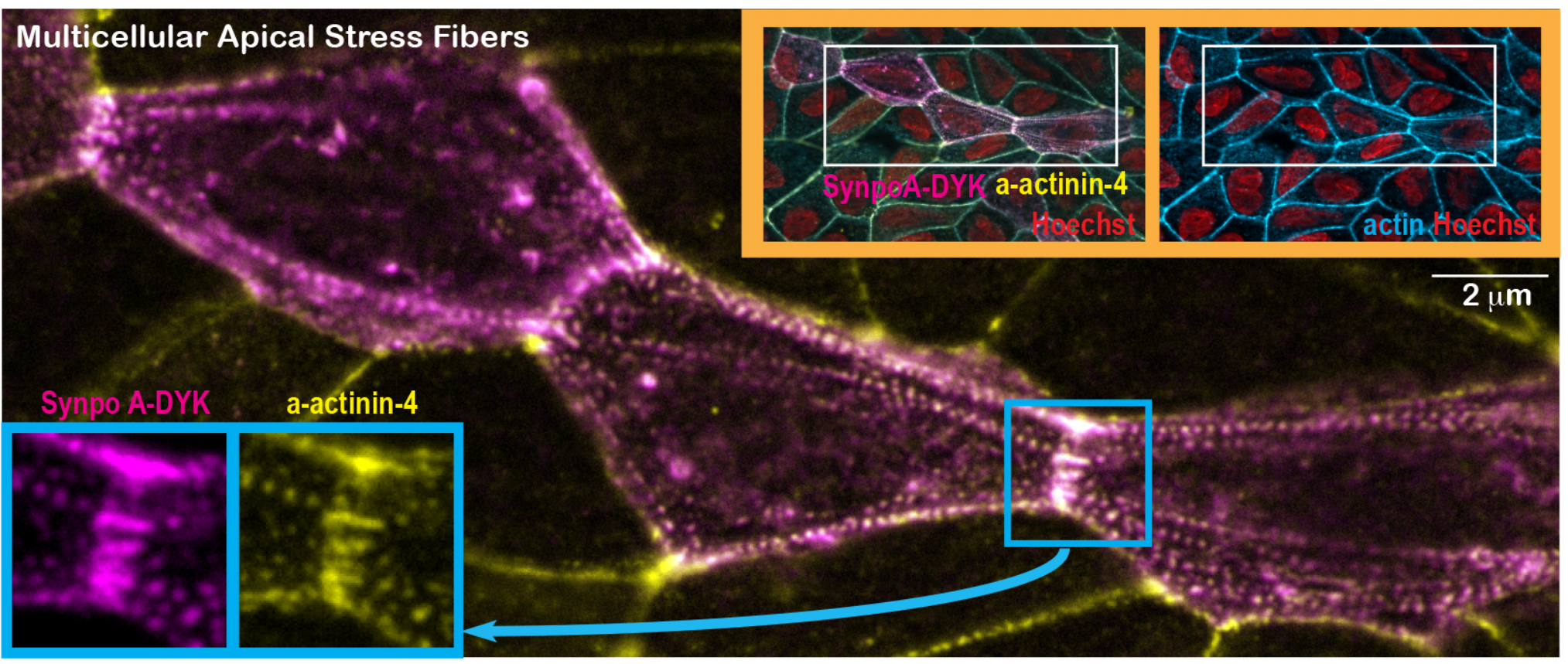

C Two types of myosin II organization in developing MDCK monolayer
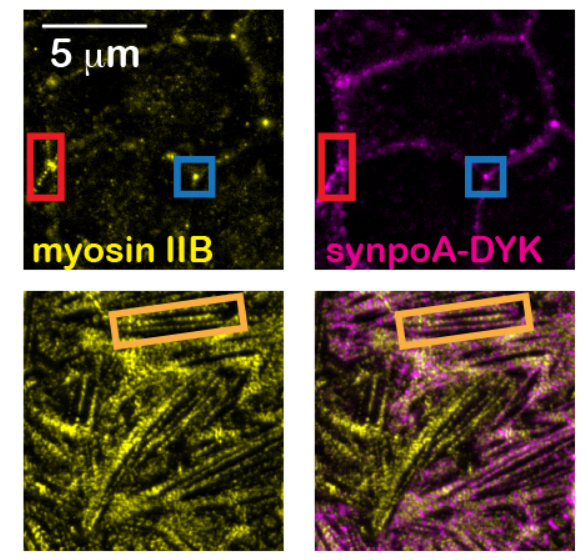
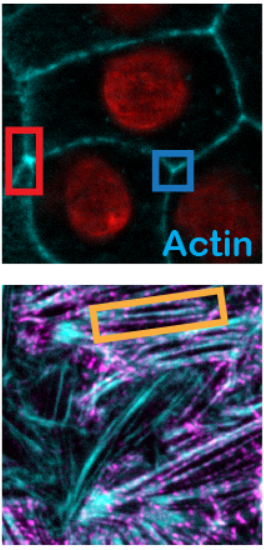

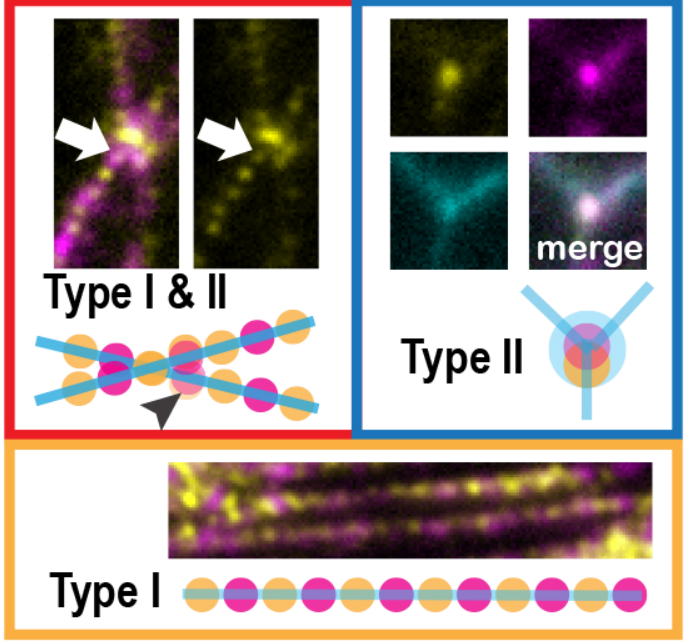

Figure 1 


\section{A Developing} Monolayer

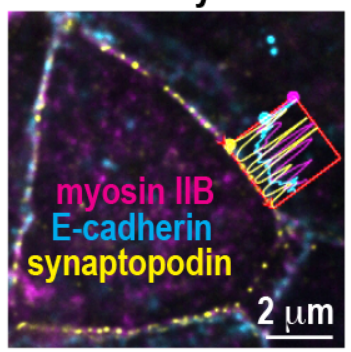

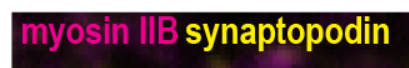

$1 \mu \mathrm{m}$

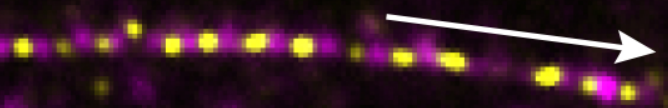

B

Association of apical stress fiber with E-cadherin junction in developing Monolayer

E-cadherin synaptopodin "inyosin IIB

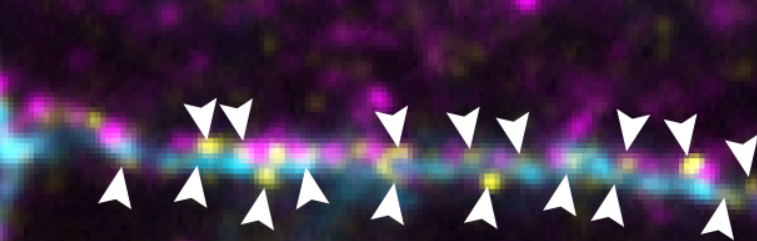

$1 \mu \mathrm{m}$

44
C
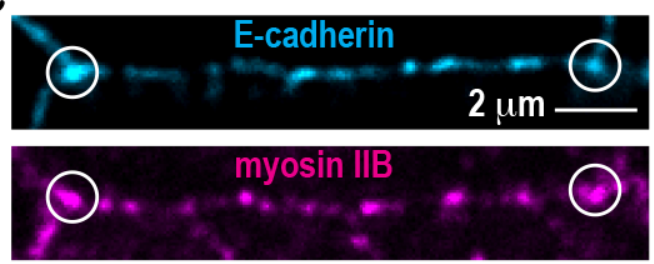

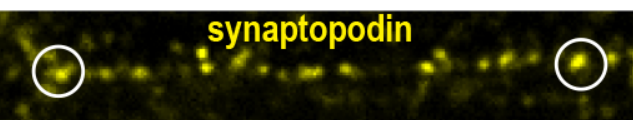

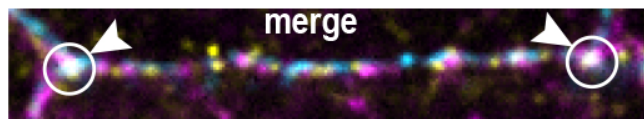

Disassembly of apical stress fibers during transition to mature Monolayer

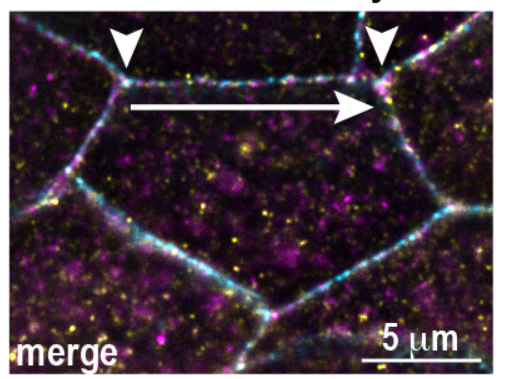

D

Mature Monolayer

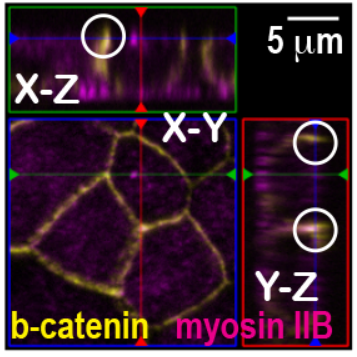

Formation of a junctional complex by alpha-actinin-4, synaptopodin, myosin IIB actin $2 \overline{\mu m}$
Assembly of a complex containing alpha-actinin-4, synaptopodin, myosin IIB
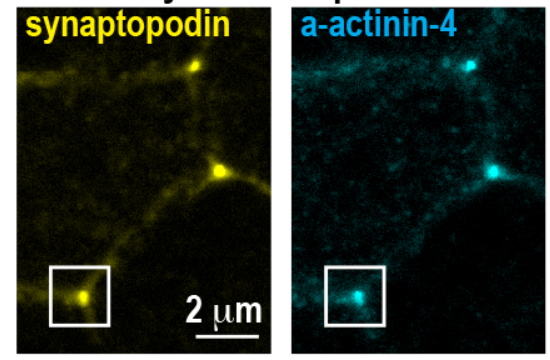

Transitioning to Mature Monolayer

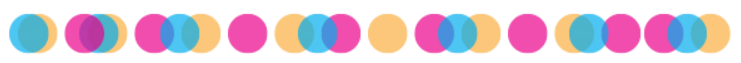
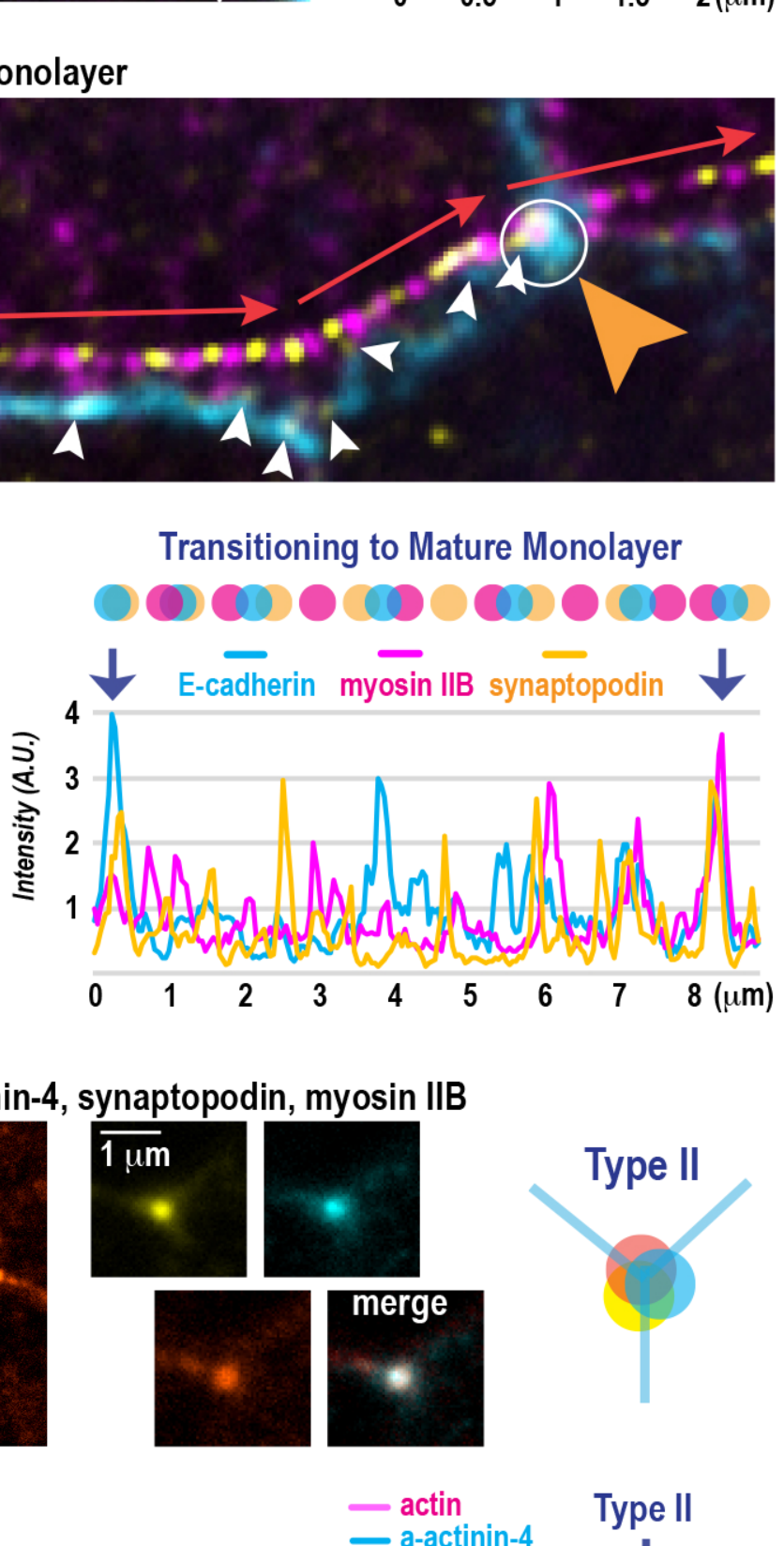

- actin

- E-cadherin - synaptopodin 


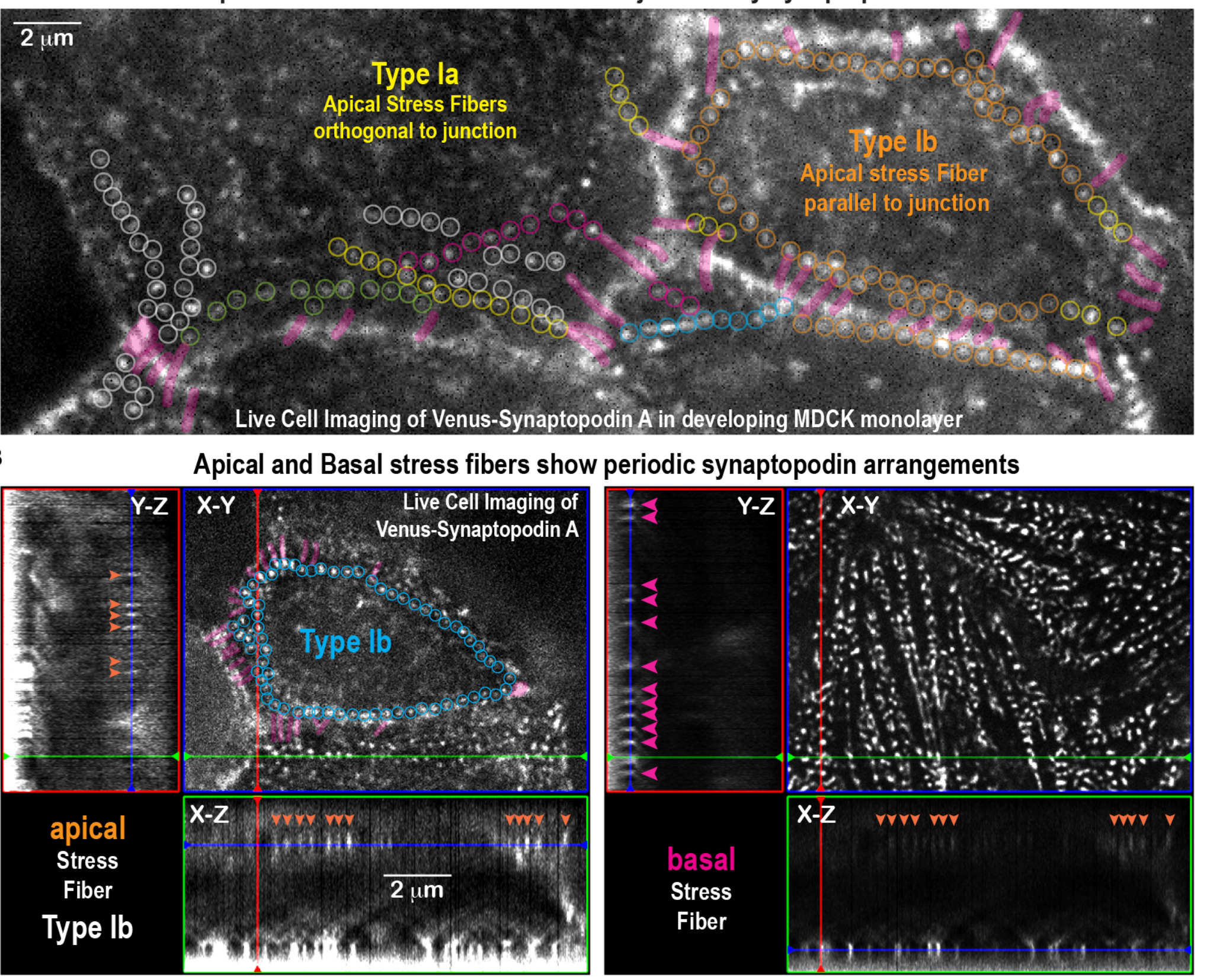

C Disassembly of Apical stress fibers but not Basal stress fibers during Junction Maturation

$X-Y$
Disassembly of Apical stress fibers

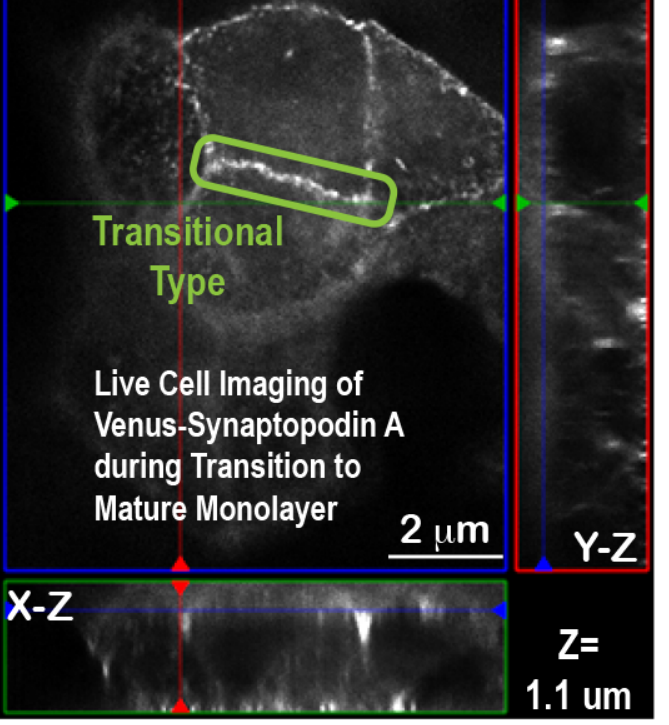

a.

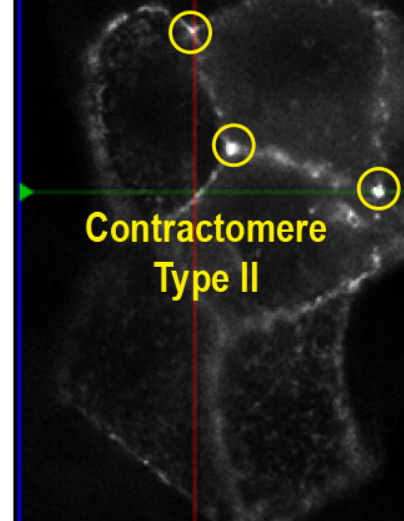

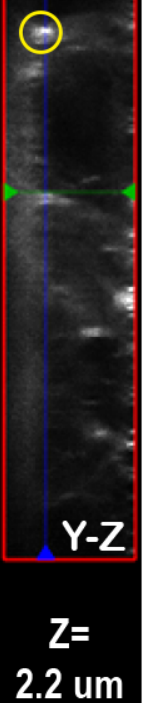

$X-Y$

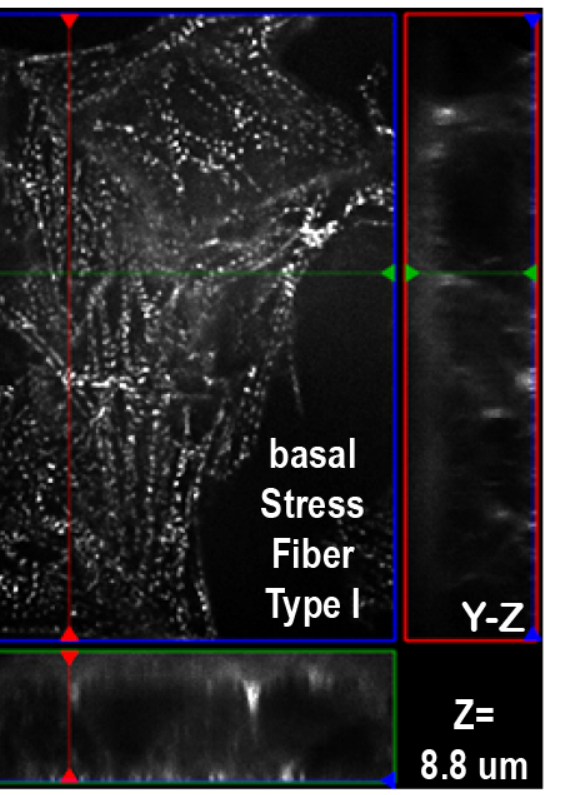


(which was not certified by peer review) is the author/funder, who has granted bioniviv a license to disph

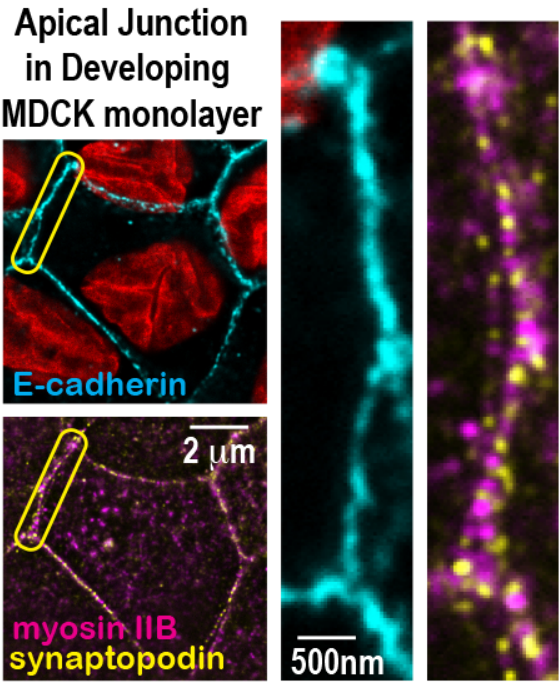

C

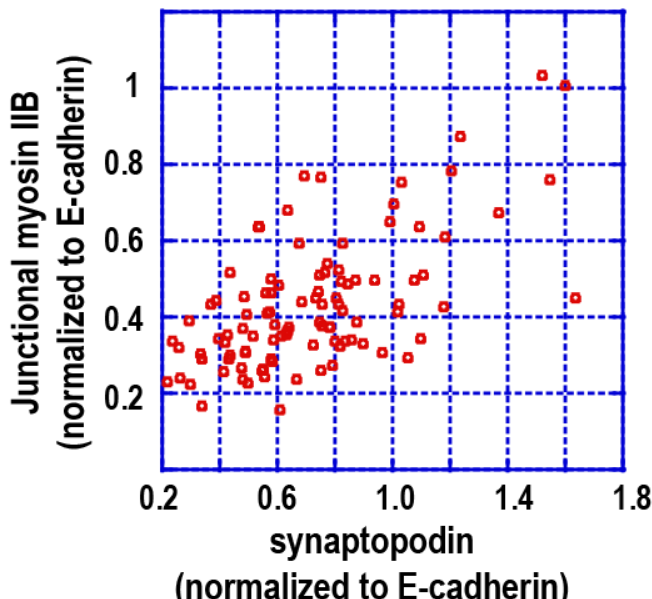

Apica Junction Apical Junction in Developing

MDCK monolayer
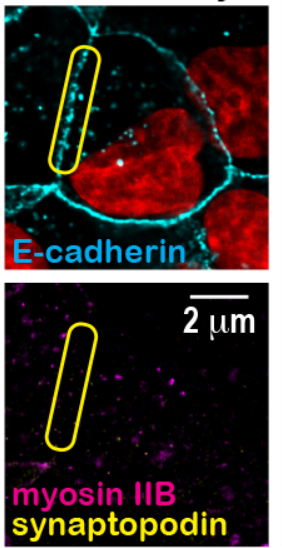

D

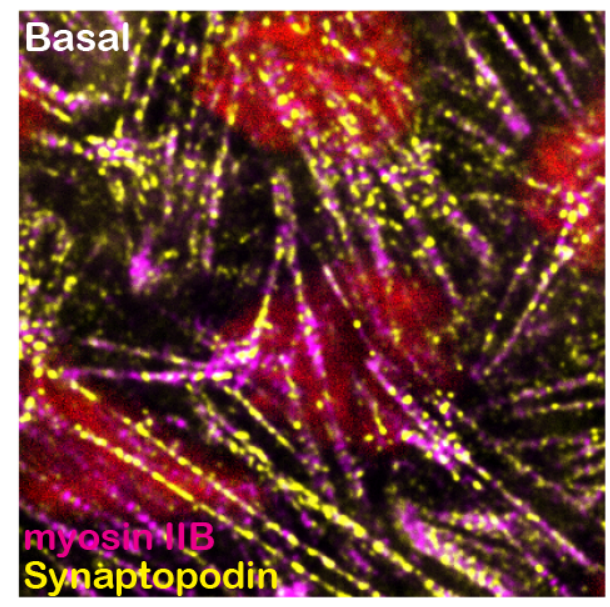

Loss of myosin IIB at apical junction in Synpo KD

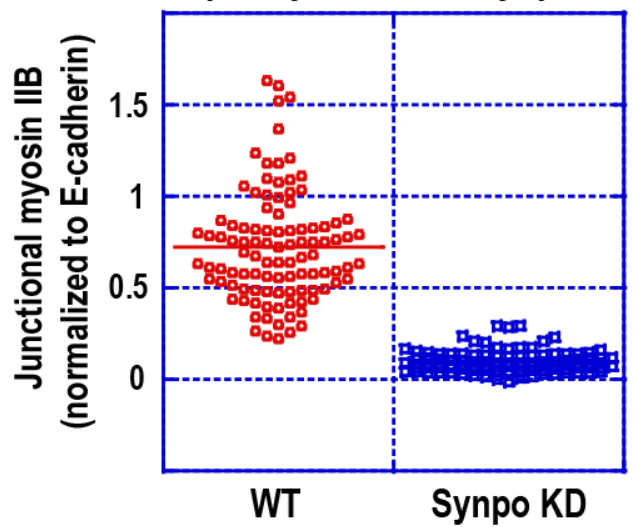

Loss of basal stress fibers in Synpo KD

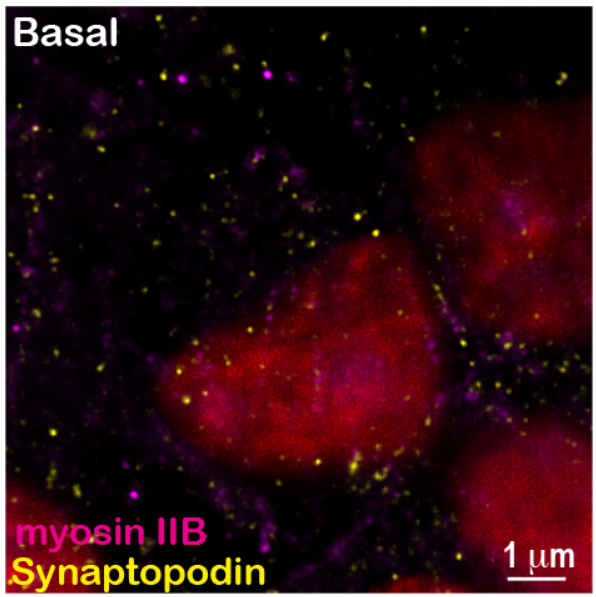

$E$

Contractomeric complexes in mature monolayer
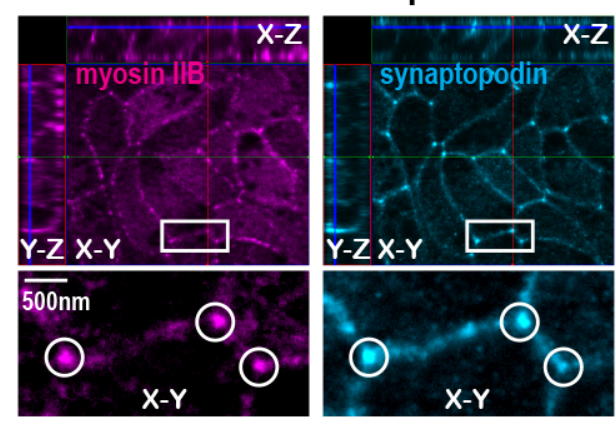

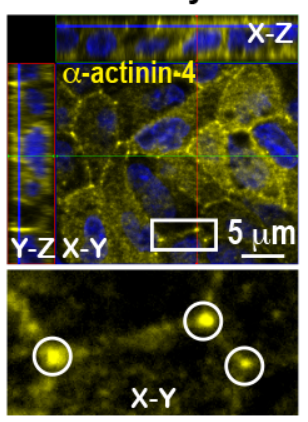

$F$

Lack of Contractomeric complex in Synaptopodin KD

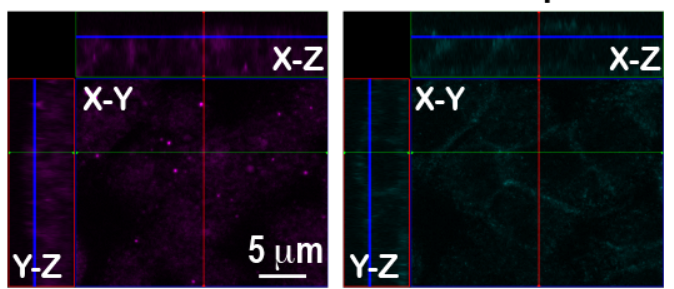

\section{(1)}

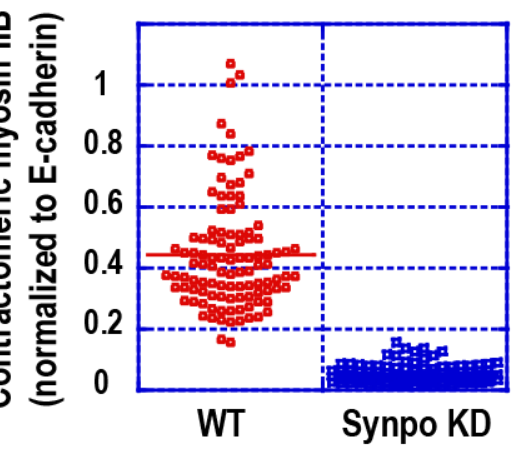

H $\frac{\text { Synpo }}{123} \frac{\text { a-cat }}{1253} \frac{\text { b-cat }}{1253} \frac{\mathrm{p} 120}{1253} \frac{\text { vinc }}{123} \frac{\text { a-act-4 }}{123} \frac{\text { MYH9 }}{123} \frac{\text { MYH10 }}{123} \frac{\text { Rho }}{123} \frac{\text { Mrip }}{123} \frac{\text { p-MLC }}{123}$

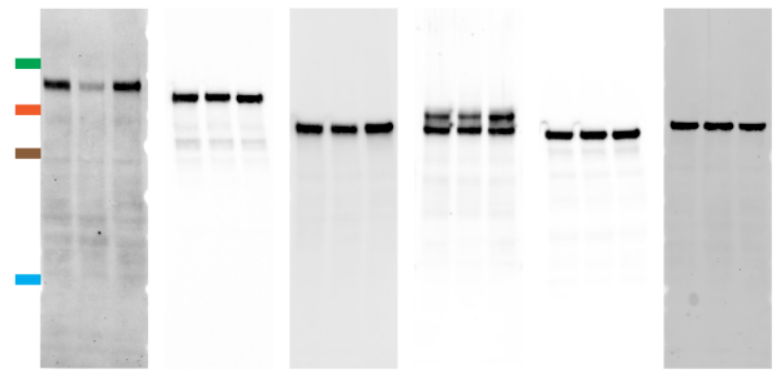

Y-Z

$\rightarrow-\infty-\infty$

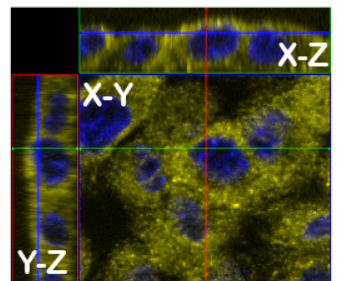


A

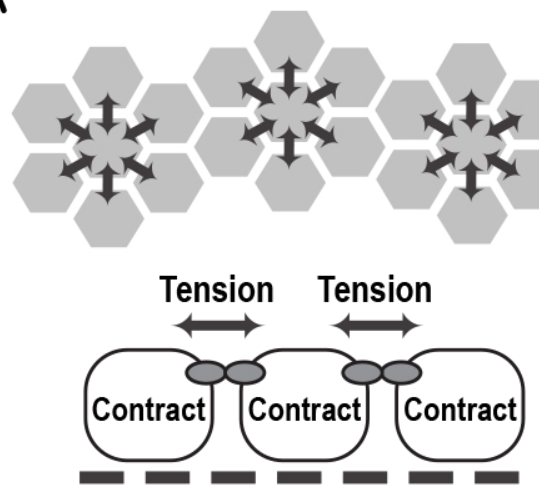

B

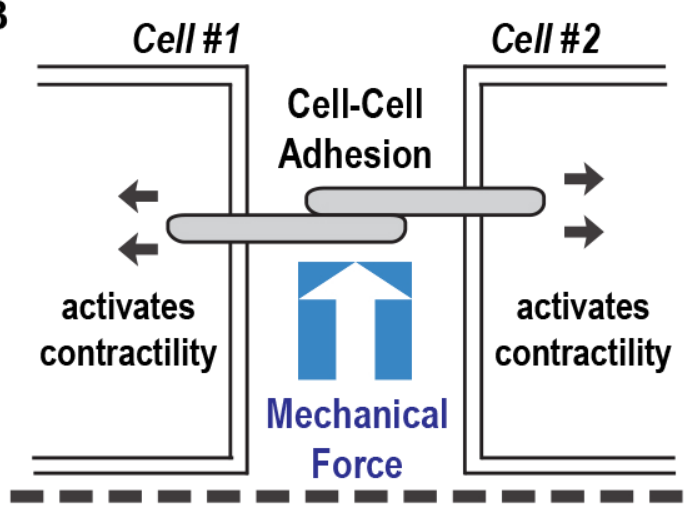

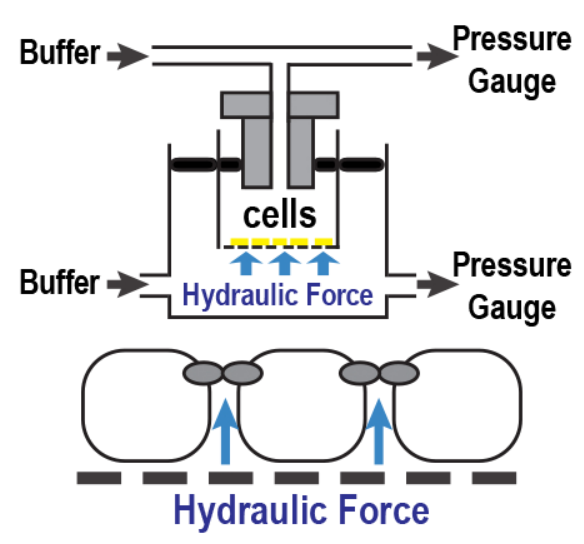

C
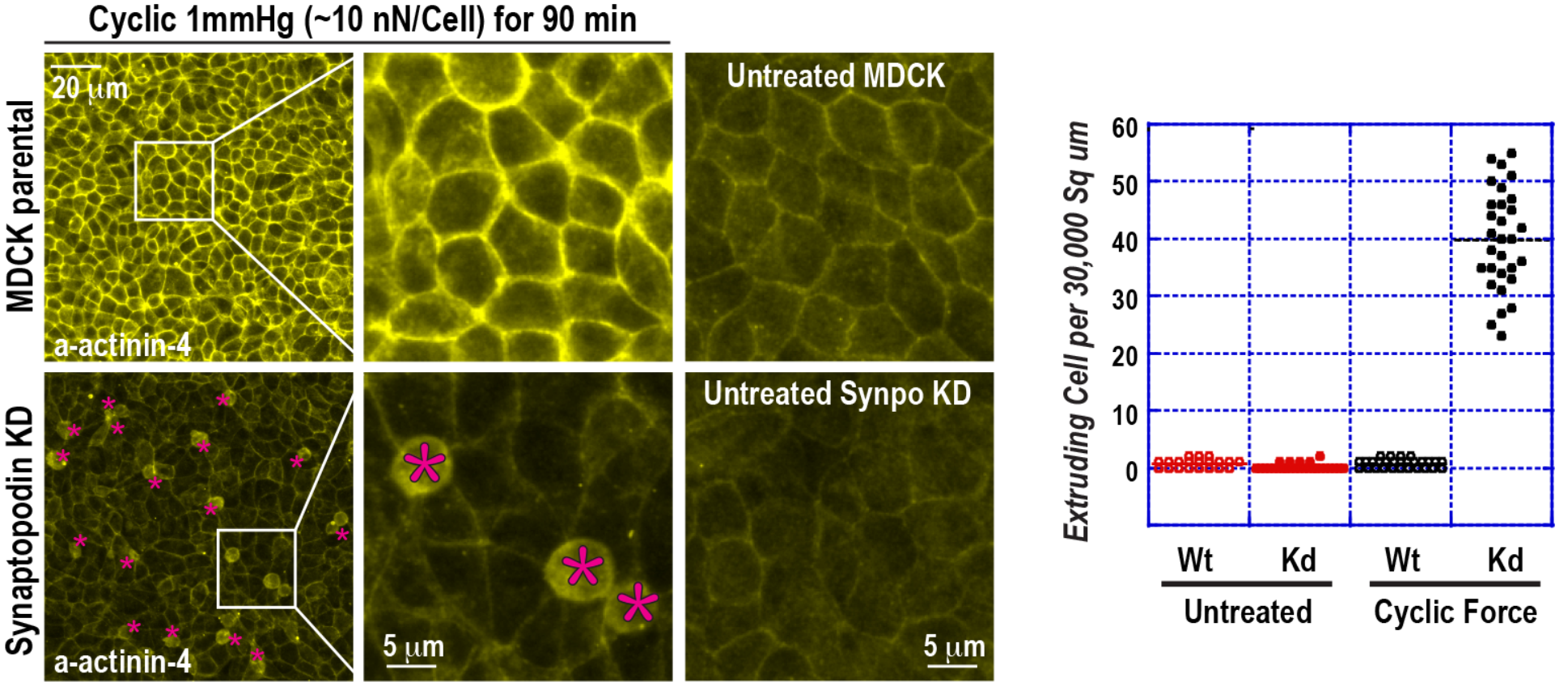

Figure 5 


\section{A}

(which was not certif by peer review) is the author/funder Immunofluorescence of Fixed cells
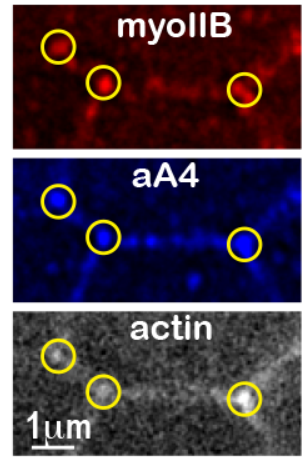

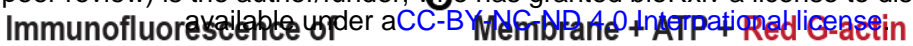
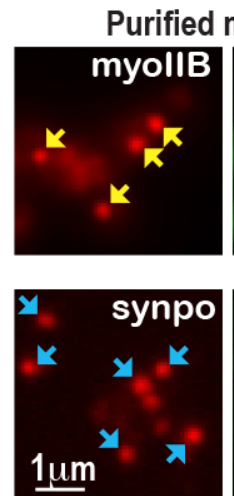
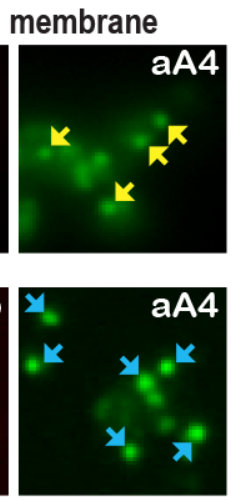
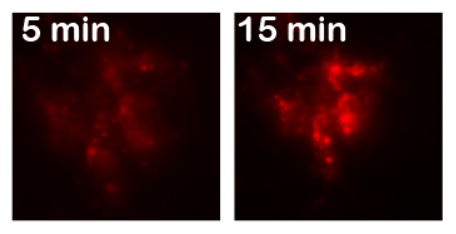

Membrane + ATP + Red G-actin + Blebbistatin

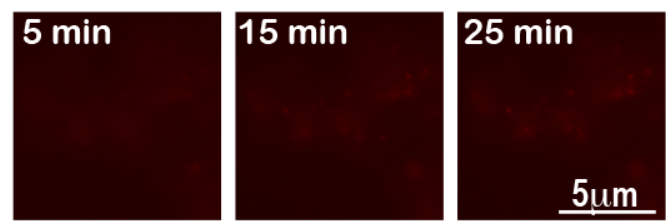

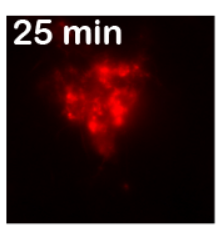
+ Green G-actin

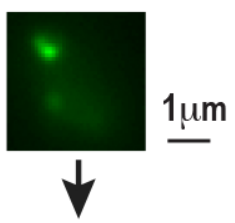

myollB (Су3) mmunofluorescence

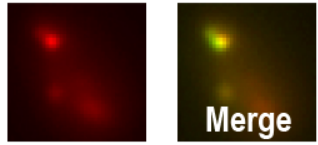

E

Junction-enriched membrane sheet $\downarrow$

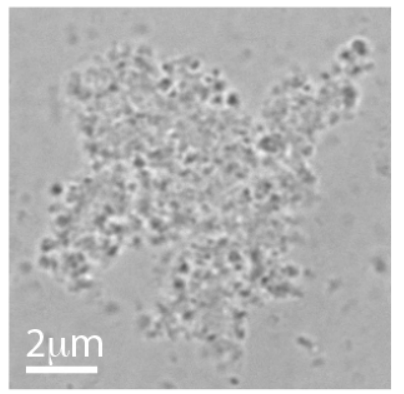

$\mathbf{F}$
Junction-enriched membrane sheet<smiles>[CH][AlH2]</smiles>

High-salt (HS) Extraction<smiles>C1C2CC12</smiles>

Purify HS-stripped membranes by spinning through $20 \%$ sucrose cushion<smiles>[CH]1[CH]C1</smiles>

Add recombinant alpha-actinin-4 proteins

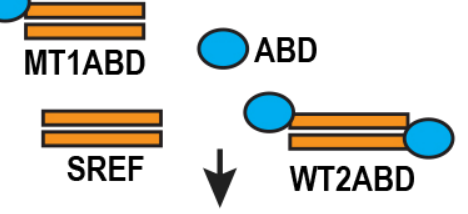

Actin Assembly Assay

+ Red or Green G-actin + ATP

Alexa 647 F-actin Bundling Assay MT1ABD $2 \overline{\mu \mathrm{m}}$ WT2ABD

\section{Alexa 647 F-actin Alexa 647 F-actin}

Recombinant alpha-actinin-4 with only 1 actin-binding domain (MT1ABD) cannot crosslink actin filaments
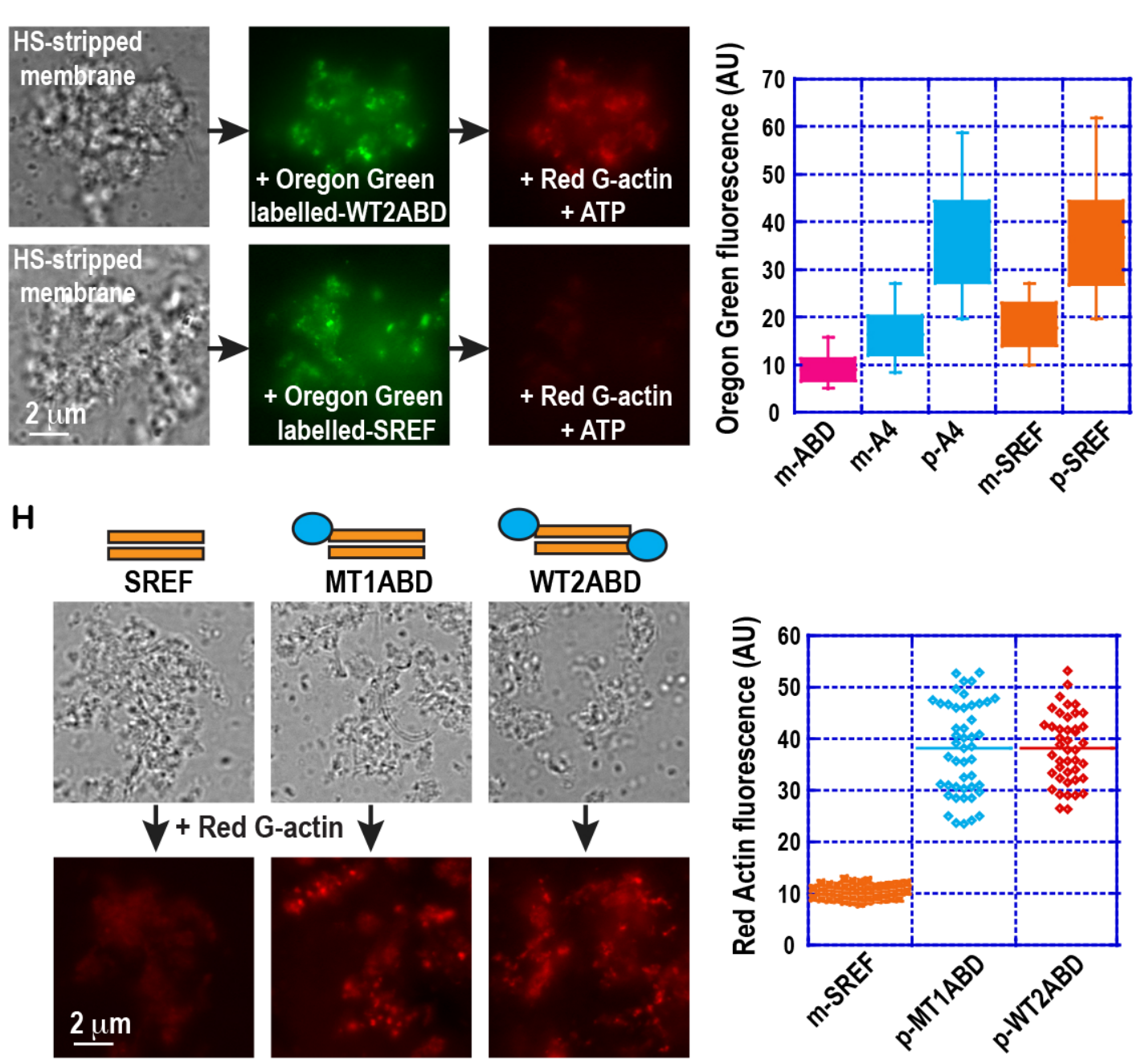

Negative-stained EM

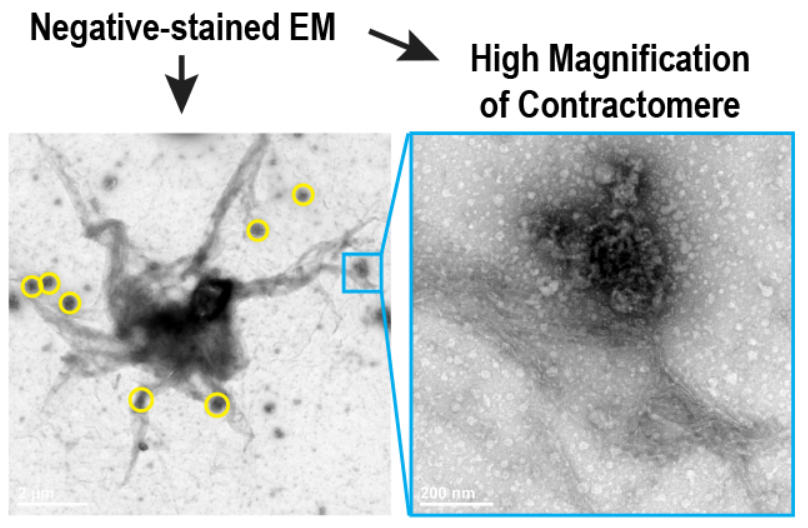

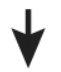

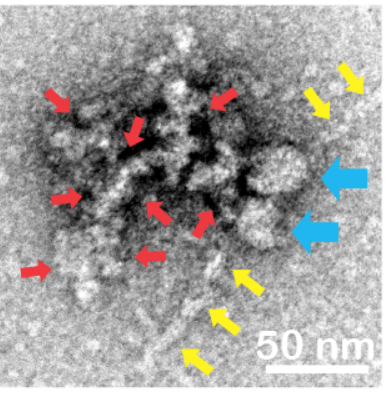

G 

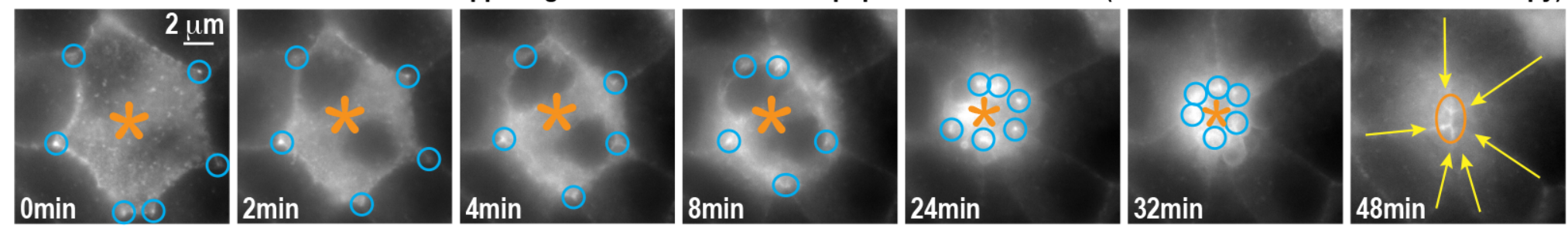

B
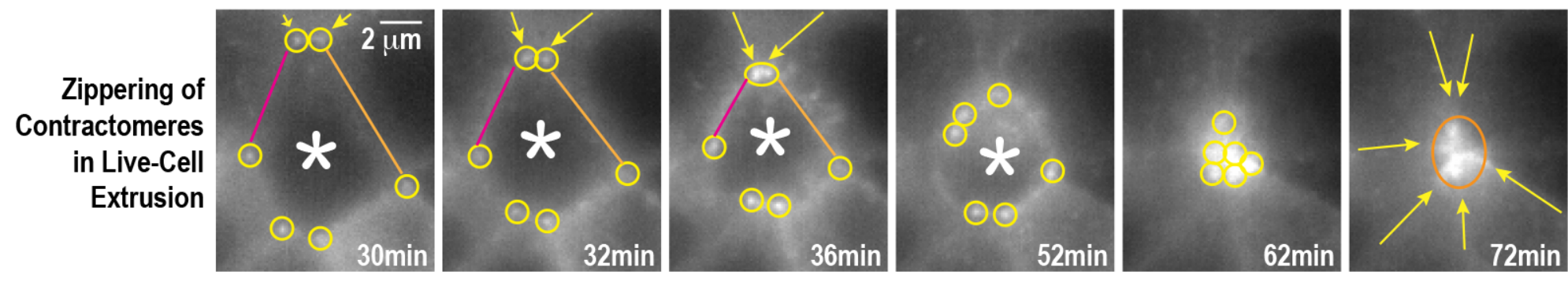

C

Structured Illumination Microscopy (SIM) -

Contractomere Zippering in Apoptotic Cell Extrusion
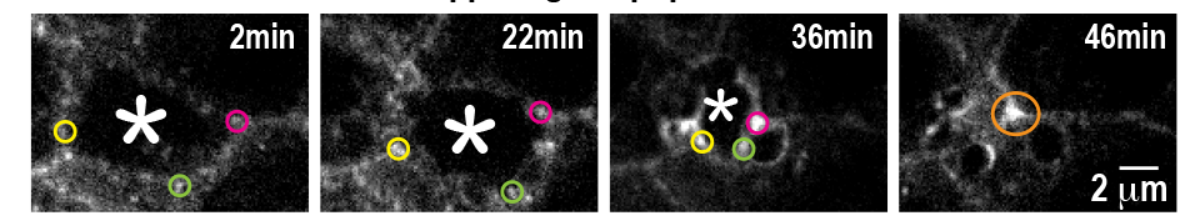

Contractomere Repositioning during Cell Extrusion (a-actinin-venus Wide-field microscopy)

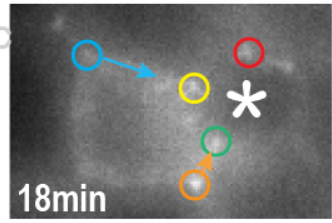

$\mathrm{F}$

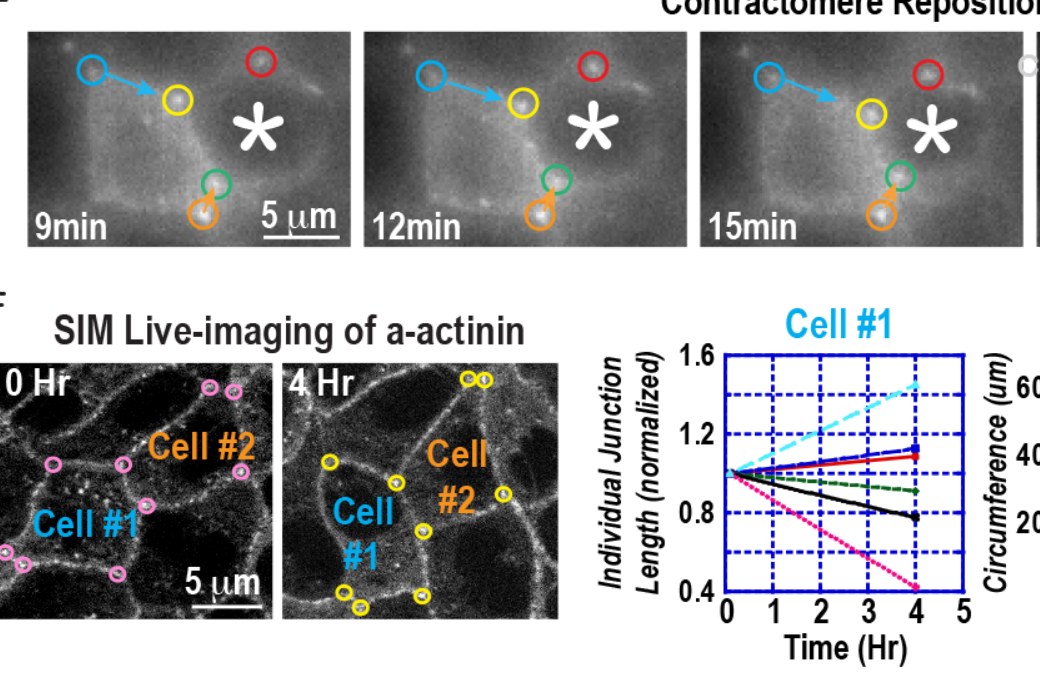

SIM Live-imaging of a-actinin
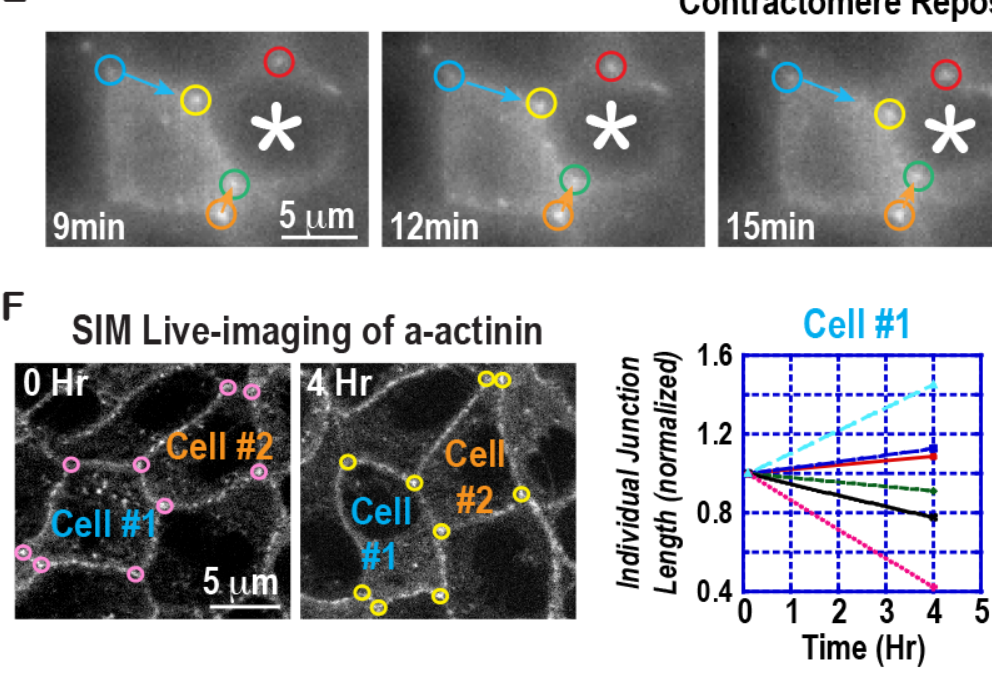

G
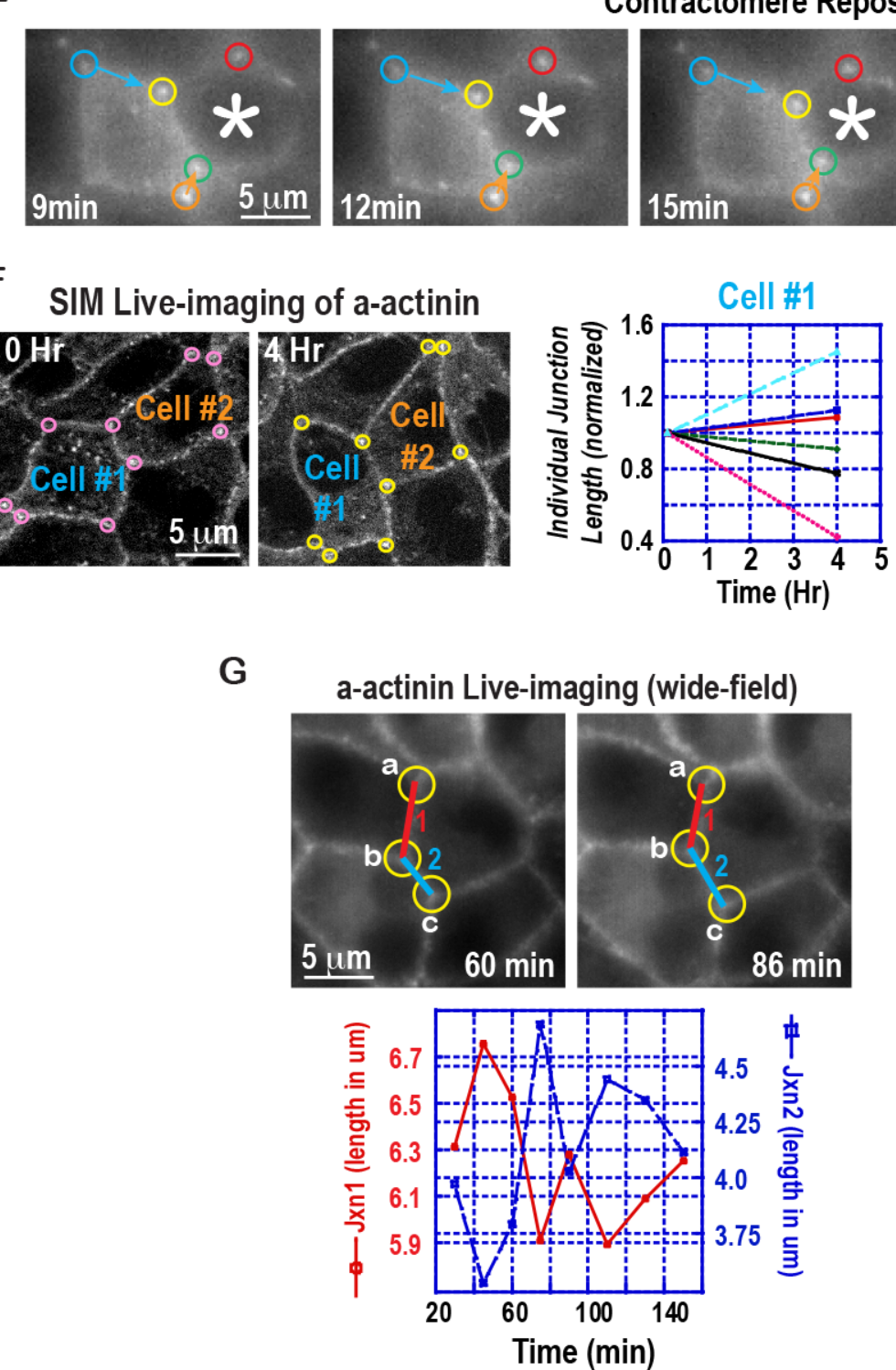

章

G

Time (min)
Cell \#1

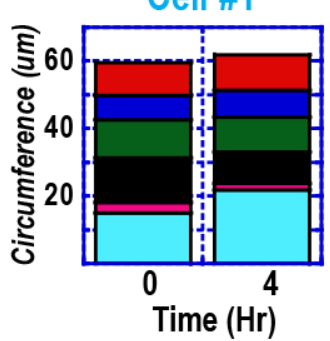

H

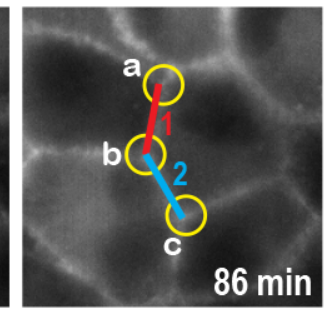

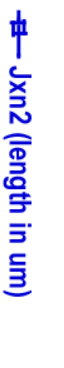
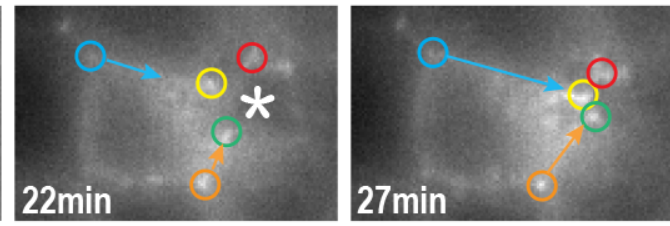

Structured Illumination Microscopy (SIM) -

Contractomere Zippering in Live-Cell Extrusion
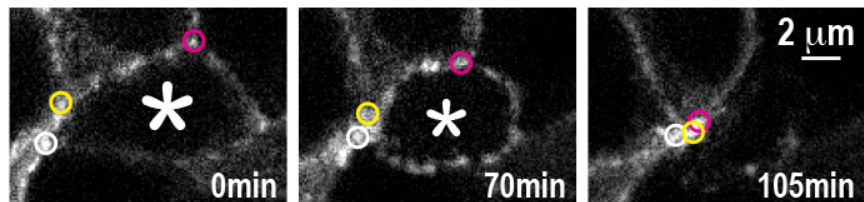

$27 \mathrm{~min}$ 
A $\alpha$-actinin anti-parallel dimer

ABD SR1 SR2 SR3 SR4 EF

a-actinin-1-sstFRET-C

(from Previous studies)

a-actinin-1-M-sstFRET (from Previous studies)

a-actinin-4-sstFRET408 (New from This Study)

a-actinin-4-sstFRET522 (New from This Study)
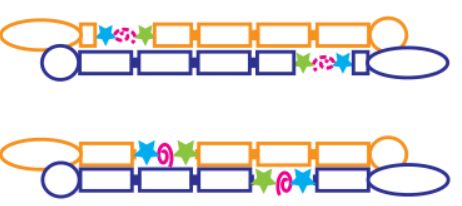

B
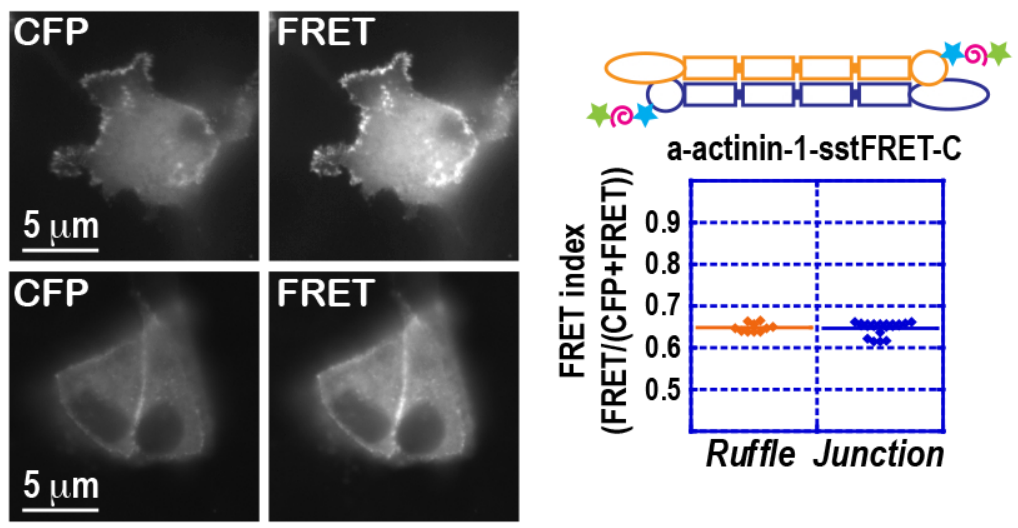

C
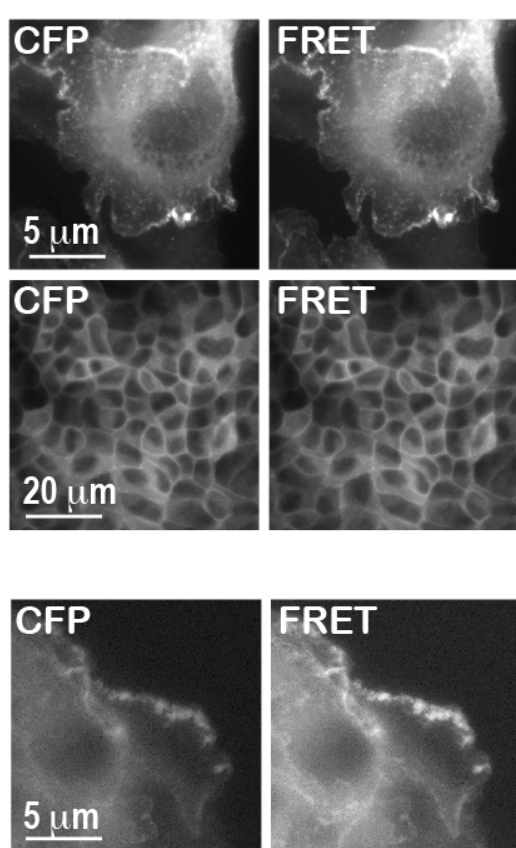

\section{CFP}

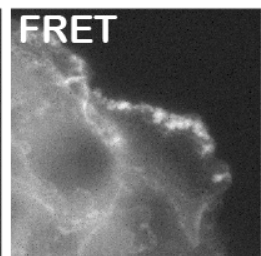

$20 \mu \mathrm{m}$
FRET index at Junction

(FRET/(CFP+FRET))

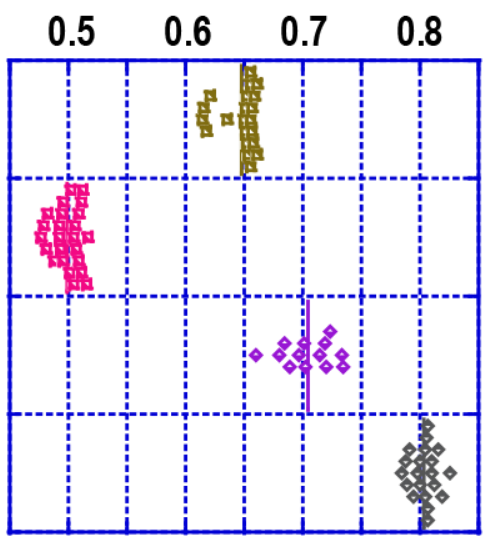

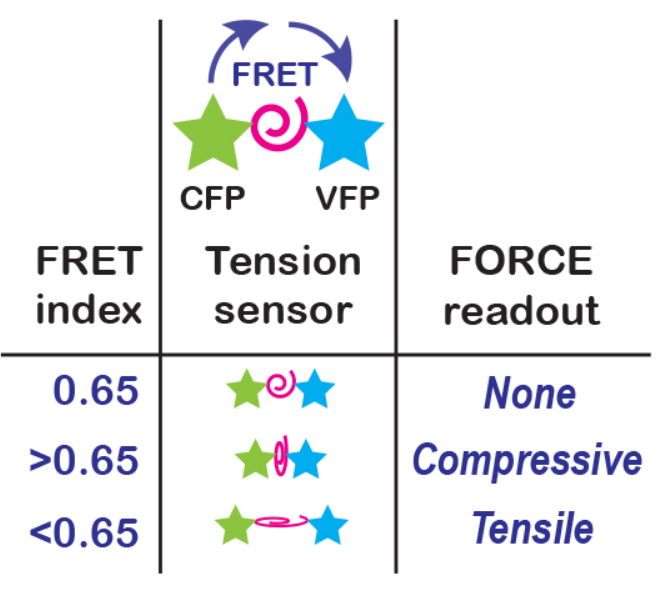

E

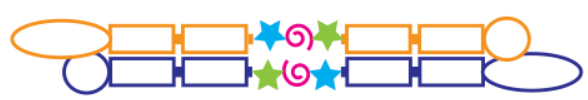

a-actinin-4-sstFRET522

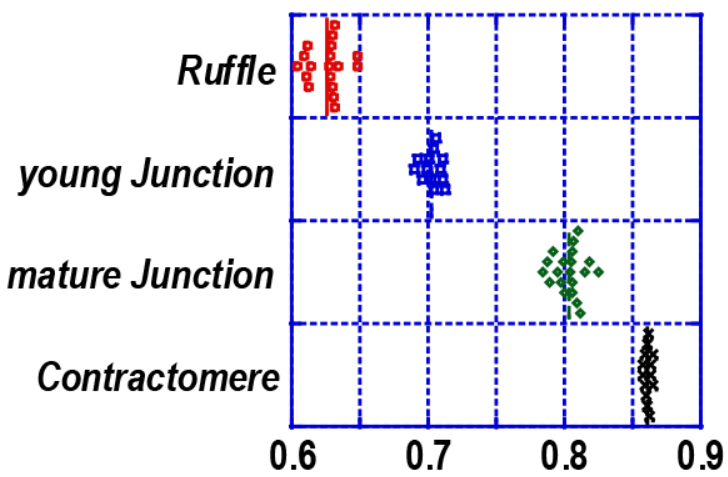

FRET index (FRET/(CFP+FRET))

F

Compressive Force exerted on junctional a-actinin-actin

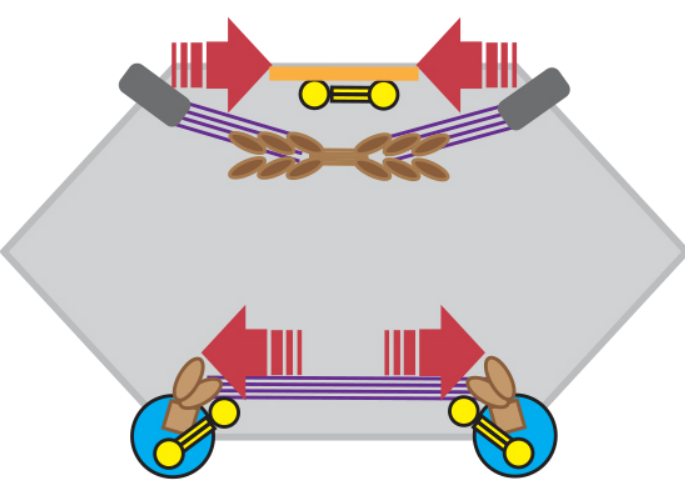

Compressive Force exerted on contractomeric a-actinin-actin 


\section{A}

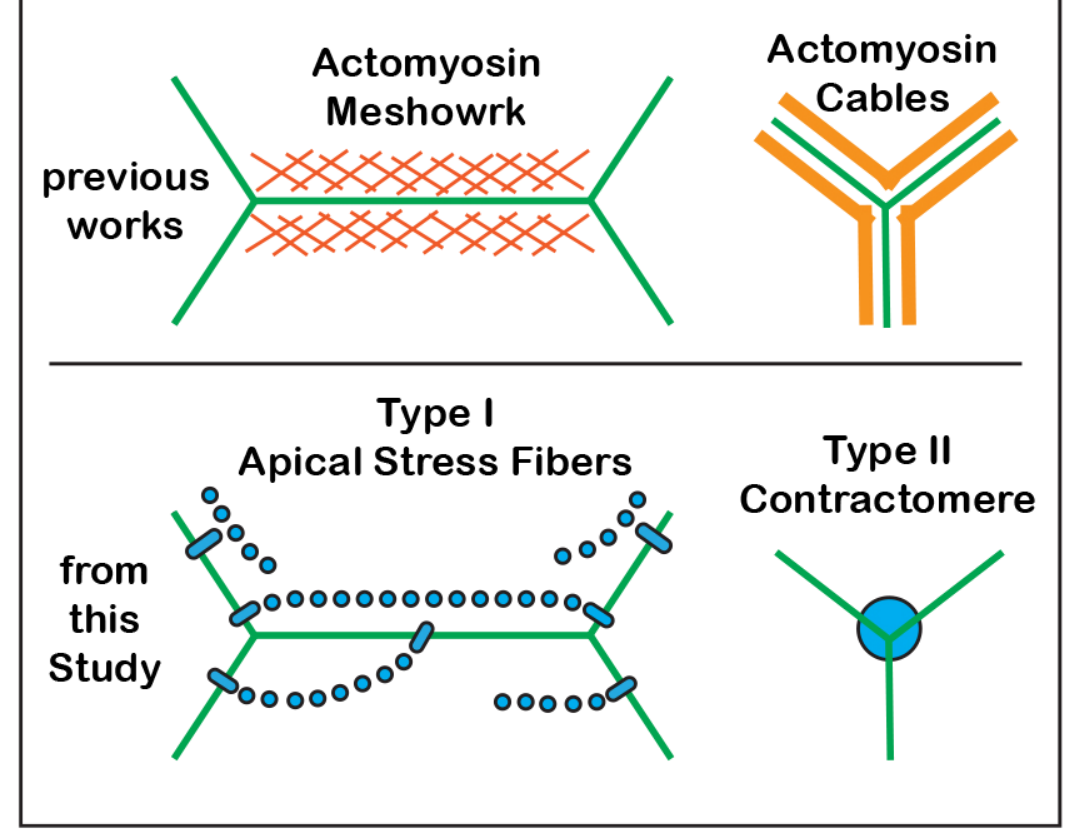

B

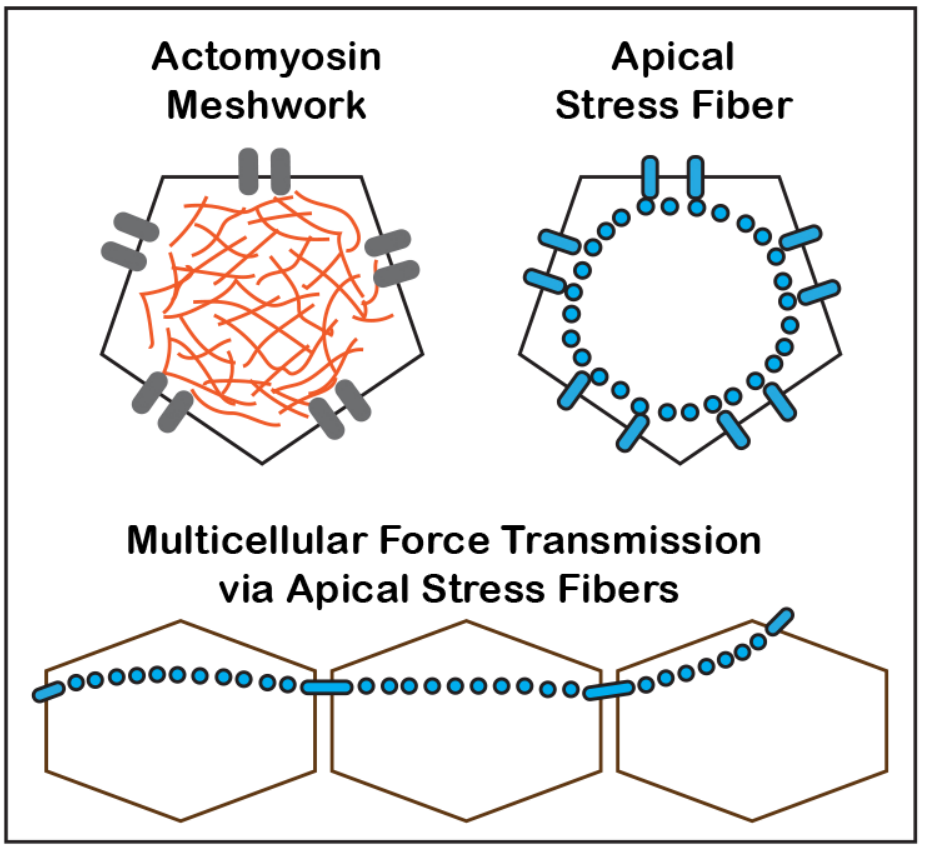

C

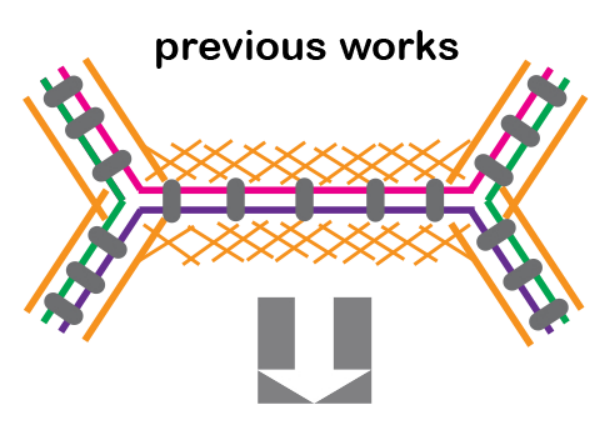

Junction Remodeling

is associated with

dissassembly of actomyosin cortex,

removel of existing junction, addition of new junction
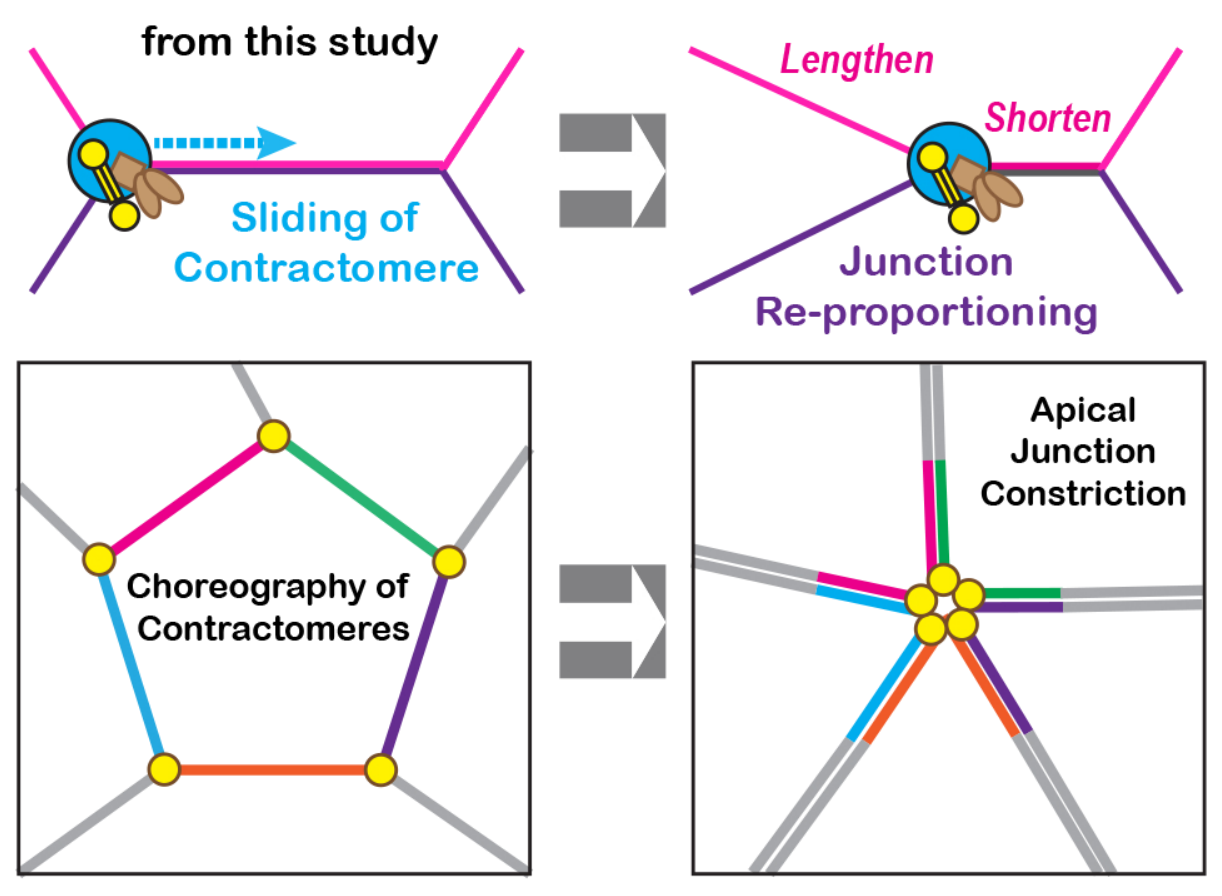

D

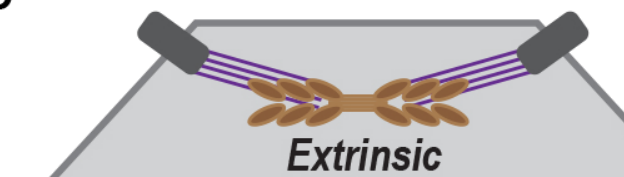

Force generated

far away from Junction

\section{Extrinsic}

Intrinsic Intrinsic

Force generated directly

by the Junction

E

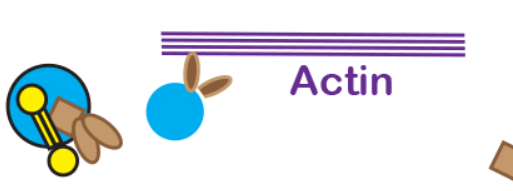

Contractomeres

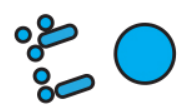

Synaptopodin Actomyosin Adhesions 


\begin{tabular}{|c|c|c|c|c|}
\hline ISOFORM & exon 1 & exon 2 & \multirow{2}{*}{ ISOFORM } & \\
\hline & \multirow{2}{*}{1245} & \multirow{2}{*}{$920 \quad 1147$} & & \\
\hline $\mathrm{X} 1$ & & & \multicolumn{2}{|c|}{ X1 $\quad$ T84, Podocyte } \\
\hline A & & & \multicolumn{2}{|c|}{ MDCK, HUVEC, Podocyte, C2bbE1 } \\
\hline B & & & \multicolumn{2}{|c|}{ Brain membranes, kidney extracts } \\
\hline C & & & C & Kidney extracts \\
\hline
\end{tabular}

(1) MDCK cells expressing isoform X1, (2) MDCK,(3) Podocyte, (4) HUVEC, (5) C2bbE1, (6) T84, (7) rat kidney, (8) MW 250, 150, 100, 75, $50 \mathrm{kD}$, (9) bovine brain membranes.

\section{$\begin{array}{lllllllll}1 & 2 & 3 & 4 & 5 & 7 & 8 & 9\end{array}$}

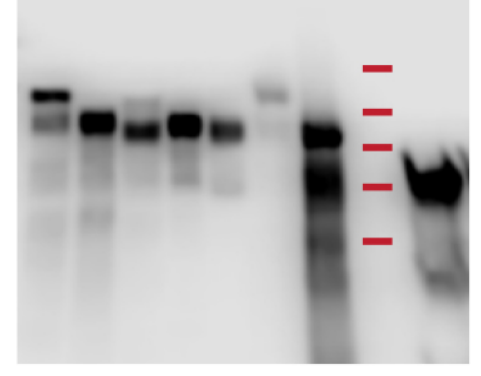




\section{Basal Stress Fibers of}

Newly-plated MDCK Monolayer

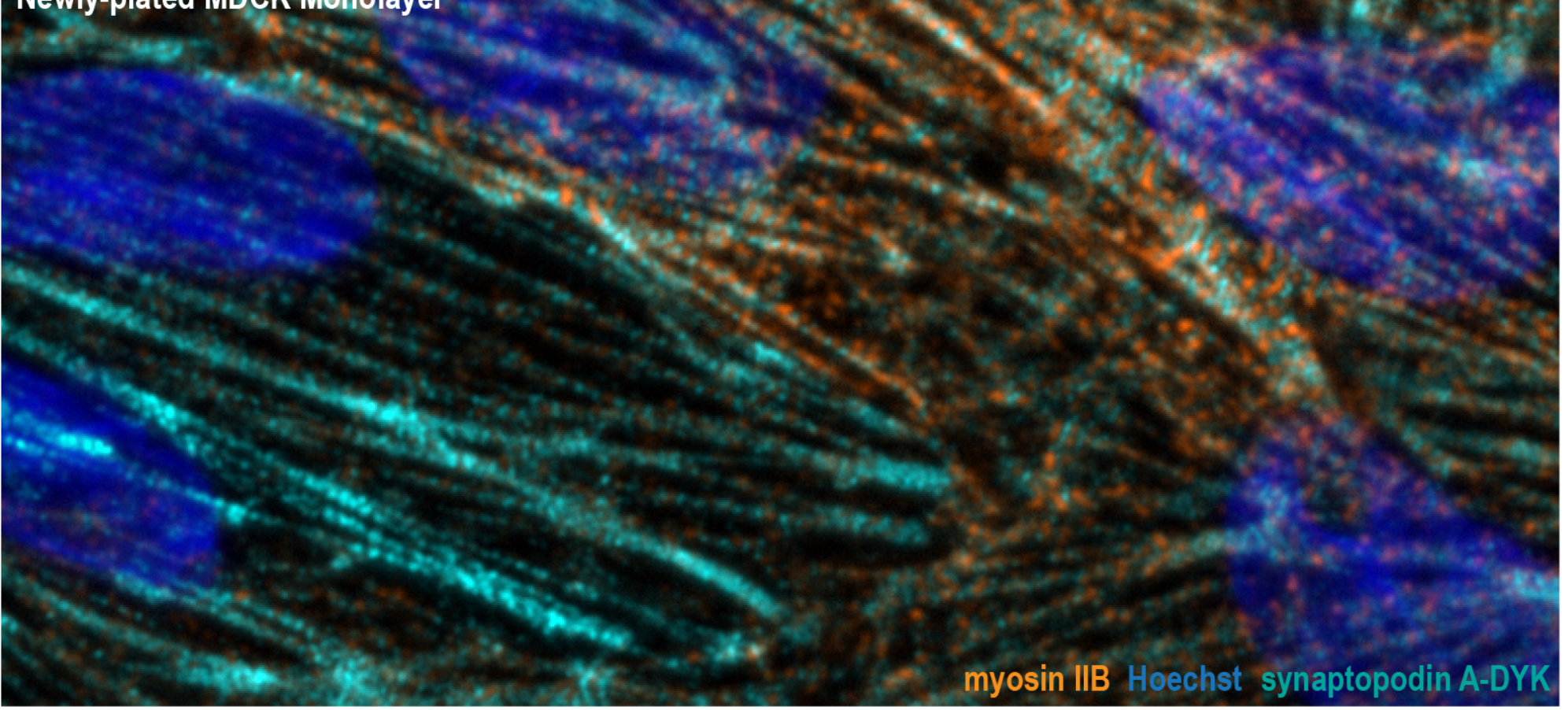

B

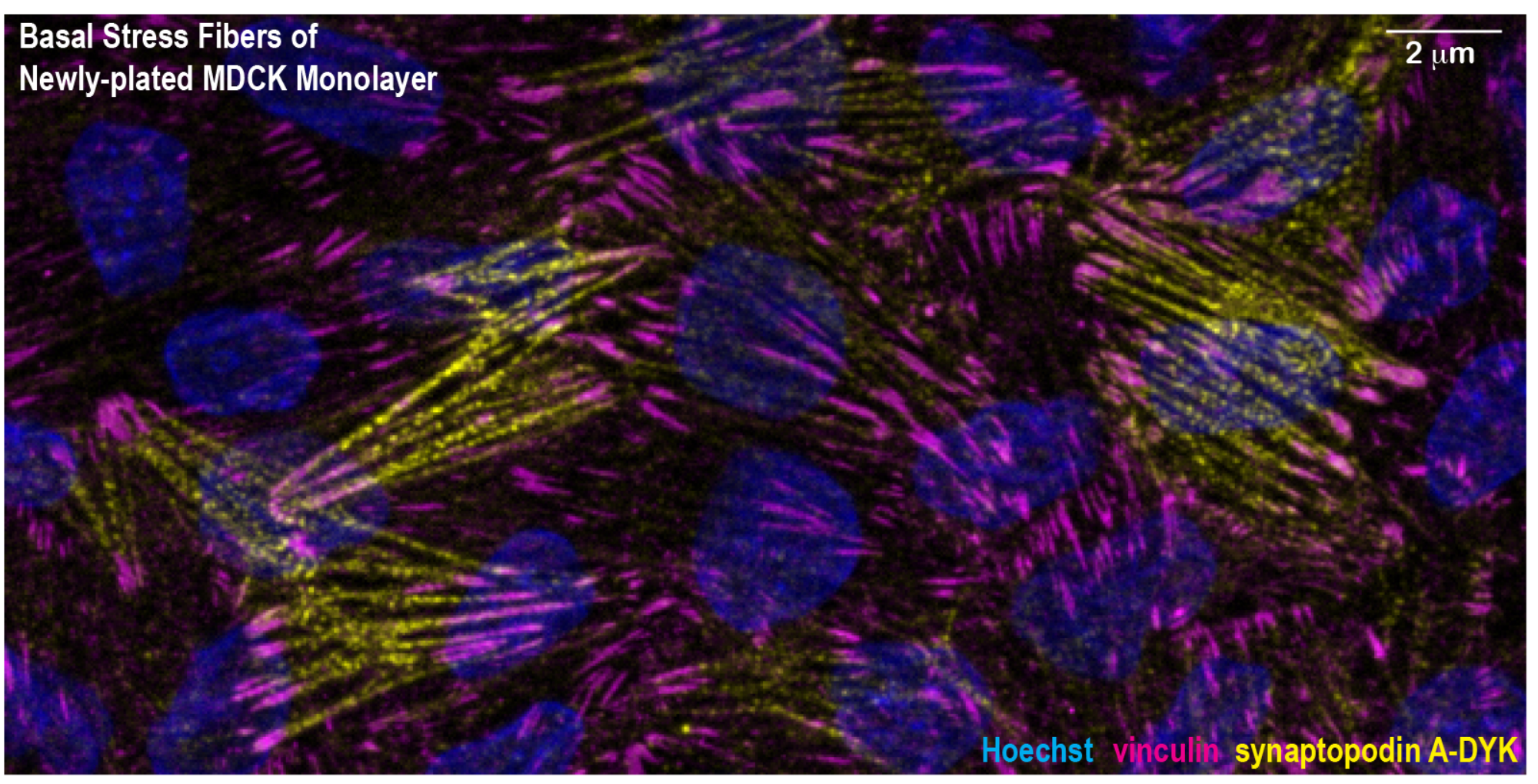

Figure S2 
A

Apical Stress fiber contains myosin IIA
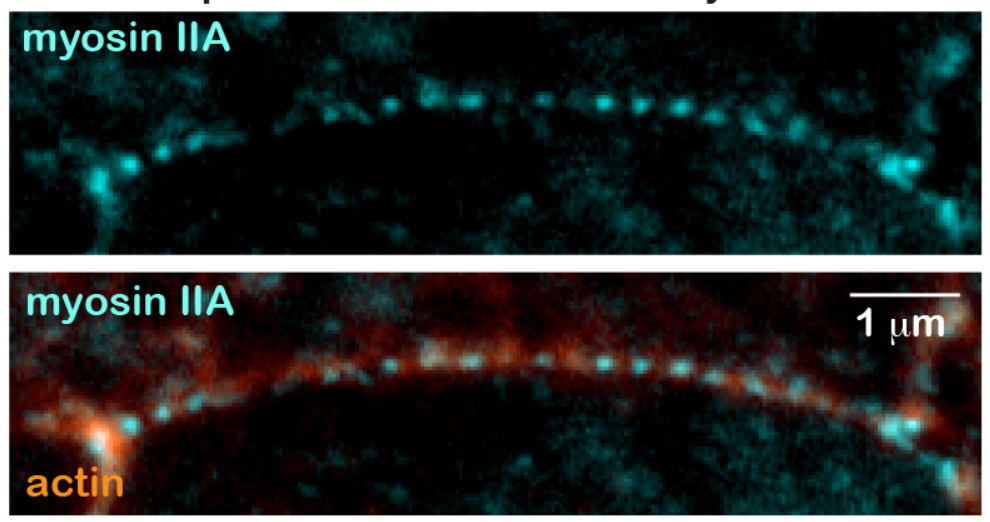

B

Basal Stress fibers contain myosin IIA
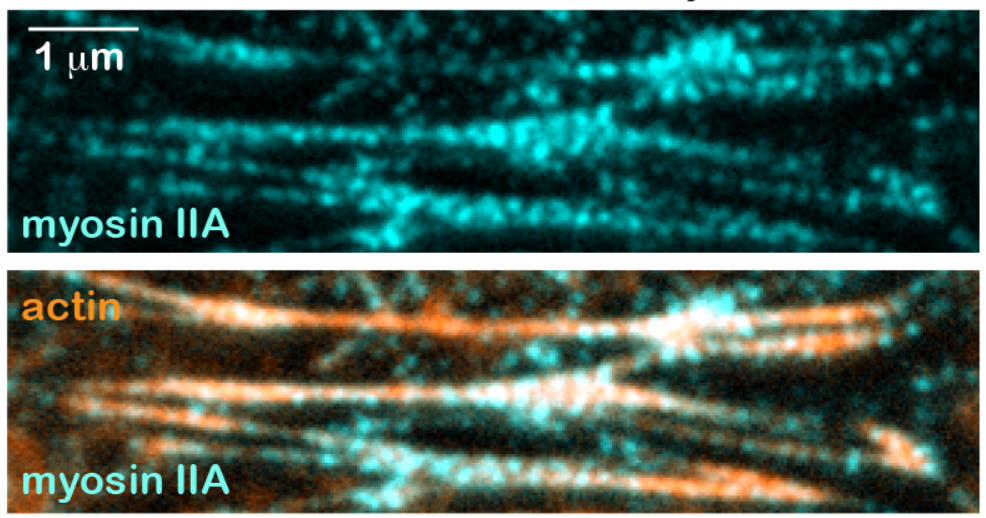

Figure S3 
MDCK parental

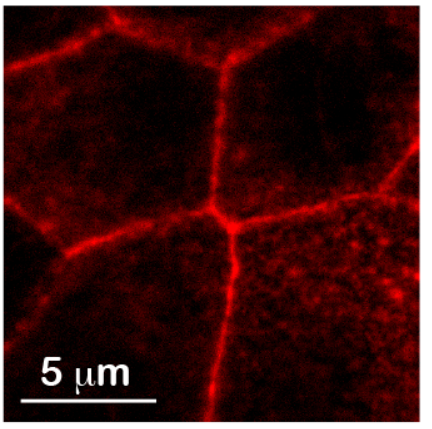

actin

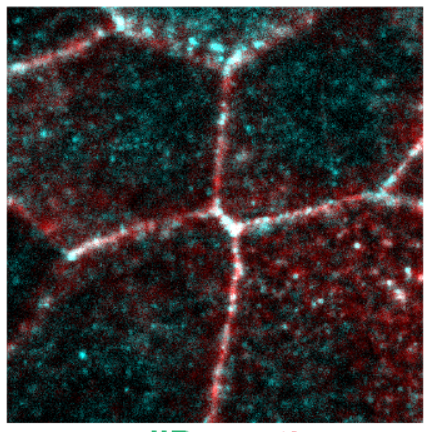

myollB actin
Synaptopodin KD

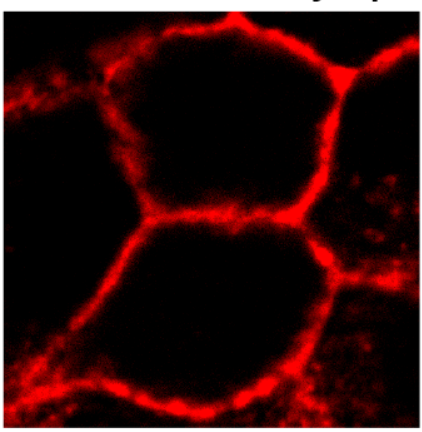

actin

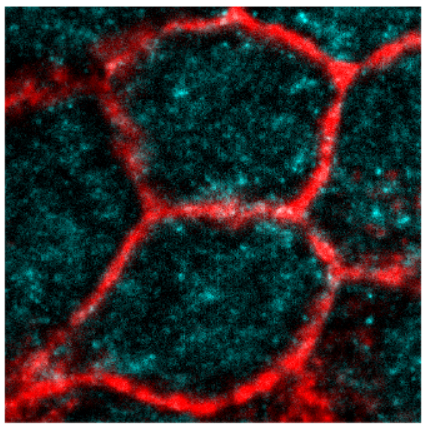

myollB actin myosin IIB is not recruited to junction in Synpo KD cells despite the presence of junctional actin

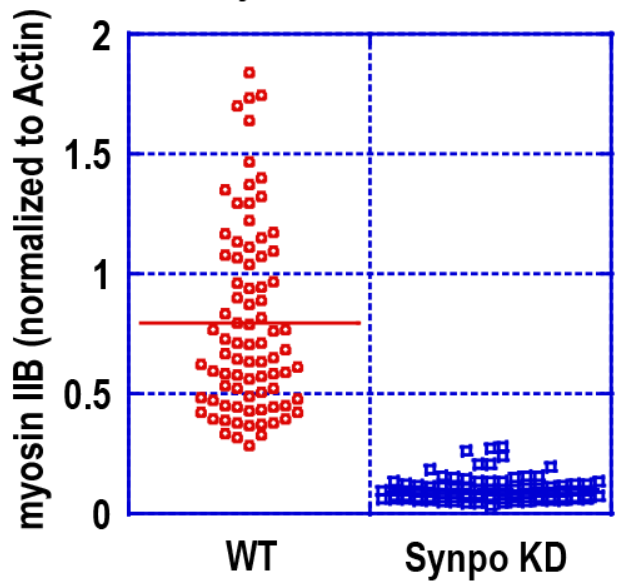


Myosin IIA remians associated with cortical Actin cytoskeleton in synaptopodin KD
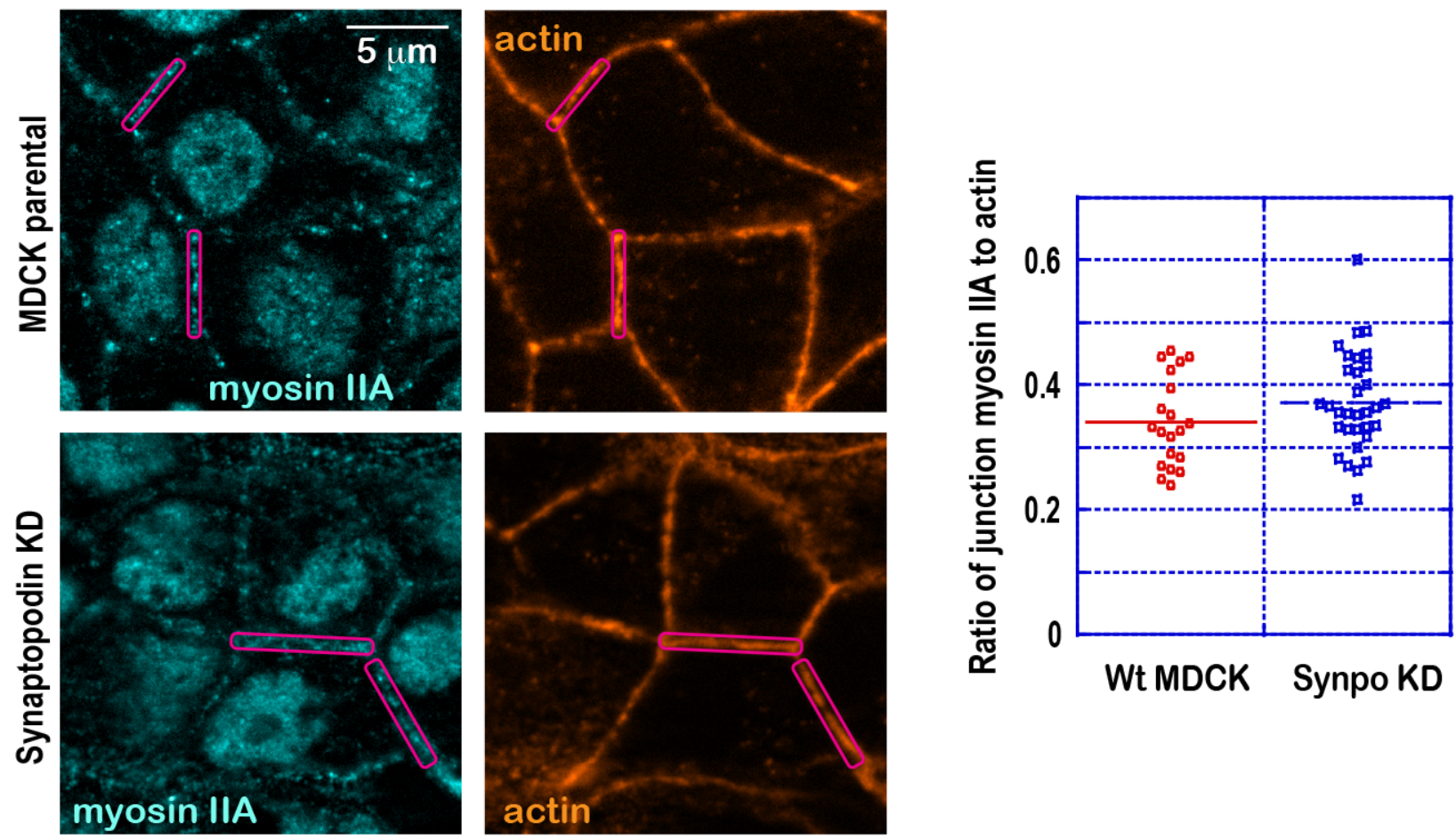

B

Synaptopodin KD converts

basal stress fibers to basal actomyosin meshwork
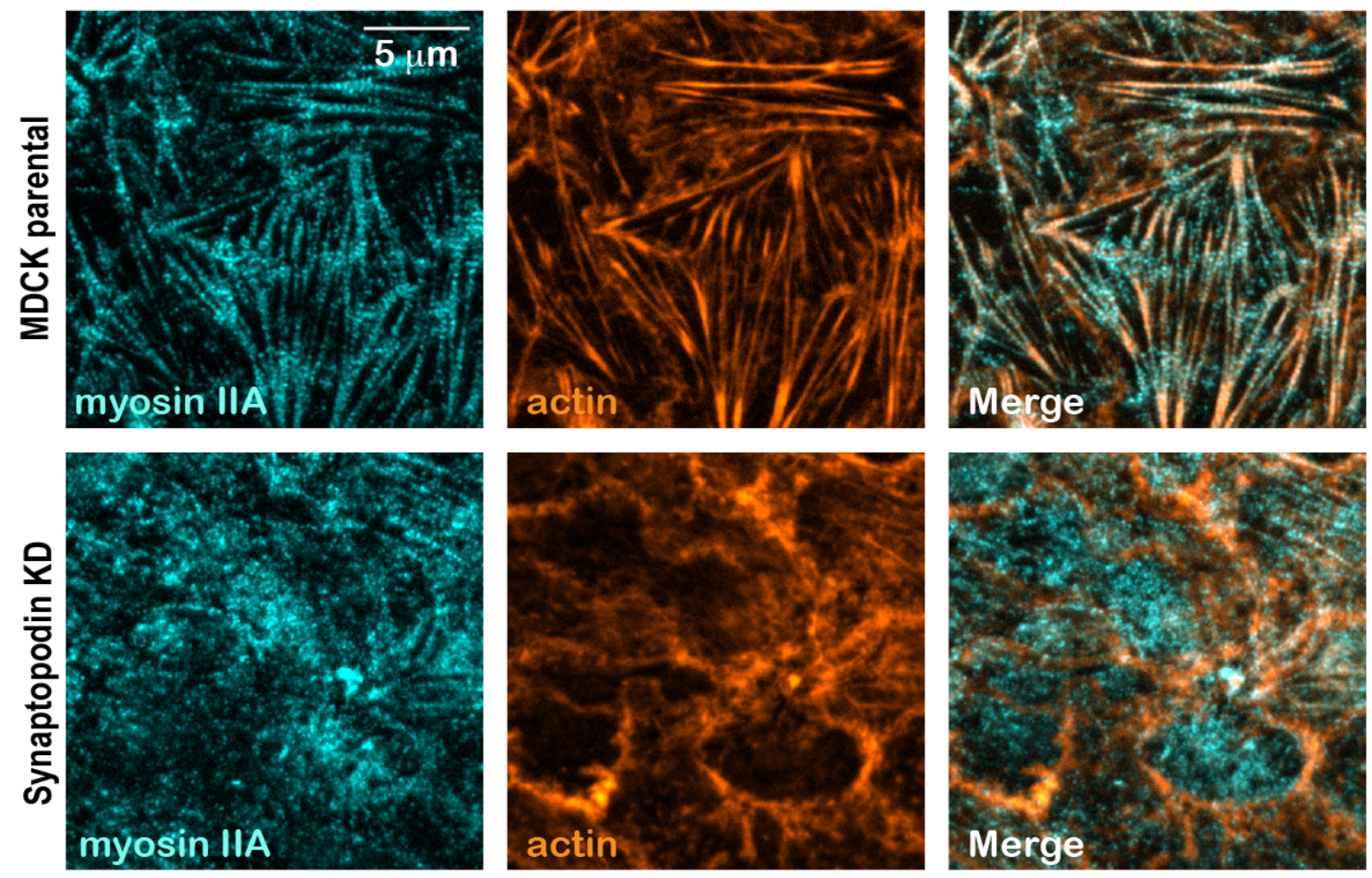

myollB (Сy3) 


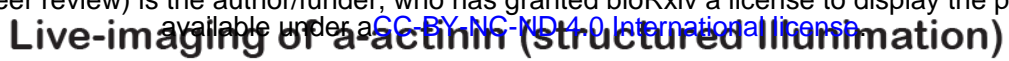
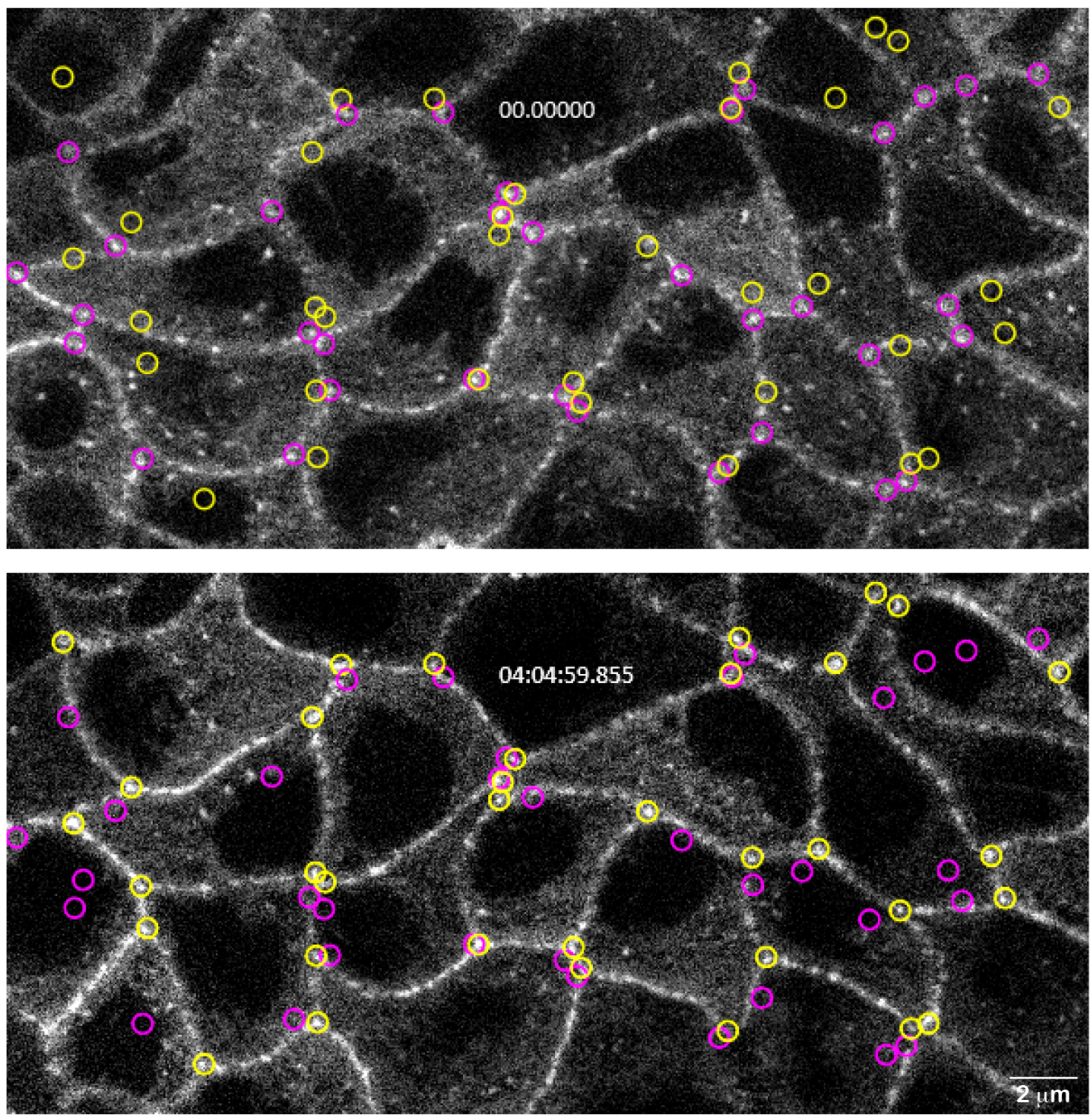

B

Live-imaging of a-actinin (wide-field)
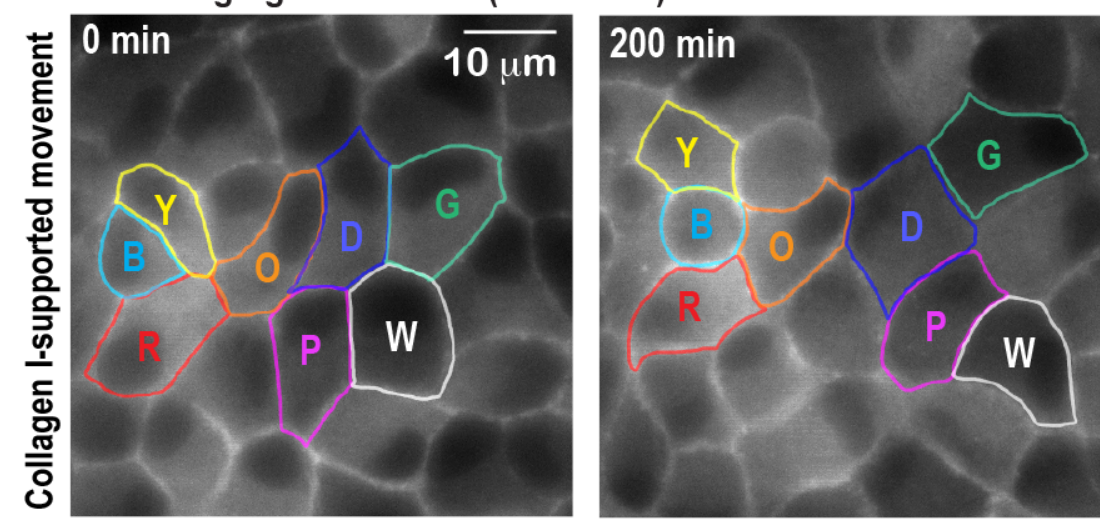

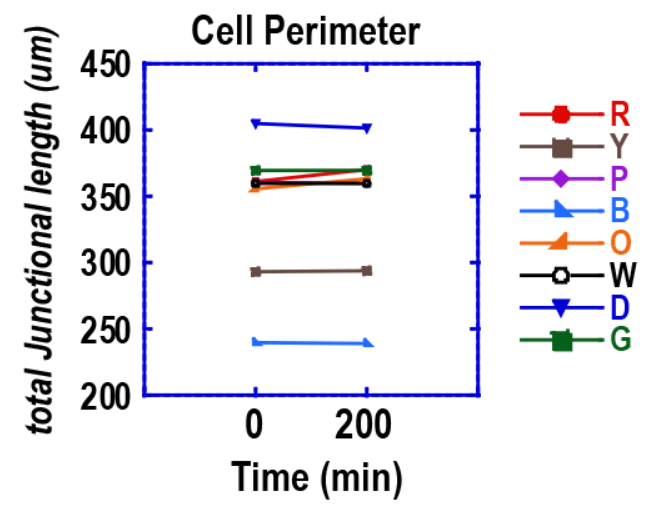

Figure S8 